\title{
Adaptive Bayesian density estimation in sup-norm
}

\author{
ZACHARIE NAULET ${ }^{1}$ \\ ${ }^{1}$ Université Paris-Saclay \\ Laboratoire de mathématiques d'Orsay \\ 91405, Orsay, France \\ E-mail: zacharie.naulet@universite-paris-saclay.fr
}

\begin{abstract}
We investigate the problem of deriving adaptive posterior rates of contraction on $\mathbb{L}_{\infty}$ balls in density estimation. Although it is known that log-density priors can achieve optimal rates when the true density is sufficiently smooth, adaptive rates were still to be proven. Here we establish that the so-called spike-and-slab prior can achieve adaptive and optimal posterior contraction rates. Along the way, we prove a generic $\mathbb{L}_{\infty}$ contraction result for log-density priors with independent wavelet coefficients. Interestingly, our approach is different from previous works on $\mathbb{L}_{\infty}$ contraction and is reminiscent of the classical test-based approach used in Bayesian nonparametrics. Moreover, we require no lower bound on the smoothness of the true density, albeit the rates are deteriorated by an extra $\log (n)$ factor in the case of low smoothness.
\end{abstract}

MSC2020 subject classifications: Primary 62G07; secondary 62G20

Keywords: Bayesian density estimation; supremum norm; adaptation

\section{Introduction}

We consider the problem of estimating a density $p$ with respect to Lebesgue measure on $[0,1]$ given $n$ independent and identically distributed samples $\mathbf{X}_{n}:=\left(X_{1}, \ldots, X_{n}\right)$ from the corresponding distribution $P$. We adopt the Bayesian paradigm and put a joint distribution on the log-density and the observations.

Over the decades, there has been a growing interest for the understanding of the frequentist behaviour of posterior distributions initiated by the seminal papers of Schwartz (1965); Barron, Schervish and Wasserman (1999); Ghosal, Ghosh and van der Vaart (2000). In particular Ghosal, Ghosh and van der Vaart (2000) states generic sufficient conditions for obtaining rates of concentration of the posterior distribution near the true model in some distance. The approach relies on the well-known existence of exponentially powerful test functions. The existence of such tests depends on the distance considered, and is guaranteed for the $\mathbb{L}_{1}$ or Hellinger distance between densities, and also for the $\mathbb{L}_{2}$ metric under supplementary assumptions. It is, however, now well understood that the test approach fails to give optimal rates when the risk is measured with respect to the $\mathbb{L}_{\infty}$ distance, see Castillo (2014); Hoffmann, Rousseau and Schmidt-Hieber (2015); Yoo, Rousseau and Rivoirard (2017). 
The failure of the classical approach for $\mathbb{L}_{\infty}$ rates is unfortunate because one has in general a better intuition of the shape of $\mathbb{L}_{\infty}$ balls rather than Hellinger balls, making the $\mathbb{L}_{\infty}$ risk a more natural distance for evaluating performance of estimators. From a frequentist point of view, density estimation in sup-norm is now well understood. Minimax lower bounds can be found in Hasminskii (1979) while upper bounds can be found for instance in Ibragimov and Hasminskii (1980); Goldenshluger and Lepski (2014).

For Bayesian procedures, concentration on $\mathbb{L}_{\infty}$ balls is much less understood. For the non-adaptive case, the first result goes back to Giné and Nickl (2011) where optimal rates are obtained in white-noise regression using conjugacy arguments. In the same paper, the authors obtained (possibly adaptive) rates for density estimation in sup-norm using a testing approach, but failed to achieve optimality. Using conjugacy arguments, Yoo and Ghosal (2016) also obtain non-adaptive but optimal rates for estimating a regression function. Scricciolo (2014) adapts the techniques of Giné and Nickl (2011) to obtain optimal rates when the true density is analytic. The first non-adaptive optimal result in density estimation for non ultra-smooth densities is to be credited to Castillo (2014), where the author uses techniques based on semi-parametric Bernstein-von Mises theorems. His approach, however, requires a minimal smoothness to be applicable. Recently, Castillo (2017) obtained non-adaptive but optimal rates for density estimation in sup-norm using Pólya trees prior, with no lower bound required on the smoothness.

The existence of adaptive and optimal results is, to our knowledge, even more limited. The first successful result is in Hoffmann, Rousseau and Schmidt-Hieber (2015) where the authors get adaptive optimal rates in $\mathbb{L}_{\infty}$ norm for white-noise regression using a spike-and-slab prior. They also obtain adaptive and optimal rates in density estimation, though their result is rather an existence result as their abstract sieve prior is not computable. More recently, Yoo, Rousseau and Rivoirard (2017) obtained adaptive optimal rates in $\mathbb{L}_{\infty}$ norm for estimating a regression function, using a white-noise approximation of the likelihood to adapt the techniques developed in Hoffmann, Rousseau and Schmidt-Hieber (2015). Since the first version of the current paper, Castillo and Mismer (2019) have introduced spikeand-slab Pólya trees and built upon the results of Castillo (2017) to obtain adaptive contraction rates, though the arguments we use are different.

In density estimation, it is not obvious to proceed as Yoo, Rousseau and Rivoirard (2017) and reduce the problem to white-noise regression, although it is known those models are equivalent (in the Le Cam sense) under certain assumptions. Here, instead, we propose a different approach. We obtain in Section 3 a general contraction result for log-density priors with independent wavelet coefficients. This result is the building block of our main Theorem 2.2 about the spike-and-slab log-density prior. The posterior spike-and-slab is known to be the Bayesian analogue of hard thresholding (Härdle et al., 2000), as already noticed by Hoffmann, Rousseau and Schmidt-Hieber (2015); Yoo, Rousseau and Rivoirard (2017). As such, it constitutes the prototypical example of model for which we expect adaptive and optimal $\mathbb{L}_{\infty}$ contraction. Unlike Hoffmann, Rousseau and Schmidt-Hieber (2015); Yoo, Rousseau and Rivoirard (2017), however, the present paper does not exploit the thresholding property of the spike-and-slab posterior to establish the posterior support of the wavelet coefficients, but uses a more classical approach.

In the case of the spike-and-slab prior, we show that our method can be applied to obtain minimax optimal and adaptive posterior contraction. More precisely, we show that if $L:=\log p \in B_{\infty, \infty}^{s}[0,1]$, where $B_{\infty, \infty}^{s}[0,1]$ denotes the Hölder-Zygmund space with smoothness $s>1 / 2$ (see Definition 2.1), then there exists $M>0$ such that as $n \rightarrow \infty$,

$$
\mathbb{E}_{L} \Pi\left(L^{\prime}:\left\|L^{\prime}-L\right\|_{\infty} \leq M \varepsilon_{n}^{*}(s) \mid \mathbf{X}_{n}\right)=1+o(1),
$$


where $\varepsilon_{n}^{*}(s)$ is the minimax rate over bounded balls in $B_{\infty, \infty}^{s}[0,1]$ under $\mathbb{L}_{\infty}$ loss (Donoho et al., 1996)

$$
\varepsilon_{n}^{*}(s):=\left(\frac{\log n}{n}\right)^{\frac{s}{2 s+1}} .
$$

Interestingly, our method can be applied to obtain adaptive rates in the region $0<s \leq 1 / 2$, which to the best of our knowledge is the first result of this type in the Bayesian literature for methods not relying on conjugacy arguments. The rates we obtain in this region are, however, slightly deteriorated by a factor $\log (n)$.

In contrast with previous results in $\mathbb{L}_{\infty}$ loss, the approach used in this paper is somewhat less specific and uses the same kind of arguments as for the master theorem of Bayesian nonparametrics (Ghosal, Ghosh and van der Vaart, 2000; Ghosal and van der Vaart, 2007a,b). In particular, it relies on the existence of suitable test functions and proving the prior positivity of some neighborhoods, in apparent contradiction with the folk wisdom that no test for the $\mathbb{L}_{\infty}$ loss has enough power to obtain optimal rates Hoffmann, Rousseau and Schmidt-Hieber (2015); Yoo, Rousseau and Rivoirard (2017). This contradiction is only apparent, as here we require to test only very specific kind of alternatives, and exponentially consistent tests are not needed. Although hard to generalize, we believe the present paper shows that the traditional approach of (Ghosal, Ghosh and van der Vaart, 2000; Ghosal and van der Vaart, 2007a,b) is more powerful than we believed, giving hope for the existence of general contraction results in strong norms.

This article comes with a supplementary material, which contain additional proofs and various classical results about the spike-and-slab log-density prior. We adopt the convention that every section, subsection, theorem, etc. of the supplemental has label prefixed by $\mathrm{S}$ and is cited in cyan. References to the main document are cited in blue with no prefix.

\section{Exponentiated random wavelet series}

\subsection{Log-density priors}

We use the $S$-regular, orthogonal, boundary corrected wavelets of Cohen, Daubechies and Vial (1993), referred to as the CDV basis. We denote this basis by $\left\{\varphi_{j, k}:(j, k) \in \mathcal{V}\right\}$, where $\mathcal{V} \subseteq \mathbb{Z}_{+}^{2}$, and refer to Cohen, Daubechies and Vial (1993); Giné and Nickl (2016); Castillo (2014) for details. Each index $(j, k)$ is a pair where $j \geq 0$ is the wavelet level and $k$ the location index. The CDV basis is an orthogonal basis for $\mathbb{L}_{2}[0,1]$ equipped with the Lebesgue measure. We endow $\mathbb{L}_{2}[0,1]$ with the inner product $\langle f, g\rangle:=\int_{[0,1]} f g$. If $f \in B_{\infty, \infty}^{s}[0,1]$ for some $s>0$, then the wavelet series $\sum_{(j, k) \in \mathcal{V}}\left\langle f, \varphi_{j, k}\right\rangle \varphi_{j, k}$ converges uniformly to $f$. Conversely, for a given $\left(\theta_{j, k}\right)_{(j, k) \in \mathcal{V}}$, the series $\sum_{(j, k) \in \mathcal{V}} \theta_{j, k} \varphi_{j, k}$ converges uniformly if $\boldsymbol{\theta}$ is in

$$
\Theta \subseteq\left\{\boldsymbol{\theta} \in \mathbb{R}^{\mathcal{V}}: \sup _{x \in[0,1]} \sum_{(j, k) \in \mathcal{V}}\left|\theta_{j, k}\right|\left|\varphi_{j, k}(x)\right|<\infty\right\}
$$

Thus, we shall consider prior distributions over the space $\Theta$. Such prior distribution induces a prior distribution on the space of densities on $[0,1]$ (with respect to the Lebesgue measure) through the mapping $\boldsymbol{\theta} \mapsto p_{\boldsymbol{\theta}}$ such that

$$
p_{\boldsymbol{\theta}}(x):=\frac{\exp \left\{\sum_{(j, k) \in \mathcal{V}} \theta_{j, k} \varphi_{j, k}(x)\right\}}{\int_{[0,1]} \exp \left\{\sum_{(j, k) \in \mathcal{V}} \theta_{j, k} \varphi_{j, k}\right\}}, \quad x \in[0,1]
$$


The coefficients $\boldsymbol{\theta}$ in equation (2.1) are immediately seen to be the basis coefficients of an unnormalized version of the $\log$-density $\log p_{\boldsymbol{\theta}}$. By defining the $\log$-normalizing constant

$$
\xi^{\boldsymbol{\theta}}:=-\log \left(\int_{[0,1]} \exp \left\{\sum_{(j, k) \in \mathcal{V}} \theta_{j, k} \varphi_{j, k}\right\}\right) \in \mathbb{R}
$$

we can rewrite the $\log$-density $L_{\boldsymbol{\theta}}:=\log p_{\boldsymbol{\theta}}$ as

$$
L_{\boldsymbol{\theta}}=\xi^{\boldsymbol{\theta}}+\sum_{(j, k) \in \mathcal{V}} \theta_{j, k} \varphi_{j, k}=\sum_{(j, k) \in \mathcal{V}}\left(\theta_{j, k}+\left\langle\xi^{\boldsymbol{\theta}}, \varphi_{j, k}\right\rangle\right) \varphi_{j, k}
$$

\subsection{Spike-and-Slab log-density priors}

To obtain adaptive and optimal rates of contraction, we consider the so-called spike-and-slab prior distribution over $\Theta$ (Mitchell and Beauchamp, 1988). For some weights $\left(\omega_{1}, \omega_{2}, \ldots\right) \in[0,1]^{\mathbb{N}}$

$$
\theta_{j, k} \stackrel{\text { ind }}{\sim} \begin{cases}\left(1-\omega_{j}\right) \delta_{0}+\omega_{j} Q_{j}(\cdot) & \text { if } 0 \leq j \leq \frac{\log (n)}{\log (2)} \\ \delta_{0} & \text { if } j>\frac{\log (n)}{\log (2)}\end{cases}
$$

Here $\delta_{0}$ is the point mass at zero and $Q_{j}$ are probability distributions on $\mathbb{R}$. We assume $Q_{j}$ have densities $q_{j}$ such that for some $0<s_{0}<1 / 2$ and for some density $f$, we have $q_{j}(x):=$ $2^{j\left(s_{0}+1 / 2\right)} f\left(2^{j\left(s_{0}+1 / 2\right)} x\right)$ for every $j \geq 0$. We write $F$ the probability distribution with density $f$. We further assume that there are $a_{1}, b_{1}, b_{2}, \mu_{\star}, x_{0}>0$ such that

$$
\begin{gathered}
a_{1} e^{-j b_{1}} \leq \omega_{j} \leq \min \left\{1 / 2,2^{-j\left(1+\mu_{\star}\right)}\right\}, \\
\sup _{x>1 \vee x_{0}} e^{b_{2} x}(1-F[-\log (x), \log (x)]) \leq 1 .
\end{gathered}
$$

In order to ensure that the prior puts enough mass on neighborhoods of the true log-density $L$, we also assume that for all $G>0$ there is $g>0$ such that

$$
\inf _{x \in[-G, G]} f(x) \geq g
$$

We note that the assumptions of equations (2.2) and (2.4) are classical in the literature for rates of contraction in supremum loss. The equation (2.3) is however very strong, but guarantees that $a$ priori $L_{\boldsymbol{\theta}}$ has wavelet coefficients of reasonable magnitude, which guarantees that the posterior concentrates on nice neighborhoods of $L$, see in particular Section S4. As an example of distribution $F$ that satisfy the requirements of equations (2.3) and (2.4), one could take the distribution of the random variable $\log (Z)$ where $Z$ has an inverse-Gaussian distribution (or any distribution on $\mathbb{R}_{+}$with an exponential behaviour both near 0 and $\infty$ ).

\subsection{Adaptation and optimality under supremum loss}

This paper considers adaptation over bounded Hölder-Zygmund balls, which we define below. First, we give a precise definition for the Hölder-Zygmund spaces of smoothness. 
Definition 2.1 (Hölder-Zygmund spaces). For any $s>0$, the Hölder-Zygmund space $B_{\infty, \infty}^{s}[0,1]$ is the space of uniformly continuous functions $f:[0,1] \rightarrow \mathbb{R}$ such that $\|f\|_{\infty, \infty, s}<\infty$, where $\|f\|_{\infty, \infty, s}:=\sup _{(j, k) \in \mathcal{V}} 2^{j(s+1 / 2)}\left|\left\langle f, \varphi_{j, k}\right\rangle\right|$.

We are now in position to define the bounded Hölder-Zygmund ball of log-densities with radius $R>0$ and smoothness $s>0$

$$
\Sigma(R, s):=\left\{L \in B_{\infty, \infty}^{s}[0,1]:\|L\|_{\infty, \infty, s} \leq R, \int_{[0,1]} \exp (L)=1\right\} .
$$

We prove in the supplemental that spike-and-slab log-density priors satisfying equations (2.2) to (2.4) achieve adaptive and nearly optimal posterior contraction rates $\varepsilon_{n}^{*}(s)$ over $\Sigma(R, s)$ under Hellinger loss. In particular, the next theorem is proven in Section S3.

Theorem 2.1. Let $\Pi$ be the spike-and-slab log-density prior satisfying equations (2.2) to (2.4) and let $\mathrm{H}(P, Q)$ denote the Hellinger distance between probability distributions $P$ and $Q$. Then for all $0<s_{0} \leq s \leq S$ and for all $R>0$ there exists a constant $M>0$ such that

$$
\lim _{n \rightarrow \infty} \sup _{L \in \Sigma(R, s)} \mathbb{E}_{L} \Pi\left(\boldsymbol{\theta}: \mathbf{H}\left(P_{\boldsymbol{\theta}}, P_{L}\right)>M \varepsilon_{n}^{*}(s) \mid \mathbf{X}_{n}\right)=0 .
$$

The main theorem of the paper establishes that spike-and-slab log-density priors can achieve the optimal posterior contraction rates if we consider the $\mathbb{L}_{\infty}$ loss. The rate is optimal when $s>1 / 2$, with a slight deterioration in the region of small smoothness.

Theorem 2.2. $\quad$ Let $\Pi$ be the spike-and-slab log-density prior satisfying equations (2.2) to (2.4). Also let $\tilde{\varepsilon}_{n}^{*}(s):=\varepsilon_{n}^{*}(s)$ if $s>1 / 2$ or $\tilde{\varepsilon}_{n}^{*}(s):=\log (n) \varepsilon_{n}^{*}(s)$ if $0<s \leq 1 / 2$. Then for all $0<s_{0} \leq s \leq S$ and for all $R>0$ there exists a constant $M>0$ such that

$$
\lim _{n \rightarrow \infty} \sup _{L \in \Sigma(R, s)} \mathbb{E}_{L} \Pi\left(\boldsymbol{\theta}:\left\|L_{\boldsymbol{\theta}}-L\right\|_{\infty}>M \tilde{\varepsilon}_{n}^{*}(s) \mid \mathbf{X}_{n}\right)=0
$$

We emphasize that the Theorem 2.2 also entails posterior concentration of $\left\|p_{\boldsymbol{\theta}}-p_{L}\right\|_{\infty}$ at rate $\tilde{\varepsilon}_{n}^{*}(s)$, and thus because $L \in \Sigma(R, s)$ implies that $e^{-R} \leq p_{L} \leq e^{R}$. Hence, $\left\|p_{\boldsymbol{\theta}}-p_{L}\right\|_{\infty}$ and $\left\|L_{\boldsymbol{\theta}}-L\right\|_{\infty}$ are equivalent distances when the latter is small enough. This assumption that $L$ belongs to a Hölder ball of smoothness is stronger than the classical frequentist assumption that only $p_{L}$ does. In particular, we see that it entails that $p_{L}$ is bounded from below, which is of great help in the proofs. Of course, this begs the question of what can be said when $p_{L}$ is smooth but not bounded from below, which is outside the scope of this paper. We note that assuming smoothness on $L$ rather than $p_{L}$ is classical in the Bayesian community (see Castillo, 2014).

The rest of the paper is organized as follows. In the Section 3 we establish the main notations and give the main ideas behind the proof of the Theorem 2.2. In particular, we give guidelines for the proof and state a central contraction result in Theorem 3.1 which is at the core of the proof of Theorem 2.2. Then, in Section 4 we discuss the main implications of our results. Finally, proofs are given in Sections 5 to 7 , respectively for the Theorem 2.2, the Theorem 3.1, and for auxiliary results. Many secondary proofs are deferred to the Section S5 of the supplemental. 


\section{Heuristic and main ideas behind the proof of Theorem 2.2}

\subsection{Notations}

We let $\mathbb{N}:=\{1,2, \ldots\}$ denote the set of natural numbers, and we let $\mathbb{Z}_{+}:=\{0,1, \ldots\}$ denote the set of positive integers. The symbols $\lesssim$ and $\gtrsim$ are used to denote inequalities up to generic constants. If $a \lesssim b$ and $b \lesssim a$, we write $a \asymp b$. For two sequences $\left(a_{n}\right)_{n \in \mathbb{Z}_{+}}$and $\left(b_{n}\right)_{n \in \mathbb{Z}_{+}}$, the notation $a_{n}=o\left(b_{n}\right)$ means $\limsup _{n \rightarrow \infty}\left|a_{n} / b_{n}\right|=0$, and $a_{n}=O\left(b_{n}\right)$ means $\limsup _{n \rightarrow \infty}\left|a_{n} / b_{n}\right|=C$ for some $C \geq 0$. For $a, b \in \mathbb{R}$, we let $a \wedge b$ denote the minimum of $a$ and $b$, and $a \vee b$ stands for the maximum.

Densities are understood with respect to the Lebesgue measure. Lower-case notations $p, q, \ldots$ are used to denote densities, while upper-case $P, Q, \ldots$ denote the corresponding distributions. Given a $\log$-density $L$ on $[0,1]$, we write $p_{L}:=\exp \{L\}$ the corresponding density and $P_{L}$ the corresponding distribution. When $L=L_{\boldsymbol{\theta}}$ for some $\theta \in \Theta$, we abbreviate $P_{\boldsymbol{\theta}}$ for $P_{L_{\boldsymbol{\theta}}}$, etc.

We see $\mathbf{X}_{n}=\left(X_{1}, \ldots, X_{n}\right)$ as the beginning of an infinite sequence $\mathbf{X}_{\infty}=\left(X_{1}, X_{2}, \ldots\right)$ defined on a measurable space $(\Omega, \mathcal{A})$ and such that under $L$, the variables $X_{1}, X_{2}, \ldots$ are independent and identically distributed (iid) with distribution $P_{L}$. We write $\mathbb{P}_{L}$ the distribution of $\mathbf{X}_{\infty}$, and we write indistinctly $\mathbb{E}_{L}$ the expectation under $\mathbb{P}_{L}$ or under $P_{L}$. We write $\mathbb{P}_{n}=n^{-1} \sum_{i=1}^{n} \delta_{X_{i}}$ the empirical measure of $\mathbf{X}_{n}$.

We use the standard definitions for the $\mathbb{L}_{p}$ spaces of (equivalence classes) of functions with finite $\|\cdot\|_{p}$ norm, with $\|f\|_{p}^{p}:=\int|f|^{p}$ if $1 \leq p<\infty$, and $\|f\|_{\infty}:=\operatorname{ess}_{\sup }|f(x)|$. We will also make use of the Hellinger distance between two probability distributions $P$ and $Q$ having respective densities $p$ and $q$, defined as $\mathrm{H}(P, Q):=\frac{1}{\sqrt{2}}\|\sqrt{p}-\sqrt{q}\|_{2}$.

\subsection{Change of parameterization}

For some integer $J_{0}$ to be chosen sufficiently large, we define $B_{0}:=\left\{(j, k) \in \mathcal{V}: j \leq J_{0}\right\}$. The indices in $B_{0}$ corresponds to small scales wavelets and will require special cares. To ease the proof, it is convenient to relabel the wavelets with indices not in $B_{0}$. We let $\psi: \mathbb{N} \rightarrow \mathcal{V} \backslash B_{0}$ be the bijection corresponding to the lexicographical reordering of the index set $\mathcal{V} \backslash B_{0}$; i.e. writing $\psi(m)=(j, k)$ and $\psi\left(m^{\prime}\right)=\left(j^{\prime}, k^{\prime}\right)$

$$
m \leq m^{\prime} \Longleftrightarrow\left(j<j^{\prime}\right) \text { or }\left(j=j^{\prime} \text { and } k \leq k^{\prime}\right)
$$

For all $m \geq 1$ we write $J_{m}:=\psi(m)_{1}$ the scale-index of the wavelet $\varphi_{\psi(m)}$. By construction $J_{1}=$ $J_{0}+1$ and $J_{1} \leq J_{2} \leq \ldots$ For proofs, it is also convenient to define $B_{m}:=\{\psi(m)\}$ for all $m \geq 1$.

Given this re-indexing of the wavelets, we are now in position to define a change or parameterization which is convenient for proofs. We pick an arbitrary reference log-density $L \in \Sigma(R, s)$, and we establish the concentration under $L$ by taking care that the results are uniform over $\Sigma(R, s)$. Given $L$, we let $\theta_{j, k}^{L}:=\left\langle L, \varphi_{j, k}\right\rangle$, and we define

$$
F_{m}^{\boldsymbol{\theta}}:=\sum_{(j, k) \in B_{m}}\left(\theta_{j, k}-\theta_{j, k}^{L}\right)\left(\varphi_{j, k}-\mathbb{E}_{L}\left[\varphi_{j, k}\right]\right)
$$

Clearly $L_{\boldsymbol{\theta}}-L$ can be written uniquely in term of the $\left(F_{m}^{\boldsymbol{\theta}}\right)_{m \geq 0}$, so that we might as well consider $\left(F_{m}^{\boldsymbol{\theta}}\right)_{m \geq 0}$ as the parameter of the model. For each $m \geq 0$ we will write $\mathcal{F}_{m}:=\operatorname{span}\left\{\varphi_{j, k}-\mathbb{E}_{L}\left[\varphi_{j, k}\right]\right.$ : 
$\left.(j, k) \in B_{m}\right\}$, and $\mathcal{F}:=\mathcal{F}_{1} \times \mathcal{F}_{2} \times \ldots$ the infinite cartesian product of these spaces. We also let $\mathcal{F}_{0}:=\left\{\boldsymbol{F} \in \mathcal{F}: \mathbb{E}_{L}\left[\exp \left\{\sum_{m>0} F_{m}\right\}\right]<\infty\right\}$ denote the subset of proper parameters. Then, we now parameterize the model by $\boldsymbol{F} \in \mathcal{F}_{0}$. Using the constraint that $\mathbb{E}_{L}\left[\exp \left\{L_{\boldsymbol{F}}-L\right\}\right]=1$, we determine that the $\log$-likehood of the model $\boldsymbol{F} \in \mathcal{F}_{0}$ is given by

$$
L_{\boldsymbol{F}}-L=\sum_{m \geq 0} F_{m}-\log \mathbb{E}_{L}\left[\exp \left\{\sum_{m \geq 0} F_{m}\right\}\right]
$$

\subsection{Guidelines for the proof of Theorem 2.2 and intermediate contraction results}

Here we present the main ideas behind the proof of Theorem 2.2 and the main intermediate results that are used in the proof. First of all, it is convenient to assume that the posterior concentrates on nice neighborhoods of $L$. We will prove that the posterior concentrates on the set

$$
\mathscr{C}_{*}:=\left\{\boldsymbol{F} \in \mathcal{F}: \sup _{x \in[0,1]} \sum_{m \geq 0}\left|F_{m}(x)\right| \leq \delta\right\},
$$

where $0<\delta \leq 1$ is a constant to be chosen sufficiently small. Once it has been shown the posterior is concentrated on $\mathscr{C}_{*}$, the analysis of the log-likehood difference $L_{\boldsymbol{F}}-L$ is easier. Posterior contraction on $\mathscr{C}_{*}$ can be obtained by the classical machinery à la Ghosal and van der Vaart (Ghosal, Ghosh and van der Vaart, 2000; Ghosal and van der Vaart, 2007b,a) and is essentially a corollary of the Theorem 2.1. It can be done using similar arguments as those already found in Castillo (2014); Rivoirard and Rousseau (2012), as we do in Section S5.1 to prove the following lemma.

Lemma 3.1. Let $\Pi$ be the prior described in Section 2.2. Then for all $0<s_{0} \leq s \leq S$, all $R>0$, and all $\delta>0$,

$$
\lim _{n \rightarrow \infty} \sup _{L \in \Sigma(R, s)} \mathbb{E}_{L} \Pi\left(\mathscr{C}_{*}^{c} \mid \mathbf{X}_{n}\right)=0
$$

To obtain $\mathbb{L}_{\infty}$ rates, the goal is to relate the distance $\left\|L_{\boldsymbol{F}}-L\right\|_{\infty}$ to the parameter $\boldsymbol{F}$. In particular, we shall seek to relate $\left\|L_{\boldsymbol{F}}-L\right\|_{\infty}$ to $\left\{\left\|F_{m}\right\|_{2}: m \geq 0\right\}$, which is motivated by the fact that $\left\{\left\|F_{m}\right\|_{2}\right.$ : $m \geq 0\}$ essentially drives the behaviour of the posterior distributions. The following lemma serves this purpose.

Lemma 3.2. Let $\boldsymbol{F} \in \mathscr{C}_{*}$. Then, there exists a universal constant $C>0$ such that for all choice of $J_{0}$ we have

$$
\left\|L_{\boldsymbol{F}}-L\right\|_{\infty} \leq C \sum_{j \geq J_{0}} 2^{j / 2} \sup _{m: J_{m}=j}\left\|F_{m}\right\|_{2}
$$

In view of Lemmas 3.1 and 3.2, to prove the Theorem 2.2 it is enough to prove that the posterior concentrates on a set where the rhs in Lemma 3.2 is smaller than a multiple constant of $\tilde{\varepsilon}_{n}^{*}(s)$. Our stretagy is to build a partition of $\mathcal{F}$, where on each part we have a fine control of $\left\{\left\|F_{m}\right\|_{2}: m \geq 0\right\}$. We build the partition $\left(\mathscr{S}_{I}\right)_{I \subseteq \mathbb{Z}_{+}}$, such that for every $I \subseteq \mathbb{Z}_{+}$,

$$
\mathscr{S}_{I}:=\left\{\boldsymbol{F} \in \mathcal{F}: m \in I \Longrightarrow\left\|F_{m}\right\|_{2}>H_{I}(m), \quad m \notin I \Longrightarrow\left\|F_{m}\right\|_{2} \leq H_{I}(m)\right\},
$$


where we choose $H_{m}(I)$ as follows. We let $\Gamma, \gamma>0$ be constants to be determined, and we define the optimal truncation level $j_{n} \equiv j_{n}(s)$ as the only integer satisfying

$$
\begin{cases}\gamma 2^{-\left(j_{n}+1\right)(s+1 / 2)}<\Gamma \sqrt{\log (n) / n} \leq \gamma 2^{-j_{n}(s+1 / 2)} & \text { if } s>1 / 2, \\ \gamma 2^{-\left(j_{n}+1\right)(s+1 / 2)}<\Gamma 2^{-j_{n} / 2} \varepsilon_{n}^{*}(s) \leq \gamma 2^{-j_{n}(s+1 / 2)} & \text { if } 0<s \leq 1 / 2 .\end{cases}
$$

Then, for $\xi>1$ also to be chosen accordingly,

$$
H_{I}(m):=\left\{\begin{array}{ll}
\Gamma \xi^{-\mathbf{1}_{0 \in I} \mathbf{1}_{m \neq 0} \rho_{m}} & \text { if } J_{m} \leq j_{n}, \\
\gamma 2^{-J_{m}(s+1 / 2)} & \text { if } J_{m}>j_{n}
\end{array}, \quad \rho_{m}:= \begin{cases}\sqrt{\log (n) / n} & \text { if } s>1 / 2, \\
2^{-J_{m} / 2} \varepsilon_{n}^{*}(s) & \text { if } 0<s \leq 1 / 2\end{cases}\right.
$$

At this point, it might look obscure why the definition of $H_{I}(m)$ differs according to whether $s>1 / 2$ or not, and also according to whether $0 \in I$ or not. The subtle reason of this choice will be found when proving the Theorem 3.1. In fact, we will require to control some covariance terms involving $F_{m}$ and $F_{m^{\prime}}$ (see also Section 6.3). The control of these covariance terms can get tricky, and this particular choice of $H_{I}(m)$ permits to obtain the desired control.

Since $H_{I}(m)$ is function of $I$, it is not immediate that $\left(\mathscr{S}_{I}\right)_{I \subseteq \mathbb{N}}$ is a proper partition. We establish this fact in the next lemma, together with a useful property of this partition.

Lemma 3.3. The collection $\left(\mathscr{S}_{I}\right)_{I \subseteq \mathbb{Z}_{+}}$is a partition of $\mathcal{F}$ and

$$
\boldsymbol{F} \in\left(\bigcup_{I \neq \varnothing} \mathscr{S}_{I}\right)^{c} \Longrightarrow\left\|F_{m}\right\|_{2} \leq \begin{cases}\Gamma \rho_{m} & \text { if } J_{m} \leq j_{n} \\ \gamma 2^{-J_{m}(s+1 / 2)} & \text { if } J_{m}>j_{n}\end{cases}
$$

The previous lemma is one of the key result. In conjunction with Lemmas 3.1 and 3.2, it implies the following corollary which is the starting point of the proof of Theorem 2.2.

Corollary 3.1. For all choice of $J_{0}, \Gamma, \gamma, \xi$ and for all $(R, s)$ there exists $M>0$ such that the following bound is true.

$$
\mathbb{E}_{L} \Pi\left(\boldsymbol{\theta}:\left\|L_{\boldsymbol{\theta}}-L\right\|_{\infty}>M \tilde{\varepsilon}_{n}^{*}(s) \mid \mathbf{X}_{n}\right) \leq \sum_{\substack{I \subseteq \mathbb{Z}_{+} \\ I \neq \varnothing}} \mathbb{E}_{L} \Pi\left(\mathscr{C}_{*} \cap \mathscr{S}_{I} \mid \mathbf{X}_{n}\right)
$$

Our strategy is then to bound each of the terms $\mathbb{E}_{L} \Pi\left(\mathscr{C}_{*} \cap \mathscr{S}_{I} \mid \mathbf{X}_{n}\right)$ for $I \neq \varnothing$; which is done in the Theorem 3.1 below. Interestingly, the technique is reminiscent to the classical testing approach of (Ghosal, Ghosh and van der Vaart, 2000; Ghosal and van der Vaart, 2007a,b) with extra cares. Large parts of the proofs of Theorems 2.2 and 3.1 rely on the fine tuning of the constants $\delta, J_{0}, \Gamma, \gamma, \xi$, as well as the relation between those constants, and also on taking $n$ sufficiently large. Since the proofs are quite long, it can be challenging to keep track all along of the constraints those constants must satisfy. To facilitate the understanding of the theorems and their proof, we summarize in the next assumption how $\delta, J_{0}, \Gamma, \gamma, \xi$ and $n$ must be taken at the end of the day for the theorems to hold true.

Assumption 1. We assume that there are constants $K_{0}, K_{1}, K_{2}, K_{3}, K_{4}>0$ eventually large and eventually depending on $(R, s)$ but solely on $(R, s)$, such that

1. $J_{0} \geq K_{0} \max \{1, \log (1 / \delta), \log (\gamma)\}$;

2. $\log (n) \geq K_{1} \max \left\{2^{J_{0}}, \delta^{-2}, \log (\Gamma), \log (\gamma), \log (\xi)\right\}$; 
3. $\xi \geq K_{2} \delta^{-1} \max \{1, \gamma\} 2^{J_{0}}$;

4. $\gamma \geq K_{3} \max \left\{1, \delta^{-1}\right\}$;

5. $\Gamma \geq \max \left\{\gamma, K_{4} 2^{J_{0}}, K_{4} \xi\right\}$.

The constant $\delta$ will be taken as small as needed.

Finally, bounding $\mathbb{E}_{L} \Pi\left(\mathscr{C}_{*} \cap \mathscr{S}_{I} \mid \mathbf{X}_{n}\right)$ rely on splitting the parameter $\boldsymbol{F}$ into two parts $\boldsymbol{F}=$ $\left(\boldsymbol{F}_{I}, \overline{\boldsymbol{F}}_{I}\right)$ where $\boldsymbol{F}_{I}:=\left(F_{m}\right)_{m \in I}$ and $\overline{\boldsymbol{F}}_{I}:=\left(F_{m}\right)_{m \notin I}$. The following functions will also be used:

$$
S_{\boldsymbol{F}_{I}}:=\sum_{m \in I} F_{m}, \text { and, } S_{\overline{\boldsymbol{F}}_{I}}:=\sum_{m \notin I} F_{m} .
$$

Theorem 3.1. Suppose Assumption 1 is satisfied with constants $K_{0}, K_{1}, K_{2}>0$ sufficiently large and let $\Pi$ be a prior such that $\boldsymbol{F}_{I}$ and $\overline{\boldsymbol{F}}_{I}$ are independent for all $I \subseteq \mathbb{Z}_{+}$. Then, there are constants $c_{0}, c_{1}, c_{2}, \delta_{0}>0$ such that for all $0<\delta \leq \delta_{0}$, for all $\frac{512 \cdot c_{0} \delta}{1+512 \cdot c_{0} \delta}<\alpha \leq 1 / 2$, and for all $I \subseteq \mathbb{Z}_{+}$,

$$
\mathbb{E}_{L} \Pi\left(\mathscr{C}_{*} \cap \mathscr{S}_{I} \mid \mathbf{X}_{n}\right) \leq\left\{\frac{3 \exp \left\{-c_{2} n \mathcal{E}_{I}^{2}+c_{1} 2^{J_{0}}|I|\right\}^{\alpha}}{\left(1-e^{-c_{2} n \mathcal{E}_{I}^{2}}\right)^{2 \alpha}}\left\{\frac{\Pi\left(\mathcal{A}_{I}\right)}{\Pi\left(\tilde{\mathcal{A}}_{I}\right)}\right\}^{1-\alpha}\right\}^{\frac{1}{1+2 \delta}}
$$

where $\mathcal{E}_{I}:=\inf \left\{\mathbb{E}_{L}\left[S_{\boldsymbol{F}_{I}}^{2}\right]^{1 / 2}: \boldsymbol{F} \in \mathscr{S}_{I}\right\}, \mathcal{A}_{I}:=\left\{\boldsymbol{F}_{I}:\left\|F_{m}\right\|_{2}>H_{I}(m)\right\}$, and $\tilde{\mathcal{A}}_{I}:=\left\{\boldsymbol{F}_{I}\right.$ : $\left.\mathbb{E}_{L}\left[S_{\boldsymbol{F}_{I}}^{2}\right] \leq \delta^{2} \mathcal{E}_{I}^{2},\left\|S_{\boldsymbol{F}_{I}}\right\|_{\infty} \leq \delta\right\}$; provided that $\Pi\left(\tilde{\mathcal{A}}_{I}\right)>0$ and $\mathcal{E}_{I}>0$ for all $I \subseteq \mathbb{Z}_{+}$.

We note that the fact that $\mathcal{E}_{I}>0$ and $\Pi\left(\tilde{\mathcal{A}}_{I}\right)>0$ for the spike-and-slab prior are consequences of Lemma 5.1 and Proposition 7.2 that will be established later. Also, we point out that in the whole paper we make the abuse of notations of writing $\Pi$ to denote the prior on $\boldsymbol{\theta}, L_{\boldsymbol{\theta}}, L_{\boldsymbol{F}}, \boldsymbol{F}$, as well as for the restricted parameters $\boldsymbol{F}_{I}$ or $\overline{\boldsymbol{F}}_{I}$. The proof of the Theorem 2.2 consists on specializing the bound of Theorem 3.1 to the spike-and-slab prior and using it in conjunction with Corollary 3.1 to conclude.

\section{Discussion}

The master theorem of Bayesian nonparametrics The current state-of-the-art method in calculating posterior contraction rates is the master theorem developed by Ghosal, Ghosh and van der Vaart (2000); Ghosal and van der Vaart (2007b); Shen et al. (2001). This theorem relies on two main ingredients:

- The existence of tests for the hypothesis $H_{0}: L^{\prime}=L$ against the alternative $H_{1}:\left\|L^{\prime}-L\right\|_{\infty}>$ $M \varepsilon_{n}^{*}(s)$, with Type I and Type II errors decreasing as $\exp \left\{-K n \varepsilon_{n}^{*}(s)^{2}\right\}$;

- The prior puts enough mass on certain Kullback-Leibler neighborhoods of $L$.

In the context of $\mathbb{L}_{\infty}$ contraction, it is known that the master theorem yields suboptimal contraction rates (Giné and Nickl, 2011; Hoffmann, Rousseau and Schmidt-Hieber, 2015; Yoo, Rousseau and Rivoirard, 2017). The issue is discussed thoroughly in (Hoffmann, Rousseau and Schmidt-Hieber, 2015; Yoo, Rousseau and Rivoirard, 2017): no test has enough power to obtain the optimal rate of contraction in $\mathbb{L}_{\infty}$. In particular, the Type II error has to decay polynomially in $n$, unless we deteriorate the rate. It is known that not all the alternative $H_{1}$ has to be tested - only a suitable sieve - but this does not help either to get optimal rates, the root of the problem being deeper. 
The arguments in Hoffmann, Rousseau and Schmidt-Hieber (2015); Yoo, Rousseau and Rivoirard (2017) are strong, and it is natural to ask what is wrong in the current paper such that the tests we use in the proofs of Theorems 2.2 and 3.1 permit optimal contraction rates. This indeed relies on the nature of the alternative we test. We are not constructing tests for $H_{1}:\left\|L^{\prime}-L\right\|_{\infty}>M \varepsilon_{n}^{*}(s)$, but instead for each $I \subseteq \mathbb{N}$, we build a test for $H_{1}: L^{\prime} \in\left\{L_{\boldsymbol{F}}: \boldsymbol{F} \in \mathscr{S}_{I}\right\}$. Those tests (see the proof of Lemma 6.2) have Type I and Type II errors decreasing as $\exp \left\{-K n \mathcal{E}_{I}^{2}\right\}$, which is typically polynomial in $n$ when $|I|$ is small, and thus not in contradiction with the arguments of the aforementioned papers. We remark that $|I|$ small corresponds exactly to those log-densities $L^{\prime}$ that can be far from $L$ in $\mathbb{L}_{\infty}$ but close in $\mathbb{L}_{2}$, and thus hard to separate. When $|I|$ gets large, however, the powers of the tests increase, which is what saves us.

The main drawback of the method is getting a sharp enough bound on the denominator of the Bayes rule, which seems hard to do beyond the scope of independent wavelet coefficients, or at least having a nice structure. Anyhow, we believe the approach of the current paper shows that the master theorem of Bayesian nonparametrics can be still useful for $\mathbb{L}_{\infty}$ contraction, giving some hope toward a general $\mathbb{L}_{\infty}$ contraction result of the same flavour.

Suboptimality when $0<s \leq 1 / 2$ The rates of Theorem 2.2 are slightly suboptimal in the region $0<$ $s \leq 1 / 2$. The problem is indeed not inherent to the spike-and-slab prior, and as such not surprising as it is known density estimation on the interval behave very differently when $0<s \leq 1 / 2$ or $s>1 / 2$, see for instance Brown et al. (1998). Our troubles come from the impossibility of taking $\rho_{m}=\sqrt{\log (n) / n}$ when $s \leq 1 / 2$ and we have instead to take a much larger threshold $\rho_{m}=2^{-J_{m} / 2} \varepsilon_{n}^{*}(s)$. The reasons for this impossibility are to be found in controlling some covariance terms when decomposing the likelihood, see Section 6.3. In fact, this exhibits a major difference on the strength of the result we prove here: in the case $s>1 / 2$ the control of the posterior is much tighter. In particular, we prove that every wavelet coefficients of $L_{\boldsymbol{\theta}}$ at level $j \leq j_{n}$ is within $\sqrt{\log (n) / n}$ distance of the coefficients of $L$ if $s>1 / 2$, while we are only able to get a distance of $2^{-j / 2} \varepsilon_{n}^{*}(s)$ otherwise (which is much larger when $j$ is small).

To the best of our knowledge, no method based on asymptotic expansions of the log-likelihood succeeded before in getting posterior $\mathbb{L}_{\infty}$ rates when $s \leq 1 / 2$. Thereby, the strategy developed here shed new light on our understanding of $\mathbb{L}_{\infty}$ contraction. In view of the recent result of Castillo and Mismer (2019), however, methods based on conjugacy arguments are able to obtain adaptivity and optimality over all $0<s \leq 1$, with no extra $\log (n)$ factor. This shows that we don't really understand yet enough the behaviour of the log-likelihood when $0<s \leq 1 / 2$, which should be investigated in a near future by the author.

Estimation of the derivatives The spike-and-slab prior of Section 2.2 also achieves optimal contraction rates for estimating the derivatives of the density. We remark that if $L$ has derivatives $L^{(r)}, r \geq 1$ integer, then $s \geq 1>1 / 2$. Then, in this case, investigation of the proof of Theorem 2.2 shows that the posterior contracts on the set $\left\{L^{\prime}:\left|\left\langle L^{\prime}-L, \varphi_{j, k}\right\rangle\right| \lesssim \sqrt{\log (n) / n} \mathbf{1}_{j \leq j_{n}}+2^{-j(s+1 / 2)} \mathbf{1}_{j>j_{n}} \forall(j, k) \in \mathcal{V}\right\}$. Then, it is a classical result that this implies for all $1 \leq r \leq s \leq \bar{S}$ with $r$ integer,

$$
\sup _{L \in \Sigma(R, s)} \mathbb{E}_{L} \Pi\left(\boldsymbol{\theta}:\left\|L_{\boldsymbol{\theta}}^{(r)}-L^{(r)}\right\|_{\infty}>M \varepsilon_{n}^{*}(s)^{\frac{s-r}{s}} \mid \mathbf{X}_{n}\right)=o(1) .
$$




\section{Proof of the Theorem 2.2}

As explained in Section 3.3, the proof of Theorem 2.2 consists on plugging the bound of Theorem 3.1 into the bound of Corollary 3.1. The first step is to obtain an upper estimate on $\Pi\left(\mathcal{A}_{I}\right) / \Pi\left(\tilde{\mathcal{A}}_{I}\right)$. The next lemma is proved in Section S5.3.

Lemma 5.1. Suppose Assumption 1 is satisfied with constants $K_{0}, K_{1}, K_{2}, K_{3}>0$ sufficiently large. Then, there are universal constants $\nu_{1}, \nu_{2}>0$ such that for all $I \subseteq \mathbb{Z}_{+}$,

$$
\frac{\Pi\left(\mathcal{A}_{I}\right)}{\Pi\left(\tilde{\mathcal{A}}_{I}\right)} \leq \nu_{1} \exp \left\{\nu_{2} \log (n) \sum_{m \in I} 2^{J_{0} \mathbf{1}_{m=0}} \mathbf{1}_{J_{m} \leq j_{n}}-\left(1+\mu_{\star}\right) \log (2) \sum_{m \in I} J_{m} \mathbf{1}_{J_{m}>j_{n}}\right\} .
$$

Furthermore, if $I \cap\left\{m: J_{m}>\log (n) / \log (2)\right\} \neq \varnothing$, then $\Pi\left(\mathcal{A}_{I}\right) / \Pi\left(\tilde{\mathcal{A}}_{I}\right)=0$.

Then, we can plug the bound of Lemma 5.1 into the Theorem 3.1 and fine-tune $\alpha$ in function of $I$ to obtain a clean bound on $\mathbb{E}_{L} \Pi\left(\mathscr{C}_{*} \cap \mathscr{S}_{I} \mid \mathbf{X}_{n}\right)$. We do so in the following lemma, also proved in Section S5.3.

Lemma 5.2. Suppose Assumption 1 is satisfied with constants $K_{0}, K_{1}, K_{2}, K_{3}, K_{4}>0$ sufficiently large and $\delta>0$ is taken sufficiently small. Then, there are universal constants $\nu_{3}, \nu_{4}>0$ such that for all $I \subseteq \mathbb{Z}_{+}$,

$$
\mathbb{E}_{L} \Pi\left(\mathscr{C}_{*} \cap \mathscr{S}_{I} \mid \mathbf{X}_{n}\right) \leq \nu_{4} \prod_{\substack{m \in I \\ J_{m} \leq j_{n}}} n^{-\nu_{3} K_{4}^{2}} \prod_{\substack{m \in I \\ J_{m}>j_{n}}} 2^{-J_{m}\left(1+\mu_{\star} / 2\right)}
$$

Now we are in position to use the bound established in Lemma 5.2 with the inequality of Corollary 3.1 to finish the proof. Define for simplicity $g_{m}:=\nu_{3} K_{4}^{2} \log (n)$ if $0 \leq J_{m} \leq j_{n}$ and $g_{m}:=$ $J_{m}\left(1+\mu_{\star} / 2\right) \log (2)$ if $J_{m}>j_{n}$. Then,

$$
\begin{aligned}
\sum_{\substack{I \subseteq \mathbb{Z}_{+} \\
I \neq \varnothing}} \mathbb{E}_{L} \Pi\left(\mathscr{C}_{*} \cap \mathscr{S}_{I} \mid \mathbf{X}_{n}\right) & \leq \nu_{4} \sum_{\mathbf{b} \in\{0,1\}^{\mathbb{Z}_{+}}} \mathbf{1}\left\{\sum_{m} b_{m} \geq 1\right\} \prod_{m \in \mathbb{Z}_{+}} e^{-g_{m} b_{m}} \\
& \leq \nu_{4} \sum_{m^{\prime} \in \mathbb{Z}_{+}} \sum_{\mathbf{b} \in\{0,1\}^{\mathbb{Z}_{+}}} b_{m^{\prime}} \prod_{m \in \mathbb{Z}_{+}} e^{-g_{m} b_{m}} \\
& =\nu_{4} \sum_{m^{\prime} \in \mathbb{Z}_{+}} \sum_{\substack{\mathbf{b} \in\{0,1\}^{\mathbb{Z}_{+}} \\
\sum_{m^{\prime}}}} e^{-g_{m^{\prime}} b_{m^{\prime}} b_{m^{\prime}}} \prod_{\substack{m \in \mathbb{Z}_{+} \\
m \neq m^{\prime}}} e^{-g_{m} b_{m}} \\
& =\nu_{4} \sum_{\substack { m^{\prime} \in \mathbb{Z}_{+} \\
\begin{subarray}{c}{m \in \mathbb{Z}_{+} \\
m \neq m^{\prime}{ m ^ { \prime } \in \mathbb { Z } _ { + } \\
\begin{subarray} { c } { m \in \mathbb { Z } _ { + } \\
m \neq m ^ { \prime } } }\end{subarray}}\left(1+e^{-g_{m}}\right) .
\end{aligned}
$$

Hence we get the bound,

$$
\sum_{\substack{I \subseteq \mathbb{Z}_{+} \\ I \neq \varnothing}} \mathbb{E}_{L} \Pi\left(\mathscr{C}_{*} \cap \mathscr{S}_{I} \mid \mathbf{X}_{n}\right) \leq \nu_{4} \exp \left(\sum_{m \in \mathbb{Z}_{+}} e^{-g_{m}}\right) \sum_{m \in \mathbb{Z}_{+}} e^{-g_{m}}
$$


The previous display is $o(1)$ whenever $\sum_{m \in \mathbb{Z}_{+}} e^{-g_{m}}=o(1)$, which we prove now. Indeed,

$$
\begin{aligned}
\sum_{m \in \mathbb{Z}_{+}} e^{-g_{m}} & =\sum_{m \in \mathbb{Z}_{+}} e^{-g_{m}} \mathbf{1}_{J_{m} \leq j_{n}}+\sum_{m \in \mathbb{Z}_{+}} e^{-g_{m}} \mathbf{1}_{J_{m}>j_{n}} \\
& \lesssim n^{-\nu_{3} K_{4}^{2}} \sum_{m \in \mathbb{Z}_{+}} \mathbf{1}_{J_{m} \leq j_{n}}+\sum_{m \in \mathbb{Z}_{+}} 2^{-j\left(1+\mu_{\star} / 2\right)} \mathbf{1}_{J_{m}>j_{n}} \\
& \lesssim n^{-\nu_{3} K_{4}^{2}} \sum_{j=0}^{j_{n}} 2^{j}+\sum_{j>j_{n}} 2^{-j\left(1+\mu_{\star} / 2\right)} 2^{j} \\
& \lesssim n^{-\nu_{3} K_{4}^{2}} 2^{j_{n}}+2^{-j_{n} \mu_{\star} / 2}
\end{aligned}
$$

where the third line follows as there are no more than $\lesssim 2^{j}$ wavelets at level $j$. Now, we remark that $2^{j_{n}} \asymp(n / \log (n))^{2 /(2 s+1)}$. Hence, if $K_{4}$ is taken large enough, $\sum_{m \in \mathbb{Z}_{+}} e^{-g_{m}}=o(1)$, as claimed.

\section{Proof of the Theorem 3.1}

\subsection{Main ideas}

We already know that $\mathscr{S}_{I} \subseteq\left\{\boldsymbol{F}_{I}: \mathbb{E}_{L}\left[S_{\boldsymbol{F}_{I}}^{2}\right] \geq \mathcal{E}_{I}^{2}\right\}$ by construction. We obtain a finer result by further slicing the set $\mathscr{S}_{I}$. For $y \geq 1$ integer, we let

$$
\mathscr{S}_{I}^{y}:=\mathscr{S}_{I} \cap\left\{\boldsymbol{F}: y \mathcal{E}_{I}^{2} \leq \mathbb{E}_{L}\left[S_{\boldsymbol{F}_{I}}^{2}\right]<(y+1) \mathcal{E}_{I}^{2}\right\} .
$$

Similarly, we define we define $\mathcal{A}_{I}^{y}:=\mathcal{A}_{I} \cap\left\{\boldsymbol{F}_{I}: y \mathcal{E}_{I}^{2} \leq \mathbb{E}_{L}\left[S_{\boldsymbol{F}_{I}}^{2}\right]<(1+y) \mathcal{E}_{I}^{2}\right\}$. Clearly $\left(\mathcal{A}_{I}^{y}\right)_{y \geq 1}$ is a partition of $\mathcal{A}_{I}$. The first lemma establishes a first bound on the posterior mass of $\mathscr{S}_{I}^{y}$.

Lemma 6.1. Suppose Assumption 1 is satisfied with constants $K_{0}, K_{1}, K_{2}>0$ sufficiently large. Also suppose $\Pi$ is such that $\boldsymbol{F}_{I}$ and $\overline{\boldsymbol{F}}_{I}$ are independent for all $I \subseteq \mathbb{N}$, and $\Pi\left(\tilde{\mathcal{A}}_{I}\right)>0$ for all $I \subseteq \mathbb{Z}_{+}$. Then, there are universal constants $c_{0}, \delta_{0}>0$ such that for all $t>0$ there is an event $\Omega_{t}$ with $\mathbb{P}_{L}^{n}\left(\Omega_{t}^{c}\right) \leq e^{-t}$ and if $\mathbf{X}_{n} \in \Omega_{t}$, for all $0<\delta \leq \delta_{0}$, for all $I \subseteq \mathbb{Z}_{+}$, for all $y \geq 1$,

$$
\Pi\left(\mathscr{C}_{*} \cap \mathscr{S}_{I}^{y} \mid \mathbf{X}_{n}\right) \leq 2 e^{2 \delta t+c_{0} \delta y n \mathcal{E}_{I}^{2}} \int_{\mathcal{A}_{I}^{y} \cap\left\{\left\|S_{\boldsymbol{F}_{I}}\right\|_{\infty} \leq \eta\right\}} \prod_{i=1}^{n} \frac{q_{\boldsymbol{F}_{I}}\left(X_{i}\right)}{p_{L}\left(X_{i}\right)} \frac{\Pi\left(\mathrm{d} \boldsymbol{F}_{I}\right)}{\Pi\left(\tilde{\mathcal{A}}_{I}\right)},
$$

where $q_{\boldsymbol{F}_{I}}$ is a probability density on $[0,1]$ whose exact expression is known but deferred to the proof of the lemma for convenience.

The last lemma is the key result of the proof. Interestingly, the classical approach to concentration rates à la Ghosal, Ghosh and van der Vaart (2000) consists on establishing a similar relation, but with $q_{\boldsymbol{F}_{I}}$ replaced by $\exp \left\{L_{\boldsymbol{F}}\right\}$ and $\tilde{\mathcal{A}}_{I}$ replaced by a Kullback-Leibler neighborhood of $p_{L}$. We use the estimate of Lemma 6.1 to bound $\mathbb{E}_{L} \Pi\left(\mathscr{C}_{*} \cap \mathscr{S}_{I}^{y} \mid \mathbf{X}_{n}\right)$ using the standard testing approach à la Ghosal, Ghosh and van der Vaart (2000), coupled with the square-root trick of Lijoi, Prünster and Walker (2005); Walker, Lijoi and Prünster (2007); Ghosal and van der Vaart (2007a). This step is rather immediate in view of the existing literature and it boils down to bound $\inf \left\{\mathrm{H}\left(Q_{S_{F_{I}}}, P_{L}\right)^{2}\right.$ : $\left.\boldsymbol{F}_{I} \in \mathcal{A}_{I}^{y},\left\|S_{\boldsymbol{F}_{I}}\right\|_{\infty} \leq \delta\right\}$ and the metric entropy (in the Hellinger distance) of the set of densities $\mathcal{P}_{I}^{y}:=\left\{q_{\boldsymbol{F}_{I}}: \boldsymbol{F}_{I} \in \mathcal{A}_{I}^{y},\left\|S_{\boldsymbol{F}_{I}}\right\|_{\infty} \leq \delta\right\}$. This gives the following lemma. 
Lemma 6.2. Suppose Assumption 1 is satisfied with constants $K_{0}, K_{1}, K_{2}>0$ sufficiently large, and let everything as in Lemma 6.1. Then, there are universal constants $c_{1}, \delta_{0}>0$ such that for all $0<\delta \leq \delta_{0}$, for all $I \subseteq \mathbb{Z}_{+}$, for all $y \geq 1$, for all $t>0$,

$$
\mathbb{E}_{L}\left[\Pi\left(\mathscr{C}_{*} \cap \mathscr{S}_{I}^{y} \mid \mathbf{X}_{n}\right) \mathbf{1}_{\Omega_{t}}\right] \leq\left\{\frac{8 \exp \left(-\frac{y n \mathcal{E}_{I}^{2}}{256}+2 \delta t+c_{1} 2^{J_{0}}|I|\right)}{1-e^{-y n \mathcal{E}_{I}^{2} / 256}} \frac{\Pi\left(\mathcal{A}_{I}^{y}\right)}{\Pi\left(\tilde{\mathcal{A}}_{I}\right)}\right\}^{1 / 2} .
$$

We can obtain a bound on $\mathbb{E}_{L}\left[\Pi\left(\mathscr{C}_{*} \cap \mathscr{S}_{I} \mid \mathbf{X}_{n}\right) \mathbf{1}_{\Omega_{t}}\right]$ by summing over $y \geq 1$ the bound obtained in Lemma 6.2. This gives a valid bound, but it is in not sharp enough in cases where $|I|$ gets too large or $n \mathcal{E}_{I}^{2}$ is too small. Indeed, in those cases, we can improve the bound to give more importance to the prior by remarking that taking the expectation both sides of the expression in Lemma 6.1 and applying Fubini's theorem gives

$$
\mathbb{E}_{L}\left[\Pi\left(\mathscr{C}_{*} \cap \mathscr{S}_{I}^{y} \mid \mathbf{X}_{n}\right) \mathbf{1}_{\Omega_{t}}\right] \leq 2 e^{2 \delta t+c_{0} \delta y n \mathcal{E}_{I}^{2}} \frac{\Pi\left(\mathcal{A}_{I}^{y}\right)}{\Pi\left(\tilde{\mathcal{A}}_{I}\right)} .
$$

This improvement permits to assume only $\mu_{\star}>0$, otherwise we would have to assume $\mu_{\star}>1$, which may be undesirable in practice (as it may cause over-shrinkage). The next lemma leverages that $\mathbb{E}_{L}\left[\Pi\left(\mathscr{C}_{*} \cap \mathscr{S}_{I}^{y} \mid \mathbf{X}_{n}\right) \mathbf{1}_{\Omega_{t}}\right]$ is bounded above by the minimum between the expression in Lemma 6.2 and the last display to get a sharp bound on $\mathbb{E}_{L}\left[\Pi\left(\mathscr{C}_{*} \cap \mathscr{S}_{I} \mid \mathbf{X}_{n}\right) \mathbf{1}_{\Omega_{t}}\right]$.

Lemma 6.3. Suppose Assumption 1 is satisfied with constants $K_{0}, K_{1}, K_{2}>0$ sufficiently large, and let everything as in Lemmas 6.1 and 6.2. Then, there are universal constants $c_{2}, \delta_{0}>0$ such that for all $0<\delta \leq \delta_{0}$, for all $I \subseteq \mathbb{Z}_{+}$, for all $\frac{512 \cdot c_{0} \delta}{1+512 \cdot c_{0} \delta}<\alpha \leq 1 / 2$, for all $t>0$,

$$
\mathbb{E}_{L}\left[\Pi\left(\mathscr{C}_{*} \cap \mathscr{S}_{I} \mid \mathbf{X}_{n}\right) \mathbf{1}_{\Omega_{t}}\right] \leq \frac{\sqrt{8} e^{2 \delta t} \exp \left\{-c_{2} n \mathcal{E}_{I}^{2}+c_{1} 2^{J_{0}}|I|\right\}^{\alpha}}{\left(1-e^{-c_{2} n \mathcal{E}_{I}^{2}}\right)^{2 \alpha}}\left\{\frac{\Pi\left(\mathcal{A}_{I}\right)}{\Pi\left(\tilde{\mathcal{A}}_{I}\right)}\right\}^{1-\alpha}
$$

Finally, to obtain the bound in the statement of the theorem, we note that,

$$
\mathbb{E}_{L} \Pi\left(\mathscr{C}_{*} \cap \mathscr{S}_{I} \mid \mathbf{X}_{n}\right) \leq \inf _{t>0}\left\{e^{-t}+\mathbb{E}_{L}\left[\Pi\left(\mathscr{C}_{*} \cap \mathscr{S}_{I} \mid \mathbf{X}_{n}\right) \mathbf{1}_{\Omega_{t}}\right]\right\}
$$

Plugging the bound obtained in Lemma 6.3 into the previous display and solving to find the infimum gives the bound of the theorem when choosing $\delta$ small enough.

\subsection{Proofs of Lemmas 6.1 to 6.3}

Proof of Lemma 6.1. Let define $\Phi(f):=f-\mathbb{E}_{L}[f]-\log \mathbb{E}_{L}\left[e^{f-\mathbb{E}_{L}[f]}\right]$ and $C(f, g):=\log \mathbb{E}_{L}\left[e^{\Phi(f)} e^{\Phi(g)}\right]$. We will see that $-\mathrm{C}\left(S_{\boldsymbol{F}_{I}}, S_{\overline{\boldsymbol{F}}_{I}}\right)$ is asymptotically equivalent to the covariance of $S_{\boldsymbol{F}_{I}}$ and $S_{\overline{\boldsymbol{F}}_{I}}$, and thus we will refer abusively to this term as the covariance from now on. It is easily seen that the $\log$-likelihood can be rewritten as

$$
L_{\boldsymbol{F}}-L=\Phi\left(S_{\boldsymbol{F}_{I}}\right)+\Phi\left(S_{\overline{\boldsymbol{F}}_{I}}\right)-\mathrm{C}\left(S_{\boldsymbol{F}_{I}}, S_{\overline{\boldsymbol{F}}_{I}}\right) .
$$

Then, by the Bayes rule,

$$
\Pi\left(\mathscr{C}_{*} \cap \mathscr{S}_{I}^{y} \mid \mathbf{X}_{n}\right)=\frac{\int_{\mathscr{C}_{*} \cap \mathscr{F}_{I}^{y}} e^{n \mathbb{P}_{n} \Phi\left(S_{\boldsymbol{F}_{I}}\right)} e^{n \mathbb{P}_{n} \Phi\left(S_{\overline{\boldsymbol{F}}_{I}}\right)} e^{-n \mathrm{C}\left(S_{\boldsymbol{F}_{I}}, S_{\overline{\boldsymbol{F}}_{I}}\right)} \Pi(\mathrm{d} \boldsymbol{F})}{\int e^{n \mathbb{P}_{n} \Phi\left(S_{\boldsymbol{F}_{I}}\right)} e^{n \mathbb{P}_{n} \Phi\left(S_{\overline{\boldsymbol{F}}_{I}}\right)} e^{-n \mathrm{C}\left(S_{\boldsymbol{F}_{I}}, S_{\overline{\boldsymbol{F}}_{I}}\right)} \Pi(\mathrm{d} \boldsymbol{F})} .
$$


Recall that $\boldsymbol{F}_{I}:=\left(F_{m}\right)_{m \in I}$ and $\overline{\boldsymbol{F}}_{I}:=\left(F_{m}\right)_{m \notin I}$. Also, in addition to $\mathcal{A}_{I}^{y}$, we let

$$
\mathcal{N}_{I}:=\left\{\overline{\boldsymbol{F}}_{I}: \forall m \notin I,\left\|F_{m}\right\|_{2} \leq H_{I}(m)\right\} .
$$

It is immediate that if $\boldsymbol{F} \in \mathscr{S}_{I}^{y}$ then $\boldsymbol{F}_{I} \in \mathcal{A}_{I}^{y}$ and $\overline{\boldsymbol{F}}_{I} \in \mathcal{N}_{I}$. Also, if $\boldsymbol{F} \in \mathscr{C}_{*}$, then

$$
\max \left\{\left|S_{\boldsymbol{F}_{I}}(x)\right|,\left|S_{\overline{\boldsymbol{F}}_{I}}(x)\right|\right\} \leq \max \left\{\sum_{m \in I}\left|F_{m}(x)\right|, \sum_{m \notin I}\left|F_{m}(x)\right|\right\} \leq \sum_{m \geq 0}\left|F_{m}(x)\right| \leq \delta .
$$

So for all $\boldsymbol{F} \in \mathscr{C}_{*}$ and for all $I \subseteq \mathbb{Z}_{+}$, we have $\left\|S_{\boldsymbol{F}_{I}}\right\|_{\infty} \leq \delta$ and $\left\|S_{\overline{\boldsymbol{F}}_{I}}\right\|_{\infty} \leq \delta$. It follows by equation (6.3) that $\Pi\left(\mathscr{C}_{*} \cap \mathscr{S}_{I}^{y} \mid \mathbf{X}_{n}\right)$ is bounded from above by

$$
\frac{\int \mathbf{1}_{\mathcal{A}_{I}^{y}}\left(\boldsymbol{F}_{I}\right) \mathbf{1}_{\mathcal{N}_{I}}\left(\overline{\boldsymbol{F}}_{I}\right) \mathbf{1}_{\left\|S_{\boldsymbol{F}_{I}}\right\|_{\infty} \leq \delta} \mathbf{1}_{\| S_{\overline{\boldsymbol{F}}_{I}}} \|_{\infty} \leq \delta e^{n \mathbb{P}_{n} \Phi\left(S_{\boldsymbol{F}_{I}}\right)} e^{n \mathbb{P}_{n} \Phi\left(S_{\overline{\boldsymbol{F}}_{I}}\right)} e^{-n \mathrm{C}\left(S_{\boldsymbol{F}_{I}}, S_{\overline{\boldsymbol{F}}_{I}}\right)} \Pi(\mathrm{d} \boldsymbol{F})}{\int \mathbf{1}_{\tilde{\mathcal{A}}_{I}}\left(\boldsymbol{F}_{I}\right) \mathbf{1}_{\mathcal{N}_{I}}\left(\overline{\boldsymbol{F}}_{I}\right) \mathbf{1}_{\left\|S_{\boldsymbol{F}_{I}}\right\|_{\infty} \leq \delta} \mathbf{1}_{\left\|S_{\overline{\boldsymbol{F}}_{I}}\right\|_{\infty} \leq \delta} e^{n \mathbb{P}_{n} \Phi\left(S_{\boldsymbol{F}_{I}}\right)} e^{n \mathbb{P}_{n} \Phi\left(S_{\overline{\boldsymbol{F}}_{I}}\right)} e^{-n \mathrm{C}\left(S_{\boldsymbol{F}_{I}}, S_{\overline{\boldsymbol{F}}_{I}}\right)} \Pi(\mathrm{d} \boldsymbol{F})} .
$$

The main challenge in the proof of the theorem is to control the term $\mathrm{C}\left(S_{\boldsymbol{F}_{I}}, S_{\overline{\boldsymbol{F}}_{I}}\right)$ both in the numerator and denominator, which is deferred to Section 6.3. In fact, by Corollaries 6.1 and 6.2, if the constants $K_{0}, K_{1}, K_{2}$ in Assumption 1 are taken sufficiently large, there is a universal $C>0$ such that taking $\delta$ small enough gives

$$
2 e^{C \delta y n \mathcal{E}_{I}^{2}} \frac{\int \mathbf{1}_{\mathcal{A}_{I}^{y}}\left(\boldsymbol{F}_{I}\right) \mathbf{1}_{\mathcal{N}_{I}}\left(\overline{\boldsymbol{F}}_{I}\right) \mathbf{1}_{\left\|S_{\boldsymbol{F}_{I}}\right\|_{\infty} \leq \delta} \mathbf{1}_{\left\|S_{\overline{\boldsymbol{F}}_{I}}\right\|_{\infty} \leq \delta} e^{n \mathbb{P}_{n} \Phi\left(S_{\boldsymbol{F}_{I}}\right)} e^{n \mathbb{P}_{n} \Phi\left(S_{\overline{\boldsymbol{F}}_{I}}\right)} \Pi(\mathrm{d} \boldsymbol{F})}{\int \mathbf{1}_{\tilde{\mathcal{A}}_{I}}\left(\boldsymbol{F}_{I}\right) \mathbf{1}_{\mathcal{N}_{I}}\left(\overline{\boldsymbol{F}}_{I}\right) \mathbf{1}_{\left\|S_{\boldsymbol{F}_{I}}\right\|_{\infty} \leq \delta} \mathbf{1}_{\left\|S_{\overline{\boldsymbol{F}}_{I}}\right\|_{\infty} \leq \delta} e^{n \mathbb{P}_{n} \Phi\left(S_{\boldsymbol{F}_{I}}\right)} e^{n \mathbb{P}_{n} \Phi\left(S_{\overline{\boldsymbol{F}}_{I}}\right)} \Pi(\mathrm{d} \boldsymbol{F})},
$$

But $\boldsymbol{F}_{I}$ is independent of $\overline{\boldsymbol{F}}_{I}$ and $S_{\boldsymbol{F}_{I}}$ is solely function of $\boldsymbol{F}_{I}$ (respectively $S_{\overline{\boldsymbol{F}}_{I}}$ and $\overline{\boldsymbol{F}}_{I}$ ), thus

$$
\begin{aligned}
\Pi\left(\mathscr{C}_{*} \cap \mathscr{S}_{I}^{y} \mid \mathbf{X}_{n}\right) & \leq 2 e^{C \delta y n \mathcal{E}_{I}^{2}} \frac{\int_{\mathcal{A}_{I}^{y}} \mathbf{1}_{\left\|S_{\boldsymbol{F}_{I}}\right\|_{\infty} \leq \delta} e^{n \mathbb{P}_{n} \Phi\left(S_{\boldsymbol{F}_{I}}\right)} \Pi\left(\mathrm{d} \boldsymbol{F}_{I}\right)}{\int_{\tilde{\mathcal{A}}_{I}} \mathbf{1}_{\left\|S_{\boldsymbol{F}_{I}}\right\|_{\infty} \leq \delta} e^{n \mathbb{P}_{n} \Phi\left(S_{\boldsymbol{F}_{I}}\right)} \Pi\left(\mathrm{d} \boldsymbol{F}_{I}\right)} \\
& =2 e^{C \delta y n \mathcal{E}_{I}^{2}} \frac{\int_{\mathcal{A}_{I}^{y}} \mathbf{1}_{\left\|S_{\boldsymbol{F}_{I}}\right\|_{\infty} \leq \delta} e^{n \mathbb{P}_{n} \Phi\left(S_{\boldsymbol{F}_{I}}\right)} \Pi\left(\mathrm{d} \boldsymbol{F}_{I}\right)}{\int_{\tilde{\mathcal{A}}_{I}} e^{n \mathbb{P}_{n} \Phi\left(S_{\boldsymbol{F}_{I}}\right)} \Pi\left(\mathrm{d} \boldsymbol{F}_{I}\right)}
\end{aligned}
$$

where the second line follows because $\boldsymbol{F}_{I} \in \tilde{\mathcal{A}}_{I} \Longrightarrow\left\|S_{\boldsymbol{F}_{I}}\right\|_{\infty} \leq \delta$ by construction. It is interesting that $x \mapsto p_{L}(x) e^{\Phi S_{F_{I}}(x)}$ is indeed a proper density function, i.e it is non-negative and integrates to 1 . We write $q_{\boldsymbol{F}_{I}}(x):=p_{L}(x) e^{\Phi S_{\boldsymbol{F}_{I}}(x)}$. We then can bound the expectation of equation (6.4) using the standard approach à la Ghosal, Ghosh and van der Vaart. In particular, we arrive at the bound of equation (6.1) by controlling the $\mathbb{P}_{L}$-probability of the event

$$
\Omega_{t}:=\left\{\mathbf{X}_{n}: \int_{\tilde{\mathcal{A}}_{I}} \prod_{i=1}^{n} \frac{q_{F_{I}}\left(X_{i}\right)}{p_{L}\left(X_{i}\right)} \frac{\Pi\left(\mathrm{d} \boldsymbol{F}_{I}\right)}{\Pi\left(\tilde{\mathcal{A}}_{I}\right)} \geq e^{-n \delta^{2} \mathcal{E}_{I}^{2}} e^{-\sqrt{2 n \delta^{2} \mathcal{E}_{I}^{2} t}-\delta t}\right\} .
$$

Proposition 6.1. Let $\Pi$ be any probability measure supported on the set $\tilde{\mathcal{A}}_{I}$. For all $I \subseteq \mathbb{Z}_{+}$, for all $0<\delta \leq \log (2)$, for all $t>0$, and for all $n>0, \mathbb{P}_{L}^{n}\left(\Omega_{t}\right) \geq 1-e^{-t}$. 
Then, on the event that $\mathbf{X}_{n} \in \Omega_{t}$, the equation (6.4) becomes

$$
\Pi\left(\mathscr{C}_{*} \cap \mathscr{S}_{I}^{y} \mid \mathbf{X}_{n}\right) \leq 2 e^{C \delta n y \mathcal{E}_{I}^{2}+n \delta^{2} \mathcal{E}_{I}^{2}+\sqrt{2 n \delta^{2} \mathcal{E}_{I}^{2} t}+\delta t} \int_{\mathcal{A}_{I}^{y} \cap\left\{\left\|S_{\boldsymbol{F}_{I}}\right\|_{\infty} \leq \eta\right\}} \prod_{i=1}^{n} \frac{q_{\boldsymbol{F}_{I}}\left(X_{i}\right)}{p_{L}\left(X_{i}\right)} \frac{\Pi\left(\mathrm{d} \boldsymbol{F}_{I}\right)}{\Pi\left(\tilde{\mathcal{A}}_{I}\right)} .
$$

The conclusion follows because $y \geq 1$, and because if $t \leq 2 n \mathcal{E}_{I}^{2}$ then $\sqrt{2 n \delta^{2} \mathcal{E}_{I}^{2} t}+\delta t \leq 2 \delta n \mathcal{E}_{I}^{2}+\delta t$, while if $t>2 n \mathcal{E}_{I}^{2}$ then $\sqrt{2 n \delta^{2} \mathcal{E}_{I}^{2} t}+\delta t \leq 2 \delta t$. Hence we can take $\delta_{0}=\log (2)$ and $c_{0}=C+2+\delta_{0}$.

Proof of Lemma 6.2. First we obtain a lower bound on $\inf \left\{\mathrm{H}\left(Q_{\boldsymbol{F}_{I}}, P_{L}\right)^{2}: \boldsymbol{F}_{I} \in \mathcal{A}_{I}^{y},\left\|S_{\boldsymbol{F}_{I}}\right\|_{\infty} \leq \delta\right\}$. The following proposition helps.

Proposition 6.2. As $\eta \rightarrow 0$ it holds $\mathrm{H}\left(Q_{\boldsymbol{F}_{I}}, P_{L}\right)^{2} \geq \frac{1}{8} \mathbb{E}_{L}\left[S_{\boldsymbol{F}_{I}}^{2}\right] e^{O(\eta)}$ for all $S_{\boldsymbol{F}_{I}}$ satisfying $\left\|S_{\boldsymbol{F}_{I}}\right\|_{\infty} \leq$ $\eta$. Then $\inf \left\{\mathrm{H}\left(Q_{\boldsymbol{F}_{I}}, P_{L}\right)^{2}: \boldsymbol{F}_{I} \in \mathcal{A}_{I}^{y},\left\|S_{\boldsymbol{F}_{I}}\right\|_{\infty} \leq \delta\right\} \geq \frac{y \mathcal{E}_{I}^{2}}{16}$ for $\delta$ small enough (but not depending on I nor on $y$ ).

For $\epsilon>0$ and any subset $A$ of a metric space equipped with metric $d$, we let $N(\epsilon, A, d)$ denote the $\epsilon$-covering number of $A$, i.e. the smallest number of balls of radius $\epsilon$ needed to cover $A$. if $d$ is induced by some norm $\|\cdot\|$, we write $N(\epsilon, A,\|\cdot\|)$. By Ghosal and van der Vaart (2007a, Corollary 1) our Proposition 6.2 implies that for all $D>0$, all $y \geq 1$, and all $n \geq 1$ there exists a test $\phi_{n, y}$ such that

$$
\mathbb{E}_{L}\left[\phi_{n, y}\right] \leq \frac{N\left(\frac{\sqrt{y} \mathcal{E}_{I}}{16}, \mathcal{P}_{I}^{y}, \mathrm{H}\right)}{D} \frac{e^{-\frac{y n \mathcal{E}_{I}^{2}}{256}}}{1-e^{-\frac{y n \mathcal{E}_{I}^{2}}{256}}}, \quad \sup _{\substack{\boldsymbol{F}_{I} \in \mathcal{A}_{I}^{y} \\\left\|S_{\boldsymbol{F}_{I}}\right\|_{\infty} \leq \delta}} \mathbb{E}_{\boldsymbol{F}_{I}}\left[1-\phi_{n, y}\right] \leq D e^{-\frac{y n \mathcal{E}_{I}^{2}}{256}}
$$

where $\mathbb{E}_{\boldsymbol{F}_{I}}$ is understood as the expectation under $Q_{\boldsymbol{F}_{I}}^{\otimes n}$, and where $\mathcal{P}_{I}^{y}:=\left\{q_{\boldsymbol{F}_{I}}: \boldsymbol{F}_{I} \in \mathcal{A}_{I}^{y},\left\|S_{\boldsymbol{F}_{I}}\right\|_{\infty} \leq\right.$ $\delta\}$. Using the estimate of Lemma 6.1 we find that $\mathbb{E}_{L}\left[\Pi\left(\mathscr{C}_{*} \cap \mathscr{S}_{I}^{y} \mid \mathbf{X}_{n}\right) \mathbf{1}_{\Omega_{t}}\right]$ is bounded by

$$
\begin{aligned}
\mathbb{E}_{L}\left[\phi_{n, y} \Pi\left(\mathscr{C}_{*} \cap \mathscr{S}_{I}^{y} \mid \mathbf{X}_{n}\right)\right]+\mathbb{E}_{L}\left[\left(1-\phi_{n, y}\right) \Pi\left(\mathscr{C}_{*} \cap \mathscr{S}_{I}^{y} \mid \mathbf{X}_{n}\right) \mathbf{1}_{\Omega_{t}}\right] \\
\leq \mathbb{E}_{L}\left[\phi_{n, y}\right]+2 e^{2 \delta t+c_{0} \delta y n \mathcal{E}_{I}^{2}} \int_{\mathcal{A}_{I}^{y} \cap\left\{\left\|S_{\boldsymbol{F}_{I}}\right\|_{\infty} \leq \eta\right\}} \mathbb{E}_{\boldsymbol{F}_{I}}\left[1-\phi_{n, y}\right] \frac{\Pi\left(\mathrm{d} \boldsymbol{F}_{I}\right)}{\Pi\left(\tilde{\mathcal{A}}_{I}\right)} \\
\leq \frac{N\left(\frac{\sqrt{y} \mathcal{E}_{I}}{16}, \mathcal{P}_{I}^{y}, \mathrm{H}\right)}{D} \frac{e^{-\frac{y n \mathcal{E}_{I}^{2}}{256}}}{1-e^{-\frac{y n \mathcal{E}_{I}^{2}}{256}}}+2 D e^{2 \delta t+c_{0} \delta y n \mathcal{E}_{I}^{2}-\frac{y n \mathcal{E}_{I}^{2}}{256}} \frac{\Pi\left(\mathcal{A}_{I}^{y}\right)}{\Pi\left(\tilde{\mathcal{A}}_{I}\right)}
\end{aligned}
$$

The previous display is true for any $D>0$ and thus we can optimize over $D$, which is also known as the square-root trick (Lijoi, Prünster and Walker, 2005; Walker, Lijoi and Prünster, 2007). Doing so gives the bound,

$$
\mathbb{E}_{L}\left[\Pi\left(\mathscr{C}_{*} \cap \mathscr{S}_{I}^{y} \mid \mathbf{X}_{n}\right) \mathbf{1}_{\Omega_{t}}\right] \leq\left\{\frac{8 e^{2 \delta t+c_{0} \delta y n \mathcal{E}_{I}^{2}} N\left(\frac{\sqrt{y} \mathcal{E}_{I}}{16}, \mathcal{P}_{I}^{y}, \mathrm{H}\right)}{1-e^{-y n \mathcal{E}_{I}^{2} / 256}} \frac{\Pi\left(\mathcal{A}_{I}^{y}\right)}{\Pi\left(\tilde{\mathcal{A}}_{I}\right)}\right\}^{1 / 2} e^{-\frac{y n \mathcal{E}_{I}^{2}}{256}}
$$

To obtain the bound in the statement of the lemma, it is enough to prove that $\sup _{y \geq 1} N\left(\frac{\sqrt{y} \mathcal{E}_{I}}{16}, \mathcal{P}_{I}^{y}, \mathrm{H}\right) \leq$ $\exp \left(c_{1} 2^{J_{0}}|I|\right)$. The following lemma helps. 
Proposition 6.3. There exists $\delta_{0}>0$ such that for all $\delta \leq \delta_{0}$, all $I \subseteq \mathbb{Z}_{+}$, and all $\boldsymbol{F}_{I}, \boldsymbol{F}_{I}^{\prime} \in \mathcal{A}_{I} \cap$ $\left\{\left\|S_{\boldsymbol{F}_{I}}\right\|_{\infty} \leq \delta\right\}$ it holds $\mathrm{H}\left(Q_{\boldsymbol{F}_{I}}, Q_{\boldsymbol{F}_{I}^{\prime}}\right)^{2} \leq \frac{1}{2} \mathbb{E}_{L}\left[\left(S_{\boldsymbol{F}_{I}}-S_{\boldsymbol{F}_{I}^{\prime}}\right)^{2}\right]$.

Observe that for $\boldsymbol{F}_{I}, \boldsymbol{F}_{I}^{\prime} \in \mathcal{A}_{I}$ we have $S_{\boldsymbol{F}_{I}}-S_{\boldsymbol{F}_{I}^{\prime}}=\sum_{m \in I}\left(F_{m}-F_{m}^{\prime}\right)$, where by construction each $F_{m}-F_{m}^{\prime}$ is in $\mathcal{F}_{m}$. Then, by Proposition 7.1-(4), and then by Proposition 7.1-(3),

$$
\mathbb{E}_{L}\left[\left(S_{\boldsymbol{F}_{I}}-S_{\boldsymbol{F}_{I}^{\prime}}\right)^{2}\right] \lesssim \sum_{m \in I}\left\|F_{m}-F_{m}^{\prime}\right\|_{2}^{2} \lesssim \sum_{m \in I} \sum_{v \in B_{m}}\left\langle F_{m}-F_{m}^{\prime}, \varphi_{v}\right\rangle^{2}
$$

On the other hand, for all $\boldsymbol{F}_{I} \in \mathcal{A}_{I}^{y}$, we have by Proposition 7.1 that,

$$
\sum_{m \in I} \sum_{v \in B_{m}}\left\langle F_{m}, \varphi_{v}\right\rangle^{2} \lesssim \sum_{m \in I}\left\|F_{m}\right\|_{2}^{2} \lesssim \mathbb{E}_{L}\left[S_{\boldsymbol{F}_{I}}^{2}\right] \lesssim y \mathcal{E}_{I}^{2}
$$

By equations (6.5) and (6.6) and Proposition 6.3, we find that $N\left(\frac{\sqrt{y} \mathcal{E}_{I}}{16}, \mathcal{P}_{I}^{y}, \mathrm{H}\right)$ is no more than the covering number of a ball of radius $\lesssim \sqrt{y} \mathcal{E}_{I}$ with balls of radius $\asymp \sqrt{y} \mathcal{E}_{I}$ in $\mathbb{R}^{p}$ equipped with the euclidean distance, with $p=\sum_{m \in I}\left|B_{m}\right| \leq\left|B_{0}\right| \cdot|I|$. By Pollard (1990, Lemma 4.1), this implies that there is a universal $K>0$ such that

$$
N\left(\frac{\sqrt{y} \mathcal{E}_{I}}{16}, \mathcal{P}_{I}^{y}, \mathrm{H}\right) \leq \max \left\{1,\left(\frac{3 K \sqrt{y} \mathcal{E}_{I}}{\sqrt{y} \mathcal{E}_{I}}\right)^{p}\right\} \leq \max \left\{1,(3 K)^{p}\right\} .
$$

Finally, by construction it is true that $\left|B_{0}\right| \lesssim 2^{J_{0}}$.

Proof of Lemma 6.3. We use the fact that for all $u, v \geq 0$ we have $\min \{u, v\}=\inf _{\beta \in(0,1)}\left\{u^{1-\beta} v^{\beta}\right\}$. Then, combining the bounds of Lemma 6.2 with the bound of equation (6.2), we get that for any $\beta \in(0,1)$ and $y \geq 1$

$$
\begin{aligned}
\frac{\mathbb{E}_{L}\left[\Pi\left(\mathscr{C}_{*} \cap \mathscr{S}_{I}^{y} \mid \mathbf{X}_{n}\right) \mathbf{1}_{\Omega_{t}}\right]}{\sqrt{8} e^{2 \delta t}} & \leq \min \left\{e^{c_{0} \delta y n \mathcal{E}_{I}^{2}} \frac{\Pi\left(\mathcal{A}_{I}^{y}\right)}{\Pi\left(\tilde{\mathcal{A}}_{I}\right)},\left(\frac{\exp \left(-\frac{y n \mathcal{E}_{I}^{2}}{256}+c_{1} 2^{J_{0}}|I|\right)}{1-e^{-y n \mathcal{E}_{I}^{2} / 256}} \frac{\Pi\left(\mathcal{A}_{I}^{y}\right)}{\Pi\left(\tilde{\mathcal{A}}_{I}\right)}\right)^{1 / 2}\right\} \\
& \leq \frac{\exp \left\{-y n \mathcal{E}_{I}^{2}\left(\frac{\beta}{512}+c_{0} \delta \beta-c_{0} \delta\right)+\frac{\beta c_{1} 2^{J_{0}|I|}}{2}\right\}}{\left(1-e^{-n \mathcal{E}_{I}^{2} / 256}\right)^{\beta / 2}}\left\{\frac{\Pi\left(\mathcal{A}_{I}^{y}\right)}{\Pi\left(\tilde{\mathcal{A}}_{I}\right)}\right\}^{1-\beta / 2} .
\end{aligned}
$$

Hence, for any $\frac{512 \cdot c_{0} \delta}{1+512 \cdot c_{0} \delta}<\beta \leq 1$, writing $\mu:=\frac{\beta}{512}+c_{0} \delta \beta-c_{0} \delta>0$ for simplicity, by Hölder's inequality,

$$
\begin{aligned}
\sum_{y \geq 1} e^{-y \mu n \mathcal{E}_{I}^{2}}\left\{\frac{\Pi\left(\mathcal{A}_{I}^{y}\right)}{\Pi\left(\tilde{\mathcal{A}}_{I}\right)}\right\}^{1-\beta / 2} & \leq\left\{\sum_{y \geq 1} e^{-2 y \mu n \mathcal{E}_{I}^{2} / \beta}\right\}^{\beta / 2}\left\{\sum_{y \geq 1} \frac{\Pi\left(\mathcal{A}_{I}^{y}\right)}{\Pi\left(\tilde{\mathcal{A}}_{I}\right)}\right\}^{1-\beta / 2} \\
& =\frac{e^{-\mu n \mathcal{E}_{I}^{2}}}{\left(1-e^{-2 y \mu n \mathcal{E}_{I}^{2} / \beta}\right)^{\beta / 2}}\left\{\frac{\Pi\left(\mathcal{A}_{I}\right)}{\Pi\left(\tilde{\mathcal{A}}_{I}\right)}\right\}^{1-\beta / 2}
\end{aligned}
$$

where the second line follows since $\left(\mathcal{A}_{I}^{y}\right)_{y \geq 1}$ is a partition of $\mathcal{A}_{I}$. Therefore,

$$
\sum_{y \geq 1} \frac{\mathbb{E}_{L}\left[\Pi\left(\mathscr{C}_{*} \cap \mathscr{S}_{I}^{y} \mid \mathbf{X}_{n}\right) \mathbf{1}_{\Omega_{t}}\right]}{\sqrt{8} e^{2 \delta t}} \leq \frac{\exp \left\{-\mu n \mathcal{E}_{I}^{2}+\frac{\beta c_{1} 2^{J} 0|I|}{2}\right\}}{\left(1-e^{-n \mathcal{E}_{I}^{2} / 256}\right)^{\beta / 2}\left(1-e^{-2 \mu n \mathcal{E}_{I}^{2} / \beta}\right)^{\beta / 2}}\left\{\frac{\Pi\left(\mathcal{A}_{I}\right)}{\Pi\left(\tilde{\mathcal{A}}_{I}\right)}\right\}^{1-\beta / 2} .
$$


By taking $\alpha=\beta / 2$ and $\beta>\frac{1024 \cdot C \delta}{1+512 \cdot C \delta}$ we have that $\mu>\frac{1}{2}\left(\frac{1}{512}+C \delta\right) \beta$, whence the conclusion.

\subsection{Control of the covariance terms}

The major difficulty in establishing the Theorem 3.1 is to prove estimates on the covariance terms $\mathrm{C}\left(S_{\boldsymbol{F}_{I}}, S_{\overline{\boldsymbol{F}}_{I}}\right)$ that are sharp enough. The estimate used in the proof of Theorem 3.1 are established in the Corollaries 6.1 and 6.2 below, which are consequences of the next lemma. The proof of the Lemma 6.4 is quite long and is deferred to Section S5.2.

Lemma 6.4. Suppose Assumption 1 is satisfied with constants $K_{0}, K_{1}, K_{2}>0$ sufficiently large. Then, there is a constant $C>0$ such that for all $1<\delta \leq 1$, for all $I \subseteq \mathbb{Z}_{+}$, for all $\left\|S_{\boldsymbol{F}_{I}}\right\|_{\infty} \leq \delta$, and for all $\overline{\boldsymbol{F}}_{I} \in \mathcal{N}_{I}$, if $s>1 / 2$

$$
\begin{aligned}
\left|\mathrm{C}\left(S_{\boldsymbol{F}_{I}}, S_{\overline{\boldsymbol{F}}_{I}}\right)\right| \leq & C \delta \mathbb{E}_{L}\left[S_{\boldsymbol{F}_{I}}^{2}\right]+\delta \Gamma \sqrt{\frac{\log (n)}{n}}\left\|F_{0}\right\|_{2} \mathbf{1}_{0 \in I} \\
& +\delta \Gamma \xi^{-\mathbf{1}_{0 \in I}}\left\{\sum_{m \in I}\left\|F_{m}\right\|_{2}^{2} \mathbf{1}_{J_{m} \leq j_{n}}\right\}^{1 / 2} \sqrt{\frac{\log (n)}{n}} \\
& +\frac{\delta}{\sqrt{n}}\left\{\sum_{m \in I}\left\|F_{m}\right\|_{2}^{2} \mathbf{1}_{J_{m}>j_{n}}\right\}^{1 / 2}
\end{aligned}
$$

and if $0<s \leq 1 / 2$,

$$
\begin{aligned}
\left|\mathrm{C}\left(S_{\boldsymbol{F}_{I}}, S_{\overline{\boldsymbol{F}}_{I}}\right)\right| \leq & C \delta \mathbb{E}_{L}\left[S_{\boldsymbol{F}_{I}}^{2}\right]+\delta \Gamma 2^{-J_{0} / 2} \varepsilon_{n}^{*}(s)\left\|F_{0}\right\|_{2} \mathbf{1}_{0 \in I} \\
& +\delta\left\{\sum_{m \in I}\left\|F_{m}\right\|_{2}^{2} \mathbf{1}_{J_{m} \leq j_{n}}\right\}^{1 / 2}\left\{\Gamma^{2} \xi^{-2 \mathbf{1}_{0 \in I}} \varepsilon_{n}^{*}(s)^{2} \sum_{m \in I} 2^{-J_{m}} \mathbf{1}_{J_{m} \leq j_{n}}\right\}^{1 / 2} \\
& +\delta\left\{\sum_{m \in I}\left\|F_{m}\right\|_{2}^{2} \mathbf{1}_{J_{m}>j_{n}}\right\}^{1 / 2}\left\{\gamma \sum_{m \in I} 2^{-J_{m}(2 s+1)} \mathbf{1}_{J_{m}>j_{n}}\right\}^{1 / 2} .
\end{aligned}
$$

Corollary 6.1. Suppose Assumption 1 is satisfied with constants $K_{0}, K_{1}, K_{2}>0$ sufficiently large. Then, there is a constant $C>0$ such that for all $1<\delta \leq 1$, for all $I \subseteq \mathbb{Z}_{+}$, for all $y \geq 1$, for all $\boldsymbol{F}_{I} \in \mathcal{A}_{I}^{y}$, and for all $\overline{\boldsymbol{F}}_{I} \in \mathcal{N}_{I}$

$$
\mathrm{C}\left(S_{\boldsymbol{F}_{I}}, S_{\overline{\boldsymbol{F}}_{I}}\right) \geq-C \delta y \mathcal{E}_{I}^{2}-\frac{\delta}{n} .
$$

Corollary 6.2. Suppose Assumption 1 is satisfied with constants $K_{0}, K_{1}, K_{2}>0$ sufficiently large. Then, there is a constant $C>0$ such that for all $1<\delta \leq 1$, for all $I \subseteq \mathbb{Z}_{+}$,

$$
\sup \left\{\mathrm{C}\left(S_{\boldsymbol{F}_{I}}, S_{\overline{\boldsymbol{F}}_{I}}\right): \boldsymbol{F}_{I} \in \tilde{\mathcal{A}}_{I}, \overline{\boldsymbol{F}}_{I} \in \mathcal{N}_{I}\right\} \leq C \delta^{2} \mathcal{E}_{I}^{2}
$$




\subsection{Proofs of Propositions 6.1 to 6.3}

Proof of Proposition 6.1. The proof is an adaptation of the classical Ghosal, Ghosh and van der Vaart (2000, Lemma 8.1). The first step if to remark that by Jensen's inequality applied to the logarithm

$$
\begin{aligned}
\log \int_{\tilde{\mathcal{A}}_{I}} e^{n \mathbb{P}_{n} \Phi\left(S_{\boldsymbol{F}_{I}}\right)} \Pi\left(\mathrm{d} \boldsymbol{F}_{I}\right) \geq & \sum_{i=1}^{n} \int_{\tilde{\mathcal{A}}_{I}}\left(\Phi S_{\boldsymbol{F}_{I}}\left(X_{i}\right)-\mathbb{E}_{L}\left[\Phi S_{\boldsymbol{F}_{I}}\right]\right) \Pi\left(\mathrm{d} \boldsymbol{F}_{I}\right) \\
& +n \int_{\tilde{\mathcal{A}}_{I}} \mathbb{E}_{L}\left[\Phi S_{\boldsymbol{F}_{I}}\right] \Pi\left(\mathrm{d} \boldsymbol{F}_{I}\right) .
\end{aligned}
$$

For all $\boldsymbol{F}_{I} \in \tilde{\mathcal{A}}_{I}$, since $\mathbb{E}_{L}\left[S_{\boldsymbol{F}_{I}}\right]=0$, we have whenever $0<\delta \leq \log (2)$,

$$
\begin{aligned}
\mathbb{E}_{L}\left[\Phi S_{\boldsymbol{F}_{I}}\right] & =-\log \mathbb{E}_{L}\left[e^{\left.S_{\boldsymbol{F}_{I}}\right]}\right. \\
& \geq-\log \mathbb{E}_{L}\left[1+S_{\boldsymbol{F}_{I}}+\frac{1}{2} S_{\boldsymbol{F}_{I}}^{2} e^{\left\|S_{\boldsymbol{F}_{I}}\right\|_{\infty}}\right] \\
& \geq-\log \left(1+\mathbb{E}_{L}\left[S_{\boldsymbol{F}_{I}}^{2}\right]\right) .
\end{aligned}
$$

By definition $\mathbb{E}_{L}\left[S_{\boldsymbol{F}_{I}}^{2}\right] \leq \delta^{2} \mathcal{E}_{I}^{2}$ whenever $\boldsymbol{F}_{I} \in \tilde{\mathcal{A}}_{I}$. That is $\mathbb{E}_{L}\left[\Phi S_{\boldsymbol{F}_{I}}\right] \geq-\mathbb{E}_{L}\left[S_{\boldsymbol{F}_{I}}^{2}\right] \geq-\delta^{2} \mathcal{E}_{I}^{2}$ for any $\boldsymbol{F}_{I} \in \tilde{\mathcal{A}}_{I}$. Hence,

$$
\log \int_{\tilde{\mathcal{A}}_{I}} e^{n \mathbb{P}_{n} \Phi\left(S_{\boldsymbol{F}_{I}}\right)} \Pi\left(\mathrm{d} \boldsymbol{F}_{I}\right) \geq \sum_{i=1}^{n} \int_{\tilde{\mathcal{A}}_{I}}\left(\Phi S_{\boldsymbol{F}_{I}}\left(X_{i}\right)-\mathbb{E}_{L}\left[\Phi S_{\boldsymbol{F}_{I}}\right]\right) \Pi\left(\mathrm{d} \boldsymbol{F}_{I}\right)-n \delta^{2} \mathcal{E}_{I}^{2} .
$$

Now we define the random variables $Z_{i}:=\int_{\tilde{\mathcal{A}}_{I}}\left(\Phi S_{\boldsymbol{F}_{I}}\left(X_{i}\right)-\mathbb{E}_{L}\left[\Phi S_{\boldsymbol{F}_{I}}\right]\right) \Pi\left(\mathrm{d} \boldsymbol{F}_{I}\right)$. Observe that $\mathbb{E}_{L}\left[Z_{i}\right]=0$, and $\Phi S_{\boldsymbol{F}_{I}}-\mathbb{E}_{L}\left[\Phi S_{\boldsymbol{F}_{I}}\right]=S_{\boldsymbol{F}_{I}}$, so we have $\left|Z_{i}\right| \leq \delta$ because $\Pi$ is a probability measure. Further, by an application of Jensen's inequality and Fubini's theorem

$$
\begin{aligned}
\mathbb{E}_{L}\left[Z_{i}^{2}\right] & =\mathbb{E}_{L}\left[\left(\int_{\tilde{\mathcal{A}}_{I}} S_{\boldsymbol{F}_{I}}\left(X_{i}\right) \Pi\left(\mathrm{d} \boldsymbol{F}_{I}\right)\right)^{2}\right] \\
& \leq \mathbb{E}_{L}\left[\int_{\tilde{\mathcal{A}}_{I}} S_{\boldsymbol{F}_{I}}\left(X_{i}\right)^{2} \Pi\left(\mathrm{d} \boldsymbol{F}_{I}\right)\right] \\
& =\int_{\tilde{\mathcal{A}}_{I}} \mathbb{E}_{L}\left[S_{\boldsymbol{F}_{I}}^{2}\right] \Pi\left(\mathrm{d} \boldsymbol{F}_{I}\right) .
\end{aligned}
$$

Therefore $\mathbb{E}_{L}\left[Z_{i}^{2}\right] \leq \delta^{2} \mathcal{E}_{I}^{2}$, because of the definition of $\tilde{\mathcal{A}}_{I}$, and because $\Pi$ is a probability measure. By the equation (6.7), the probability of $\Omega_{t}^{c}$ is no more than the probability of having $\sum_{i=1}^{n} Z_{i} \leq-\delta t-$ $\sqrt{2 n \delta^{2} \mathcal{E}_{I}^{2} t}$. The conclusion of the proposition then follows by Bernstein's inequality (Boucheron, Lugosi and Massart, 2013, Theorem 2.10).

Proof of Proposition 6.2. Observe that by definition $q_{\boldsymbol{F}_{I}}(x)=p_{L}(x) e^{\Phi\left(S_{\boldsymbol{F}_{I}}\right)}$. Also we have $\mathbb{E}_{L}\left[S_{\boldsymbol{F}_{I}}\right]=$ 0 and thus $\Phi\left(S_{\boldsymbol{F}_{I}}\right)=S_{\boldsymbol{F}_{I}}-\log \mathbb{E}_{L}\left[e^{\left.S_{\boldsymbol{F}_{I}}\right]}\right.$. We lower bound $\mathrm{H}\left(Q_{\boldsymbol{F}_{I}}, P_{L}\right)$ by obtaining an upper bound on the Hellinger affinity $\mathrm{R}\left(Q_{\boldsymbol{F}_{I}}, P_{L}\right):=\int_{[0,1]} \sqrt{q_{\boldsymbol{F}_{I}} p_{L}}$ and using that $\mathrm{H}\left(Q_{\boldsymbol{F}_{I}}, P_{L}\right)^{2}=1-$

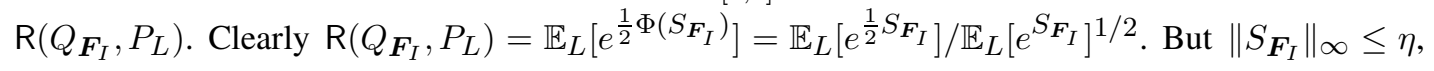




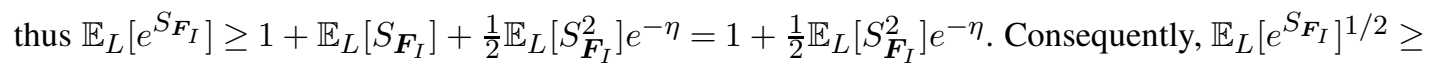

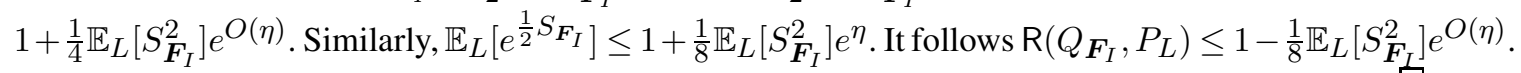

Proof of Proposition 6.3. Remark that $\mathbb{E}_{L}\left[S_{\boldsymbol{F}_{I}}\right]=0$, so $\Phi\left(S_{\boldsymbol{F}_{I}}\right)=S_{\boldsymbol{F}_{I}}-\log \mathbb{E}_{L}\left[e^{S_{\boldsymbol{F}_{I}}}\right]$, and similarly for $\Phi\left(S_{\boldsymbol{F}_{I}^{\prime}}\right)$. Since $\left\|S_{\boldsymbol{F}_{I}}\right\|_{\infty} \leq \delta$ then $\left\|\Phi\left(S_{\boldsymbol{F}_{I}}\right)\right\|_{\infty} \leq 2 \delta$, similarly for $S_{\boldsymbol{F}_{I}^{\prime}}$. By a Taylor expansion there is $u \in\left(\Phi\left(S_{\boldsymbol{F}_{I}}\right), \Phi\left(S_{\boldsymbol{F}_{I}^{\prime}}\right)\right)$, and hence $|u| \leq 2 \delta$, such that $e^{\frac{1}{2} \Phi\left(S_{\boldsymbol{F}_{I}}\right)}=e^{\frac{1}{2} \Phi\left(S_{\boldsymbol{F}_{I}^{\prime}}\right)}+\frac{1}{2}\left(\Phi\left(S_{\boldsymbol{F}_{I}}\right)-\right.$ $\Phi\left(S_{\boldsymbol{F}_{I}^{\prime}}\right) e^{u}$. That is, $\left(e^{\frac{1}{2} \Phi\left(S_{\boldsymbol{F}_{I}}\right)}-e^{\frac{1}{2} \Phi\left(S_{\boldsymbol{F}_{I}^{\prime}}\right)}\right)^{2} \leq \frac{1}{4} e^{4 \delta}\left(\Phi\left(S_{\boldsymbol{F}_{I}}\right)-\Phi\left(S_{\boldsymbol{F}_{I}^{\prime}}\right)\right)^{2}$. Then we can bound the Hellinger distance as follows.

$$
\begin{aligned}
\mathrm{H}\left(Q_{\boldsymbol{F}_{I}}, Q_{\boldsymbol{F}_{I}^{\prime}}\right)^{2} & =\frac{1}{2} \int\left(\sqrt{p_{L} e^{\Phi\left(S_{\boldsymbol{F}_{I}}\right)}}-\sqrt{p_{L} e^{\Phi\left(S_{\boldsymbol{F}_{I}^{\prime}}\right)}}\right)^{2} \\
& =\frac{1}{2} \mathbb{E}_{L}\left[\left(e^{\frac{1}{2} \Phi\left(S_{\boldsymbol{F}_{I}}\right)}-e^{\frac{1}{2} \Phi\left(S_{\boldsymbol{F}_{I}^{\prime}}\right)}\right)^{2}\right] \\
& \leq \frac{1}{8} e^{4 \delta_{\mathbb{E}_{L}}\left[\left(\Phi\left(S_{\boldsymbol{F}_{I}}\right)-\Phi\left(S_{\boldsymbol{F}_{I}^{\prime}}\right)\right)^{2}\right] .}
\end{aligned}
$$

Expanding the square in the last equation and using that $\mathbb{E}_{L}\left[S_{\boldsymbol{F}_{I}}\right]=\mathbb{E}_{L}\left[S_{\boldsymbol{F}_{I}^{\prime}}\right]=0$, we find that

$$
\mathrm{H}\left(Q_{\boldsymbol{F}_{I}}, Q_{\boldsymbol{F}_{I}^{\prime}}\right)^{2} \leq \frac{1}{8} e^{4 \delta} \mathbb{E}_{L}\left[\left(S_{\boldsymbol{F}_{I}}-S_{\boldsymbol{F}_{I}^{\prime}}\right)^{2}\right]+\frac{1}{8} e^{4 \delta} \log ^{2} \frac{\mathbb{E}_{L}\left[e^{S_{\boldsymbol{F}_{I}}}\right]}{\mathbb{E}_{L}\left[e^{\left.S_{\boldsymbol{F}_{I}^{\prime}}\right]} .\right.}
$$

Now remark that $\mathbb{E}_{L}\left[e^{S_{\boldsymbol{F}_{I}}}\right]=\mathbb{E}_{L}\left[e^{S_{\boldsymbol{F}_{I}^{\prime}}} e^{S_{\boldsymbol{F}_{I}}-S_{\boldsymbol{F}_{I}^{\prime}}}\right]=\mathbb{E}_{L}\left[e^{S_{\boldsymbol{F}_{I}^{\prime}}}\right]+\mathbb{E}_{L}\left[e^{S_{\boldsymbol{F}_{I}^{\prime}}}\left(e^{S_{\boldsymbol{F}_{I}}-S_{\boldsymbol{F}_{I}^{\prime}}}-1\right)\right]$, and hence

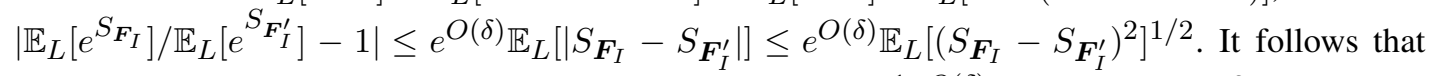
the second term of the rhs of equation (6.8) is bounded by $\frac{1}{8} e^{O(\delta)} \mathbb{E}_{L}\left[\left(S_{\boldsymbol{F}_{I}}-S_{\boldsymbol{F}_{I}^{\prime}}\right)^{2}\right]$, and hence $\mathrm{H}\left(Q_{\boldsymbol{F}_{I}}, Q_{\boldsymbol{F}_{I}^{\prime}}\right) \leq \frac{1}{4} e^{O(\delta)} \mathbb{E}_{L}\left[\left(S_{\boldsymbol{F}_{I}}-S_{\boldsymbol{F}_{I}^{\prime}}\right)^{2}\right]$.

\subsection{Proofs of Corollaries 6.1 and 6.2}

Proof of Corollary 6.1: the case where $s>1 / 2$. By construction, if $m \in I$ and $\boldsymbol{F} \in \mathcal{A}_{I}$, then $\left\|F_{m}\right\|_{2} \geq$ $H_{I}(m)$. Thus, if $I \cap\left\{m: J_{m} \leq j_{n}\right\} \neq \varnothing$, for all $\boldsymbol{F} \in \mathcal{A}_{I}$,

$$
\left(\Gamma \xi^{-\mathbf{1}_{0 \in I}}\right)^{2} \frac{\log (n)}{n} \leq \sum_{m \in I} H_{I}(m)^{2} \mathbf{1}_{J_{m} \leq j_{n}} \leq \sum_{m \in I}\left\|F_{m}\right\|_{2}^{2} \mathbf{1}_{J_{m} \leq j_{n}} .
$$

Also if $0 \in I$, then $\Gamma^{2} \log (n) / n \leq\left\|F_{0}\right\|_{2}^{2}$. Therefore Lemma 6.4 and Young's inequality imply

$$
\begin{aligned}
\left|\mathrm{C}\left(S_{\boldsymbol{F}_{I}}, S_{\overline{\boldsymbol{F}}_{I}}\right)\right| \leq C \delta \mathbb{E}_{L}\left[S_{\boldsymbol{F}_{I}}^{2}\right]+\delta\left\|F_{0}\right\|_{2}^{2} \mathbf{1}_{0 \in I}+\delta \sum_{m \in I}\left\|F_{m}\right\|_{2}^{2} \mathbf{1}_{J_{m} \leq j_{n}} & \\
& +\frac{\delta}{2} \sum_{m \in I}\left\|F_{m}\right\|_{2}^{2} \mathbf{1}_{J_{m}>j_{n}}+\frac{\delta}{2 n} .
\end{aligned}
$$

The conclusion follows since $\sum_{m \in I}\left\|F_{m}\right\|_{2}^{2} \asymp \mathbb{E}_{L}\left[S_{\boldsymbol{F}_{I}}^{2}\right]$ by Proposition 7.1-(4). 
Proof of Corollary 6.1: the case where $0<s \leq 1 / 2$. By construction, if $m \in I$ and $\boldsymbol{F} \in \mathcal{A}_{I}$, then $\left\|F_{m}\right\|_{2} \geq H_{I}(m)$. Thus, if $I \cap\left\{m: J_{m} \leq j_{n}\right\} \neq \varnothing$, for all $\boldsymbol{F} \in \mathcal{A}_{I}$,

$$
\left(\Gamma \xi^{-\mathbf{1}_{0 \in I}}\right)^{2} \varepsilon_{n}^{*}(s)^{2} \sum_{m \in I} 2^{-J_{m}} \mathbf{1}_{J_{m} \leq j_{n}} \leq \sum_{m \in I}\left\|F_{m}\right\|_{2}^{2} \mathbf{1}_{J_{m} \leq j_{n}} .
$$

Similarly if $I \cap\left\{m: J_{m}>j_{n}\right\} \neq \varnothing$, we have by construction for all $\boldsymbol{F} \in \mathcal{A}_{I}$

$$
\gamma^{2} \sum_{m \in I} 2^{-J_{m}(2 s+1)} \mathbf{1}_{J_{m}>j_{n}} \leq \sum_{m \in I}\left\|F_{m}\right\|_{2}^{2} \mathbf{1}_{J_{m}>j_{n}} .
$$

Also of $0 \in I$, then $\Gamma^{2} 2^{-J_{0}} \varepsilon_{n}^{*}(s)^{2} \leq\left\|F_{0}\right\|_{2}^{2}$. Therefore Lemma 6.4 implies

$$
\left|\mathrm{C}\left(S_{\boldsymbol{F}_{I}}, S_{\overline{\boldsymbol{F}}_{I}}\right)\right| \leq C \delta \mathbb{E}_{L}\left[S_{\boldsymbol{F}_{I}}^{2}\right]+\delta\left\|F_{0}\right\|_{2}^{2} \mathbf{1}_{0 \in I}+\delta \sum_{m \in I}\left\|F_{m}\right\|_{2}^{2}
$$

The conclusion follows since $\sum_{m \in I}\left\|F_{m}\right\|_{2}^{2} \asymp \mathbb{E}_{L}\left[S_{\boldsymbol{F}_{I}}^{2}\right]$ by Proposition 7.1-(4).

Proof of Corollary 6.2. The proof follows immediately from Lemma 6.4, from the fact that $\sum_{m \in I}\left\|F_{m}\right\|_{2}^{2} \asymp$ $\mathbb{E}_{L}\left[S_{\boldsymbol{F}_{I}}^{2}\right]$ by Proposition 7.1-(4), and from the definition of $\tilde{\mathcal{A}}_{I}$.

\section{Auxiliary results and remaining proofs}

\subsection{Relations between norms}

In many places we need to relate norm of various functions. In this section we collect the propositions that serve this purpose.

Proposition 7.1. Let $J_{0}$ be chosen large enough. Then the following are true.

1. For all $m \in \mathbb{Z}_{+}$and all $F \in \mathcal{F}_{m}, F=\sum_{(j, k) \in B_{m}}\left\langle F, \varphi_{j, k}\right\rangle\left(\varphi_{j, k}-\mathbb{E}_{L}\left[\varphi_{j, k}\right]\right)$;

2. For all $m \in \mathbb{Z}_{+}$, all $F \in \mathcal{F}_{m}$, and all $(j, k) \notin B_{0} \cup B_{m} \Longrightarrow\left\langle F, \varphi_{j, k}\right\rangle=0$.

3. There exist constants $C_{1}, C_{2}>0$ such that for all $m \in \mathbb{Z}_{+}$and all $F \in \mathcal{F}_{m}, C_{1} \sum_{v \in B_{m}}\left\langle F, \varphi_{v}\right\rangle^{2} \leq$ $\|F\|_{2}^{2} \leq C_{2} \sum_{v \in B_{m}}\left\langle F, \varphi_{v}\right\rangle^{2}$.

4. There exist constants $C_{1}, C_{2}>0$ such that for all $J \subseteq \mathbb{Z}_{+}$, for all collections $\left\{F_{m} \in \mathcal{F}_{m}\right.$ : $m \in J\}, C_{1} \sum_{m \in J}\left\|F_{m}\right\|_{2}^{2} \leq \mathbb{E}_{L}\left[\left(\sum_{m \in J} F_{m}\right)^{2}\right] \leq C_{2} \sum_{m \in J}\left\|F_{m}\right\|_{2}^{2}$.

5. There exist constants $C_{1}, C_{2}>0$ such that $\sup _{x} \sum_{k}\left|\varphi_{j, k}(x)\right| \leq C_{1} 2^{j / 2}$ for all $j \geq 0$, and $\sum_{k}\left|\mathbb{E}_{L}\left[\varphi_{j, k}\right]\right| \leq C_{2} 2^{j / 2}$ for all $j \geq 0$. Consequently, $\sup _{x} \sum_{k}\left|\varphi_{j, k}(x)-\mathbb{E}_{L}\left[\varphi_{j, k}\right]\right| \leq 2 \max \left\{C_{1}, C_{2}\right\}^{j / 2}$ for all $j \geq 0$;

Proposition 7.2. Suppose Assumption 1 is valid. There exists a universal constant $C>0$ such that for all $I \subseteq \mathbb{Z}_{+}$,

$$
\mathcal{E}_{I}^{2} \geq C\left(\frac{\Gamma^{2} \log (n)}{n} \sum_{m \in I} \xi^{-2 \mathbf{1}_{0 \in I} \mathbf{1}_{m \neq 0}} \mathbf{1}_{J_{m} \leq j_{n}}+\gamma^{2} \sum_{m \in I} 2^{-J_{m}(2 s+1)} \mathbf{1}_{J_{m}>j_{n}}\right) .
$$




\subsection{Proofs of the lemmas used in the guidelines of Section 3 and proof of the Corollary 3.1}

Proof of Lemma 3.2. Write $g=\sum_{m>0} F_{m}$ for simplicity. Then, remark that $\mathbb{E}_{L}[g]=0$, and thus $\mathbb{E}_{L}\left[e^{g}\right] \geq \mathbb{E}_{L}\left[1+g+\frac{1}{2} g^{2} e^{-\|g\|_{\infty}}\right]=1+\frac{1}{2} \mathbb{E}_{L}\left[g^{2}\right] e^{-\|g\|_{\infty}} \geq 1$, and with the same argument $1 \leq$ $\mathbb{E}_{L}\left[e^{g}\right] \leq 1+\frac{1}{2} \mathbb{E}_{L}\left[g^{2}\right] e^{\|g\|_{\infty}}$. It follows from equation (3.1) that $\left|L_{\boldsymbol{F}}-L\right| \leq|g|+\left|\log \mathbb{E}_{L}\left[e^{g}\right]\right|=$ $|g|+\log \mathbb{E}_{L}\left[e^{g}\right] \leq|g|+\frac{1}{2} \mathbb{E}_{L}\left[g^{2}\right] e^{\|g\|_{\infty}}$. Since $\mathbb{E}_{L}\left[g^{2}\right] \leq\|g\|_{\infty}^{2}$,

$$
\begin{aligned}
\left\|L_{\boldsymbol{F}}-L\right\|_{\infty} & \leq\left\|\sum_{m \geq 0} F_{m}\right\|_{\infty}\left(1+\frac{1}{2}\left\|\sum_{m \geq 0} F_{m}\right\|_{\infty} e^{\left\|\sum_{m \geq 0} F_{m}\right\|_{\infty}}\right) \\
& \lesssim\left\|\sum_{m \geq 0} F_{m}\right\|_{\infty},
\end{aligned}
$$

because by assumption $\boldsymbol{F} \in \mathscr{C}_{*}$. Further, by Proposition 7.1,

$$
\begin{aligned}
\left\|\sum_{m \geq 0} F_{m}\right\|_{\infty} & \leq \sup _{x \in[0,1]} \sum_{m \geq 0} \sum_{(j, k) \in B_{m}}\left|\left\langle F_{m}, \varphi_{j, k}\right\rangle\right|\left|\varphi_{j, k}(x)-\mathbb{E}_{L}\left[\varphi_{j, k}\right]\right| \\
& \leq \sup _{x \in[0,1]} \sum_{j \geq J_{0}} \sup _{m: J_{m}=j} \sup _{(j, k) \in B_{m}}\left|\left\langle F_{m}, \varphi_{j, k}\right\rangle\right| \sum_{k}\left|\varphi_{j, k}(x)-\mathbb{E}_{L}\left[\varphi_{j, k}\right]\right| \\
& \lesssim \sum_{j \geq J_{0}} \sup _{m: J_{m}=j}\left\|F_{m}\right\|_{2} 2^{j / 2} .
\end{aligned}
$$

The conclusion follows by combining equations (7.1) and (7.2).

Proof of Lemma 3.3. We first establish that $\left(\mathscr{S}_{I}\right)_{I \subseteq \mathbb{Z}_{+}}$is a partition of $\mathcal{F}$. Pick $\boldsymbol{F} \in \mathcal{F}$ arbitrary. We want to show that there exists a unique $I \subseteq \mathbb{Z}_{+}$such that $\boldsymbol{F} \in \mathscr{S}_{I}$. We have the following two possibilities:

- If $\left\|F_{0}\right\|_{2} \geq \rho_{0} \Gamma$, choose $I=\{0\} \cup\left\{m \geq 1:\left\|F_{m}\right\|_{2}>\rho_{m} \Gamma \xi^{-1}, J_{m} \leq j_{n}\right\} \cup\{m \geq 1$ : $\left.\left\|F_{m}\right\|_{2}>\gamma 2^{-J_{m}(s+1 / 2)}, J_{m}>j_{n}\right\}$.

- If $\left\|F_{0}\right\|_{2}<\rho_{0} \Gamma$, choose $I=\left\{m \geq 1:\left\|F_{m}\right\|_{2}>\rho_{m} \Gamma, J_{m} \leq j_{n}\right\} \cup\left\{m \geq 1:\left\|F_{m}\right\|_{2}>\right.$ $\left.\gamma 2^{-J_{m}(s+1 / 2)}, J_{m}>j_{n}\right\}$.

The index set $I$ is uniquely defined by $\boldsymbol{F}$, and $\boldsymbol{F} \in \mathscr{S}_{I}$. We now prove the second claim. Let $A:=\bigcup_{I \neq \varnothing} \mathscr{S}_{I}$. We can decompose $A$ as $A_{1} \cup A_{2}$ where $A_{1}:=\bigcup_{I \neq \varnothing, 0 \in I} \mathscr{S}_{I}=\bigcup_{I \subseteq \mathbb{Z}_{+}, 0 \in I} \mathscr{S}_{I}$, and $A_{2}:=\bigcup_{I \neq \varnothing, 0 \notin I} \mathscr{S}_{I}$. Remark that $A_{1}=\left\{\boldsymbol{F} \in \mathcal{F}:\left\|F_{0}\right\|_{2} \geq \rho_{0} \Gamma\right\}$, and $A_{2}=A_{1}^{c} \cap\{\boldsymbol{F} \in \mathcal{F}$ : $\left.\exists m \geq 1,\left\|F_{m}\right\|_{2}>H_{I}(m)\right\}$. Note that if $0 \in I$ then $A_{1}^{c}$ is empty, so

$$
A_{2}=A_{1}^{c} \bigcap\left\{\boldsymbol{F} \in \mathcal{F}: \exists m \geq 1,\left\|F_{m}\right\|_{2} \geq \rho_{m} \Gamma \xi^{-1} \mathbf{1}_{J_{m} \leq j_{n}}+\gamma 2^{-J_{m}(s+1 / 2)} \mathbf{1}_{J_{m}>j_{n}}\right\} .
$$

The conclusion follows since $A^{c}=A_{1}^{c} \cap A_{2}^{c}$ and since $\xi>1$.

Proof of Corollary 3.1: the case where $s>1 / 2$. In view of Lemmas 3.2 and 3.3, it is sufficient to show that

$$
\sum_{j=J_{0}}^{j_{n}} \Gamma \rho_{m} 2^{j / 2}+\sum_{j>j_{n}} \gamma 2^{-j s} \lesssim \tilde{\varepsilon}_{n}^{*}(s) .
$$


But, $\gamma \sum_{j>j_{n}} 2^{-j s} \lesssim \gamma 2^{-j_{n} s} \lesssim(\Gamma / \gamma)^{\frac{2 s}{2 s+1}} \varepsilon_{n}^{*}(s)$ by the definition of $j_{n}$ in equation (3.2). On the other hand $\sum_{j=J_{0}}^{j_{n}} \Gamma \rho_{m} 2^{j / 2} \lesssim \Gamma \sqrt{\log (n) / n} 2^{j_{n} / 2} \lesssim \Gamma(\gamma / \Gamma)^{\frac{1}{2 s+1}} \varepsilon_{n}^{*}(s)$, still by equation (3.2).

Proof of Corollary 3.1: the case where $0<s \leq 1 / 2$. As for the other case, it is enough to show that equation (7.3) holds true. In this cas, $\gamma \sum_{j>j_{n}} 2^{-j s} \lesssim \gamma 2^{-j_{n} s} \lesssim \Gamma \varepsilon_{n}^{*}(s)^{2}$ by equation (3.2). Also, $\sum_{j=J_{0}}^{j_{n}} \Gamma \rho_{m} 2^{j / 2} \leq \Gamma j_{n} \varepsilon_{n}^{*}(s) \lesssim \log \left(\Gamma \varepsilon_{n}^{*}(s) / \gamma\right) \Gamma \varepsilon_{n}^{*}(s) \lesssim \log (n) \varepsilon_{n}^{*}(s)$, again by equation (3.2).

\subsection{Proofs of Propositions 7.1 and 7.2}

Proof of Proposition 7.1, Item (1). By construction we know that there are numbers $a_{j, k} \in \mathbb{R}$ such that $F=\sum_{(j, k) \in B_{m}} a_{j, k}\left(\varphi_{j, k}-\mathbb{E}_{L}\left[\varphi_{j, k}\right]\right)$. We note that if $m \geq 1$ the coefficients $\left(a_{j, k}\right)$ are uniquely determined by $a_{j, k}=\left\langle F, \varphi_{j, k}\right\rangle$, because $J_{m}>J_{0}$ is large enough such that all $\left\langle\varphi_{j, k}, 1\right\rangle=$ 0 for all $(j, k) \in B_{m}$. Thus $\left\langle F, \varphi_{j, k}\right\rangle=\sum_{\left(j^{\prime}, k^{\prime}\right) \in B_{m}} a_{j^{\prime}, k^{\prime}}\left\langle\varphi_{j^{\prime}, k^{\prime}}, \varphi_{j, k}\right\rangle=a_{j, k}$, for any $(j, k) \in$ $B_{m}$. This establishes the proof for $m \geq 1$. For $m=0$, it is the case that $F_{0}$ is in the span of $\left\{\varphi_{j, k}:(j, k) \in B_{0}\right\}$ (because the constants are included in the span), and thus $F_{0}$ can be uniquely written as $F_{0}=\sum_{(j, k) \in B_{0}}\left\langle F_{0}, \varphi_{j, k}\right\rangle \varphi_{j, k}$. But by construction, $\mathbb{E}_{L}\left[F_{0}\right]=0$, so in fact $F_{0}=\sum_{(j, k) \in B_{0}}\left\langle F_{0}, \varphi_{j, k}\right\rangle\left(\varphi_{j, k}-\mathbb{E}_{L}\left[\varphi_{j, k}\right]\right)$.

Proof of Proposition 7.1, Item (2). This follows from the Item (1) and because for $(j, k) \notin B_{0}$ and $J_{0}$ large enough, we have $\left\langle 1, \varphi_{j, k}\right\rangle=0$. Therefore, it it the case that $\left\langle F, \varphi_{j, k}\right\rangle=\sum_{\left(j^{\prime}, k^{\prime}\right) \in B_{m}}\left\langle F, \varphi_{j^{\prime}, k^{\prime}}\right\rangle\left\langle\varphi_{j^{\prime}, k^{\prime}}, \varphi_{j, k}\right\rangle$. By orthogonality of the wavelet basis, the previous is either 0 if $(j, k) \notin B_{m}$, or $\left\langle F, \varphi_{j, k}\right\rangle$ otherwise.

Proof of Proposition 7.1, Item (3). The lower bound is immediate because $\|\left. F\right|_{2} ^{2}=\sum_{(j, k) \in \mathcal{V}}\left\langle F, \varphi_{j, k}\right\rangle^{2} \geq$ $\sum_{(j, k) \in B_{m}}\left\langle F, \varphi_{j, k}\right\rangle^{2}$, so indeed $C_{1}=1$ works. For the upper bound, we note that because $\|L\|_{\infty} \lesssim 1$ we have $\left\|F_{m}\right\|_{2}^{2} \asymp \mathbb{E}_{L}\left[F_{m}^{2}\right]$, and by Item (1)

$$
\begin{aligned}
\mathbb{E}_{L}\left[F_{m}^{2}\right] & =\mathbb{E}_{L}\left[\left(\sum_{(j, k) \in B_{m}}\left\langle F, \varphi_{j, k}\right\rangle \varphi_{j, k}-\mathbb{E}_{L}\left[\sum_{(j, k) \in B_{m}}\left\langle F, \varphi_{j, k}\right\rangle \varphi_{j, k}\right]\right)^{2}\right] \\
& \leq \mathbb{E}_{L}\left[\left(\sum_{(j, k) \in B_{m}}\left\langle F, \varphi_{j, k}\right\rangle \varphi_{j, k}\right)^{2}\right] \\
& \lesssim\left\|\sum_{(j, k) \in B_{m}}\left\langle F, \varphi_{j, k}\right\rangle \varphi_{j, k}\right\|_{2}^{2} \\
& =\sum_{(j, k) \in B_{m}}\left\langle F, \varphi_{j, k}\right\rangle^{2},
\end{aligned}
$$

where the last line follows by the orthogonality of the wavelet basis.

Proof of Proposition 7.1, Item (4). We start with the upper bound, which follows from similar arguments than those of the Item (3). Indeed, recall that $\mathbb{E}_{L}\left[g^{2}\right] \asymp\|g\|_{2}^{2}$ for all $g$ because $\|L\|_{\infty} \lesssim 1$, hence

$$
\mathbb{E}_{L}\left[\left(\sum_{m \in J} F_{m}\right)^{2}\right]=\mathbb{E}_{L}\left[\left(\sum_{m \in J} \sum_{(j, k) \in B_{m}}\left\langle F_{m}, \varphi_{j, k}\right\rangle \varphi_{j, k}-\mathbb{E}_{L}\left[\sum_{m \in J} \sum_{(j, k) \in B_{m}}\left\langle F_{m}, \varphi_{j, k}\right\rangle \varphi_{j, k}\right]\right)^{2}\right]
$$




$$
\begin{aligned}
& \leq \mathbb{E}_{L}\left[\left(\sum_{m \in J} \sum_{(j, k) \in B_{m}}\left\langle F_{m}, \varphi_{j, k}\right\rangle \varphi_{j, k}\right)^{2}\right] \\
& \lesssim\left\|\sum_{m \in J} \sum_{(j, k) \in B_{m}}\left\langle F_{m}, \varphi_{j, k}\right\rangle \varphi_{j, k}\right\|_{2}^{2} \\
& =\sum_{m \in J} \sum_{(j, k) \in B_{m}}\left\langle F_{m}, \varphi_{j, k}\right\rangle^{2}
\end{aligned}
$$

Then, by the Item (2),

$$
\mathbb{E}_{L}\left[\left(\sum_{m \in J} F_{m}\right)^{2}\right] \lesssim \sum_{m \in J}\left\|F_{m}\right\|_{2}^{2} .
$$

We now proceed with the lower bound. By the Item (1),

$$
\begin{aligned}
\left\|\sum_{m \in J} F_{m}\right\|_{2}^{2} & =\sum_{(j, k) \in \mathcal{V}}\left\langle\sum_{m^{\prime} \in J} F_{m^{\prime}}, \varphi_{j, k}\right\rangle^{2} \\
& \geq \sum_{m \geq 1} \sum_{(j, k) \in B_{m}}\left\langle\sum_{m^{\prime} \in J} \sum_{\left(j^{\prime}, k^{\prime}\right) \in B_{m^{\prime}}}\left\langle F_{m^{\prime}}, \varphi_{j^{\prime}, k^{\prime}}\right\rangle\left(\varphi_{j^{\prime}, k^{\prime}}-\mathbb{E}_{L}\left[\varphi_{j^{\prime}, k^{\prime}}\right]\right), \varphi_{j, k}\right\rangle^{2} \\
& =\sum_{m \geq 1} \sum_{(j, k) \in B_{m}}\left\langle\sum_{m^{\prime} \in J} \sum_{\left(j^{\prime}, k^{\prime}\right) \in B_{m^{\prime}}}\left\langle F_{m^{\prime}}, \varphi_{j^{\prime}, k^{\prime}}\right\rangle \varphi_{j^{\prime}, k^{\prime}}, \varphi_{j, k}\right\rangle^{2} \\
& =\sum_{m \in J} \sum_{(j, k) \in B_{m}}\left\langle F_{m}, \varphi_{j, k}\right\rangle^{2} \mathbf{1}_{m \neq 0},
\end{aligned}
$$

where the third line follows because $\left\langle 1, \varphi_{j, k}\right\rangle=0$ for all $(j, k) \notin B_{0}$, and the last line by orthogonality of the wavelet basis. Therefore by the Item (3) it must be the case that

$$
\mathbb{E}_{L}\left[\left(\sum_{m \in J} F_{m}\right)^{2}\right] \gtrsim \sum_{m \in J}\left\|F_{m}\right\|_{2}^{2} \mathbf{1}_{m \neq 0} .
$$

The last display gives the proof in the case where $0 \notin J$. We now assume that $0 \in J$, which is a more delicate case. In this situation, we have that $F_{0}=\sum_{m \in J} F_{m}-\sum_{m \in J} F_{m} \mathbf{1}_{m \neq 0}$, and thus

$$
\begin{aligned}
\mathbb{E}_{L}\left[F_{0}^{2}\right] & \leq 2 \mathbb{E}_{L}\left[\left(\sum_{m \in J} F_{m}\right)^{2}\right]+2 \mathbb{E}_{L}\left[\left(\sum_{m \in J} F_{m} \mathbf{1}_{m \neq 0}\right)^{2}\right] \\
& \lesssim \mathbb{E}_{L}\left[\left(\sum_{m \in J} F_{m}\right)^{2}\right]+\sum_{m \in J}\left\|F_{m}\right\|_{2}^{2} \mathbf{1}_{m \neq 0},
\end{aligned}
$$

where the second line follows from the upper bound of equation (7.4) applied to the index set $J \backslash\{0\}$. Combining equations (7.5) and (7.6),

$$
\left\|F_{0}\right\|_{2}^{2} \lesssim \mathbb{E}_{L}\left[F_{0}^{2}\right] \lesssim \mathbb{E}_{L}\left[\left(\sum_{m \in J} F_{m}\right)^{2}\right] .
$$


Now if we combine the equations (7.5) and (7.7), we have indeed

$$
\begin{aligned}
\mathbb{E}_{L}\left[\left(\sum_{m \in J} F_{m}\right)^{2}\right] & \gtrsim \max \left\{\sum_{m \in J}\left\|F_{m}\right\|_{2}^{2} \mathbf{1}_{m \neq 0},\left\|F_{0}\right\|_{2}^{2}\right\} \\
& \geq \frac{1}{2} \sum_{m \in J}\left\|F_{m}\right\|_{2}^{2} \mathbf{1}_{m \neq 0}+\frac{1}{2}\left\|F_{0}\right\|_{2}^{2} \\
& =\frac{1}{2} \sum_{m \in J}\left\|F_{m}\right\|_{2}^{2} .
\end{aligned}
$$

Proof of Proposition 7.1, Item (5). The first claim is a well-known localization properties of the wavelet basis. The second fact follows because $\mathbb{E}_{L}\left[\varphi_{j, k}\right] \leq\left\|p_{L}\right\|_{\infty}\left\|\varphi_{j, k}\right\|_{1} \lesssim\left\|p_{L}\right\|_{\infty} 2^{-j / 2}$, and because there are no more than $2^{j}$ wavelets at each level $j \geq 0$. The third fact is obvious.

Proof of Proposition 7.2. From the definition of $\mathcal{E}_{I}$ and from Proposition 7.1-(4), it is immediate that $\mathcal{E}_{I}^{2} \gtrsim \sum_{m \in I} H_{I}(m)^{2}$. If $s>1 / 2$ then the result is immediate. In case $0<s \leq 1 / 2$, then we note that by definition of $j_{n}$

$$
\gamma 2^{-j_{n}(s+1 / 2)} \geq \Gamma 2^{-j_{n} / 2} \varepsilon_{n}^{*}(s) \Longrightarrow 2^{-j_{n}} \geq\left(\frac{\Gamma}{\gamma}\right)^{\frac{1}{s}}\left(\frac{\log (n)}{n}\right)^{\frac{1}{2 s+1}}
$$

Therefore,

$$
\begin{aligned}
\sum_{m \in I} \rho_{m}^{2} \mathbf{1}_{J_{m} \leq j_{n}} & =\sum_{m \in I} 2^{-J_{m}} \varepsilon_{n}^{*}(s)^{2} \mathbf{1}_{J_{m} \leq j_{n}} \\
& \geq 2^{-j_{n}} \varepsilon_{n}^{*}(s)^{2} \sum_{m \in I} \mathbf{1}_{J_{m} \leq j_{n}} \\
& =\left(\frac{\Gamma}{\gamma}\right)^{\frac{1}{s}} \cdot \frac{\log (n)}{n} \sum_{m \in I} \mathbf{1}_{J_{m} \leq j_{n}} \\
& \geq \frac{\log (n)}{n} \sum_{m \in I} \mathbf{1}_{J_{m} \leq j_{n}},
\end{aligned}
$$

where the last line is true under Assumption 1.

\section{Acknowledgements}

This work was supported by U.S. Air Force Office of Scientific Research grant \#FA9550-15-1-0074. The author also thanks Daniel M. Roy for helpful discussions and the opportunity to work on this project.

\section{References}

Barron, A., Schervish, M. J. and WASSERman, L. (1999). The consistency of posterior distributions in nonparametric problems. Ann. Statist. 27 536-561. 
Boucheron, S., Lugosi, G. and MAssart, P. (2013). Concentration inequalities: A nonasymptotic theory of independence. Oxford university press.

BROWN, L. D., ZHANG, C.-H. et al. (1998). Asymptotic nonequivalence of nonparametric experiments when the smoothness index is 1/2. The Annals of Statistics 26 279-287.

CAstillo, I. (2014). On Bayesian supremum norm contraction rates. Ann. Statist. 42 2058-2091.

Castillo, I. (2017). Pólya tree posterior distributions on densities. In Annales de l'Institut Henri Poincaré, Probabilités et Statistiques 53 2074-2102. Institut Henri Poincaré.

Castillo, I. and Mismer, R. (2019). Spike and Slab Pólya tree posterior distributions. arXiv preprint arXiv:1911.12106.

Cohen, A., Daubechies, I. and Vial, P. (1993). Wavelet bases on the interval and fast algorithms. Journal of Applied and Computational Harmonic Analysis 1 54-81.

Donoho, D. L., Johnstone, I. M., Kerkyacharian, G. and PiCARD, D. (1996). Density estimation by wavelet thresholding. Ann. Statist. 508-539.

Ghosal, S., Ghosh, J. K. and VAn Der VAART, A. W. (2000). Convergence rates of posterior distributions. Ann. Statist. 28 500-531.

GHOSAL, S. and VAN DER VAART, A. (2007a). Posterior convergence rates of Dirichlet mixtures at smooth densities. Ann. Statist. 35 697-723.

GHOSAL, S. and VAN DER VAART, A. (2007b). Convergence rates of posterior distributions for noniid observations. Ann. Statist. 35 192-223.

GINÉ, E. and NICKL, R. (2011). Rates of contraction for posterior distributions in $L^{r}$-metrics, $1 \leq$ $r \leq \infty$. Ann. Statist. 39 2883-2911.

GINÉ, E. and NICKL, R. (2016). Mathematical foundations of infinite-dimensional statistical models 40. Cambridge University Press.

Goldenshluger, A. and LePski, O. (2014). On adaptive minimax density estimation on $\mathbb{R}^{d}$. Probability Theory and Related Fields 159 479-543.

Härdle, W., Kerkyacharian, G., PicArd, D. and TSybAKov, A. (2000). Wavelets, approximation, and statistical applications. Springer Science \& Business Media.

HASMINSKII, R. Z. (1979). A lower bound on the risks of non-parametric estimates of densities in the uniform metric. Theory of Probability \& Its Applications 23 794-798.

Hoffmann, M., Rousseau, J. and SchmidT-Hieber, J. (2015). On adaptive posterior concentration rates. Ann. Statist. 43 2259-2295.

IBRAgimov, I. A. and HASMINSKII, R. Z. (1980). On estimate of the density function. Zapiski Nauchnykh Seminarov POMI 98 61-85.

Lijoi, A., Prünster, I. and Walker, S. G. (2005). On consistency of nomparametric normal mixtures for Bayesian density estimation. Journal of the American Statistical Association $1001292-$ 1296.

Mitchell, T. J. and BeAuchamp, J. J. (1988). Bayesian variable selection in linear regression. Journal of the American Statistical Association 83 1023-1032.

POLLARD, D. (1990). Empirical processes: theory and applications. In NSF-CBMS regional conference series in probability and statistics $\mathrm{i}-86$. JSTOR.

Rivoirard, V. and Rousseau, J. (2012). Bernstein-von Mises theorem for linear functionals of the density. Ann. Statist. 40 1489-1523.

SCHWARTZ, L. (1965). On Bayes procedures. Zeitschrift für Wahrscheinlichkeitstheorie und verwandte Gebiete 4 10-26.

SCRICCIOLO, C. (2014). Adaptive Bayesian Density Estimation in $L^{p}$-metrics with Pitman-Yor or Normalized inverse-Gaussian Process Kernel Mixtures. Bayesian Analysis 9 475-520.

Shen, X., WASSERman, L. et al. (2001). Rates of convergence of posterior distributions. Ann. Statist. 29 687-714. 
WALKer, S. G., LIJOI, A. and PRÜNSTER, I. (2007). On rates of convergence for posterior distributions in infinite-dimensional models. Ann. Statist. 738-746.

Yoo, W. W. and Ghosal, S. (2016). Supremum norm posterior contraction and credible sets for nonparametric multivariate regression. Ann. Statist. 44 1069-1102.

Yoo, W. W., Rousseau, J. and Rivoirard, V. (2017). Adaptive Supremum Norm Posterior Contraction: Spike-and-Slab Priors and Anisotropic Besov Spaces. arXiv preprint arXiv:1708.01909. 


\title{
Adaptive Bayesian density estimation in sup-norm : Supplementary Material
}

\author{
ZACHARIE NAULET
}

Université Paris-Saclay

Laboratoire de mathématiques d'Orsay

91405, Orsay, France

E-mail: zacharie.nauleteuniversite-paris-saclay.fr

\section{S1. Organization}

This document is supplementary material for the article Adaptive Bayesian density estimation in supnorm. It contains the missing proofs for the spike-and-slab prior example. We refer to the main document for all the definitions.

- In Section S2, we introduce some new notations that were not needed in the main document, but which we will need in the supplemental.

- In Section S3, we prove the posterior concentration on small Hellinger neighborhoods of the true density. This is the first step toward concentration in stronger distances.

- In Section S4, we prove posterior concentration on $\mathbb{L}_{2}$ neighborhoods and uniform posterior consistency, which is needed to prove the Lemma 3.1 stated in the main document.

- In Section S5.1, we give the proofs that are missing in the main document. In particular, the Lemma 3.1 establishing the posterior concentration on $\mathscr{C}_{*}$ is proved in Section S5.1, the Lemma 6.4 controlling the covariance terms is proved in Section S5.2, and finally Lemmas 5.1 and 5.2 are proved in Section S5.3.

Every section, subsection, theorem, etc. of the supplemental has label prefixed by $\mathrm{S}$ and is cited in cyan. References to the main document are cited in blue with no prefix.

\section{S2. Notations}

We use the same conventions as in the main paper. We furthermore make use of the following measures of discrepancy between probability distributions. The Kullback-Leibler divergence is written $\mathrm{KL}(P, Q):=\int_{0}^{1} p \log (p / q)$. We also use the second-order measure of discrepancy $\mathrm{V}(P, Q):=$ $\int_{0}^{1} p \log ^{2}(p / q)$.

For convenience, we also define the following sequence norms on $\Theta$. We denote the usual $\ell_{2}$ norm by $\|\boldsymbol{\theta}\|_{2}^{2}:=\sum_{(j, k) \in \mathcal{V}}\left|\theta_{j, k}\right|^{2}$. In addition, we define the mixed $\ell_{1, \infty}$ norm such that $\|\boldsymbol{\theta}\|_{1, \infty}:=$ $\sum_{j \geq 0} \max _{k}\left|\theta_{j, k}\right| 2^{j / 2}$. It is a well-known fact that if $f=\sum_{(j, k) \in \mathcal{V}} \theta_{j, k} \varphi_{j, k}$, then $\|f\|_{\infty} \lesssim\|\boldsymbol{\theta}\|_{1, \infty}$, and $\|f\|_{2}=\|\boldsymbol{\theta}\|_{2}$. 


\section{S3. Posterior concentration on Hellinger balls}

The starting point to the proof of the concentration of the posterior in strong distances is to first establish the contraction of the posterior for $P_{\boldsymbol{\theta}}$ on small Hellinger neighborhoods of $P_{L}$. We will prove that for $\varepsilon_{n}^{*}(s):=(\log (n) / n)^{s /(2 s+1)}$, the spike-and-slab log-density prior satisfies for $M>0$ large enough,

$$
\sup _{L \in \Sigma(R, s)} \mathbb{E}_{L} \Pi\left(\boldsymbol{\theta}: \mathrm{H}\left(P_{\boldsymbol{\theta}}, P_{L}\right)>M \varepsilon_{n}^{*}(s) \mid \mathbf{X}_{n}\right)=o(1), \quad n \rightarrow \infty .
$$

This will prove the Theorem 2.1. One can notice that $\varepsilon_{n}^{*}(s)$ is not the optimal rate for the Hellinger distance. This is a well-known consequence of the fact that prior independence of the wavelets coefficients cannot yield optimal contraction on Hellinger or $\mathbb{L}_{2}$ balls (Hoffmann, Rousseau and Schmidt-Hieber, 2015; Cai, 2008).

Equation (S3.1) is obtained classically, as a consequence of Ghosal, Ghosh and van der Vaart (2000, Theorem 2.1) combined with Propositions S3.1 and S3.2 below.

Proposition S3.1. Let $\Pi$ be the spike-and-slab prior described in Section 2.2. Assume $L \in \Sigma(R, s)$ for some $0<s_{0} \leq s \leq S$ and let $\varepsilon_{n}^{*}(s):=(\log (n) / n)^{s /(2 s+1)}$. Then, there exists $C>0$ such that for $n$ large enough,

$$
\Pi\left(\mathrm{KL}\left(P_{L}, P_{\boldsymbol{\theta}}\right) \leq \varepsilon_{n}^{*}(s)^{2}, \mathrm{~V}\left(P_{L}, P_{\boldsymbol{\theta}}\right) \leq \varepsilon_{n}^{*}(s)^{2}\right) \geq \exp \left\{-C n \varepsilon_{n}^{*}(s)^{2}\right\} .
$$

Proof. Let $\boldsymbol{\theta}$ be such that $\left|\theta_{j, k}-\theta_{j, k}^{L}\right| \leq n^{-1 / 2}$ if $j \leq \tilde{J}$ and $\theta_{j, k}=0$ for $g>\tilde{J}$, with $\tilde{J}$ a truncation level to be chosen accordingly. Because $\left|\theta_{j, k}^{L}\right| \leq R 2^{-j(s+1 / 2)}$ by assumption, this implies that $\| \boldsymbol{\theta}-$ $\boldsymbol{\theta}^{L} \|_{1, \infty} \lesssim \sum_{j \leq \tilde{J}} 2^{j / 2} n^{-1 / 2}+\sum_{j>\tilde{J}^{2}} 2^{-j s} \lesssim 2^{\tilde{J} / 2} n^{-1 / 2}+2^{-\tilde{J} s} \lesssim \varepsilon_{n}^{*}(s)$ by choosing the optimal truncation level. By van der Vaart and van Zanten (2008, Lemma 3.1), this implies that $\mathrm{KL}\left(P_{L}, P_{\boldsymbol{\theta}}\right) \lesssim$ $\varepsilon_{n}^{*}(s)^{2}$ and $\mathrm{V}\left(P_{L}, P_{\boldsymbol{\theta}}\right) \lesssim \varepsilon_{n}^{*}(s)^{2}$. Thus, for some $B>0$,

$$
\begin{aligned}
\Pi\left(\mathrm{KL}\left(P_{L}, P_{\boldsymbol{\theta}}\right) \leq B \varepsilon_{n}^{*}(s)^{2},\right. & \left.\mathrm{V}\left(P_{L}, \boldsymbol{\theta}\right) \leq B \varepsilon_{n}^{*}(s)^{2}\right) \\
& \geq \prod_{j \leq \tilde{J}} \prod_{k} \omega_{j} \Pi_{j, k}\left(\left|\theta_{j, k}-\theta_{j, k}^{L}\right| \leq n^{-1 / 2}\right) \prod_{j=\tilde{J}}^{J_{n}} \prod_{k}\left(1-\omega_{j}\right) \\
& \geq \prod_{j \leq \tilde{J}} \prod_{k} \omega_{j} 2^{j\left(s_{0}+1 / 2\right)} \int_{\theta_{j, k}^{L}-n^{-1 / 2}}^{\theta_{j, k}^{L}+n^{-1 / 2}} f\left(2^{j\left(s_{0}+1 / 2\right)} t\right) \mathrm{d} t \prod_{j=\tilde{J}}^{J_{n}} \prod_{k}\left(1-\omega_{j}\right) \\
& =\prod_{j \leq \tilde{J}} \prod_{k} \omega_{j} \int_{2^{j\left(s_{0}+1 / 2\right)}\left(\theta_{j, k}^{L}-n^{-1 / 2}\right)}^{2^{j\left(s_{0}+1 / 2\right)}} f(t) \mathrm{d} t \prod_{j, k}^{L}+\prod_{j=\tilde{J}}\left(1-\omega_{j}\right) .
\end{aligned}
$$

Since $s \geq s_{0}$ and $j \leq \tilde{J}$, there is a constant $g>0$ such that $f \geq g$ always on the domain of integration of the previous display. Moreover, $\omega_{j} \gtrsim e^{-j b_{1}}$ and there are no more than a generic constant times $2^{j}$ wavelets at each level $j$, then for some constants $C>0$,

$$
\Pi\left(\mathrm{KL}\left(P_{L}, P_{\boldsymbol{\theta}}\right) \leq B \varepsilon_{n}^{*}(s)^{2}, \mathrm{~V}\left(P_{L}, P_{\boldsymbol{\theta}}\right) \leq B \varepsilon_{n}^{*}(s)^{2}\right) \geq \exp \left\{-C 2^{\tilde{J}} \log (n)\right\}
$$

The conclusion follows since $2^{\tilde{J}} \log (n) \lesssim n \varepsilon_{n}^{*}(s)^{2}$. 
Proposition S3.2. Let $\Pi$ be the spike-and-slab prior described in Section 2.2. Let $n \varepsilon_{n}^{2} \rightarrow \infty$ with $\log \left(n \varepsilon_{n}^{2}\right) \gtrsim \log (n)$ and let $n$ be large enough. Then for every $C>0$ there exists a sequence of sets $\left(\Theta_{n}\right)_{n \geq 0}$ such that $\Pi\left(\Theta_{n}\right) \lesssim \exp \left\{-C n \varepsilon_{n}^{2}\right)$ and $\log N\left(\varepsilon_{n}, \mathcal{P}_{n}, \mathrm{H}\right) \leq n \varepsilon_{n}^{2}$, where $\mathcal{P}_{n}=\left\{p_{\boldsymbol{\theta}}\right.$ : $\left.\boldsymbol{\theta} \in \Theta_{n}\right\}$.

Proof. We choose, for some constant $K, K^{\prime}>0$,

$$
\Theta_{n}:=\left\{\boldsymbol{\theta} \in \Theta: \begin{array}{c}
\sup _{j, k} 2^{j\left(s_{0}+1 / 2\right)}\left|\theta_{j, k}\right| \leq \log \left(K n \varepsilon_{n}^{2}\right) \\
\left|\left\{\theta_{j, k} \neq 0\right\}\right| \leq K^{\prime} n \varepsilon_{n}^{2} / \log \left(n \varepsilon_{n}^{2}\right)
\end{array}\right\} .
$$

Then, by construction of the prior since $\theta_{j, k}=2^{-j\left(s_{0}+1 / 2\right)} Z_{j, k}$ for $Z_{j, k} \sim F$, and since all the coefficients are independent,

$$
\begin{aligned}
\Pi\left(\Theta_{n}^{c}\right) & \leq \sum_{j, k} \omega_{j} \Pi\left(\left|Z_{j, k}\right|>\log \left(K n \varepsilon_{n}^{2}\right)\right)+\Pi\left(\left|\left\{\theta_{j, k} \neq 0\right\}\right|>\frac{K^{\prime} n \varepsilon_{n}^{2}}{\log \left(n \varepsilon_{n}^{2}\right)}\right) \\
& \leq e^{-b_{2} K n \varepsilon_{n}^{2}}+\Pi\left(\left|\left\{\theta_{j, k} \neq 0\right\}\right|>\frac{K^{\prime} n \varepsilon_{n}^{2}}{\log \left(n \varepsilon_{n}^{2}\right)}\right),
\end{aligned}
$$

where the second line follows because $\omega_{j} \leq 2^{-j\left(1+\mu_{\star}\right)}$ for $\mu_{\star}>0$ and because there are at most a generic constant times $2^{j}$ wavelets at each level $j \in \mathbb{Z}_{+}$, and because of the assumption of equation (2.3). Furthermore, $\mathbb{E}_{\Pi}\left[\left|\left\{\theta_{j, k} \neq 0\right\}\right|\right] \leq \sum_{j=0}^{J_{n}} \sum_{k} 2^{-j\left(1+\mu_{\star}\right)} \lesssim 1$, Hence, by Chernoff's bound, for some constant $B>0$ when $K^{\prime} n \varepsilon_{n}^{2}$ gets large enough

$$
\Pi\left(\left|\left\{\theta_{j, k} \neq 0\right\}\right|>\frac{K^{\prime} n \varepsilon_{n}^{2}}{\log \left(n \varepsilon_{n}^{2}\right)}\right) \leq e^{-B K^{\prime} n \varepsilon_{n}^{2}} .
$$

Thus $\Theta_{n}$ meets the first requirement of the proposition.

We now determine an upper bound on $N\left(\varepsilon_{n}, \mathcal{P}_{n}, \mathrm{H}\right)$. We assume without loss of generality that $M_{n}:=K^{\prime} n \varepsilon_{n}^{2} / \log \left(n \varepsilon_{n}^{2}\right)$ is integer. Furthermore there is a generic constant $c>0$ such that, for any $\boldsymbol{\theta}, \boldsymbol{\theta}^{\prime} \in \Theta_{n}$ (see van der Vaart and van Zanten, 2008, Lemma 3.1),

$$
\mathrm{H}\left(P_{\boldsymbol{\theta}}, P_{\boldsymbol{\theta}^{\prime}}\right) \lesssim\left\|\boldsymbol{\theta}-\boldsymbol{\theta}^{\prime}\right\|_{1, \infty} e^{-c\left\|\boldsymbol{\theta}-\boldsymbol{\theta}^{\prime}\right\|_{1, \infty}}
$$

But, for any $\boldsymbol{\theta}, \boldsymbol{\theta}^{\prime} \in \Theta_{n}$, writing $Z_{j, k}=2^{j\left(s_{0}+1 / 2\right)} \theta_{j, k}$ and $Z_{j, k}^{\prime}=2^{j\left(s_{0}+1 / 2\right)} \theta_{j, k}^{\prime}$, it is clear that $\| \boldsymbol{\theta}-$ $\boldsymbol{\theta}^{\prime}\left\|_{1, \infty} \lesssim\right\| \boldsymbol{Z}-\boldsymbol{Z}^{\prime} \|_{\infty}$. Since $\boldsymbol{\theta}$ has no more than $M_{n}$ non-zero entries, $N\left(\varepsilon_{n}, \mathcal{P}_{n}, \boldsymbol{H}\right)$ is no more than the sum over all possible subsets of indices $I \subseteq A_{n}:=\left\{(j, k): j \leq \frac{\log (n)}{\log (2)}\right\}$ such that $|I| \leq M_{n}$ of the covering numbers of

$$
E_{I}:=\left\{\boldsymbol{z} \in \mathbb{R}^{I}:\|\boldsymbol{z}\|_{\infty} \leq \log \left(K n \varepsilon_{n}^{2}\right)\right\}
$$

with balls of radius $B \varepsilon_{n}$, for a universal $B>0$. A $B \varepsilon_{n}$-net over $E_{I}$ has cardinality no more than

$$
\left(\frac{\log \left(K n \varepsilon_{n}^{2}\right)}{B \varepsilon_{n}}\right)^{|I|} \leq\left(\frac{\log \left(K n \varepsilon_{n}^{2}\right)}{B \varepsilon_{n}}\right)^{M_{n}} \lesssim e^{C_{1} K^{\prime} n \varepsilon_{n}^{2}}
$$


for some universal $C_{1}>0$. The number of possible subsets is $\sum_{m=0}^{M_{n}}\left(\begin{array}{c}\left|A_{n}\right| \\ m\end{array}\right)$. Using the well-known inequality $\left(\begin{array}{l}n \\ k\end{array}\right) \leq \frac{n^{k}}{k !}$, we deduce that,

$$
\sum_{m=0}^{M_{n}}\left(\begin{array}{c}
\left|A_{n}\right| \\
m
\end{array}\right) \leq \sum_{m=0}^{M_{n}} \frac{\left|A_{n}\right|^{m}}{m !}=\frac{e^{\left|A_{n}\right|}}{M_{n} !} \int_{\left|A_{n}\right|}^{\infty} t^{M_{n}} e^{-t} \mathrm{~d} t .
$$

By Stirling's formula, as $n \rightarrow \infty$, because $\left|A_{n}\right| \gg M_{n}$,

$$
\begin{aligned}
\sum_{m=0}^{M_{n}}\left(\begin{array}{c}
\left|A_{n}\right| \\
m
\end{array}\right) & \leq \frac{(1+o(1)) e^{\left|A_{n}\right|+M_{n}-M_{n} \log M_{n}}}{\sqrt{2 \pi M_{n}}} \int_{\left|A_{n}\right|}^{\infty} t^{M_{n}} e^{-t} \mathrm{~d} t \\
& =\frac{(1+o(1))\left|A_{n}\right| e^{\left|A_{n}\right|+M_{n}-M_{n} \log M_{n}+M_{n} \log \left|A_{n}\right|}}{\sqrt{2 \pi M_{n}}} \int_{1}^{\infty} e^{-u\left|A_{n}\right|+M_{n} \log (u)} \mathrm{d} u \\
& \leq \frac{(1+o(1))\left|A_{n}\right| e^{\left|A_{n}\right|+M_{n}-M_{n} \log M_{n}+M_{n} \log \left|A_{n}\right|}}{\sqrt{2 \pi M_{n}}} \int_{1}^{\infty} e^{-u\left|A_{n}\right|\left(1-\frac{M_{n}}{e\left|A_{n}\right|}\right)} \mathrm{d} u \\
& =\frac{(1+o(1)) e^{M_{n}\left(1+e^{-1}\right)-M_{n} \log M_{n}+M_{n} \log \left|A_{n}\right|}}{\sqrt{2 \pi M_{n}}\left(1-\frac{M_{n}}{e\left|A_{n}\right|}\right)}
\end{aligned}
$$

Since $M_{n} \asymp \frac{K^{\prime} n \varepsilon_{n}^{2}}{\log \left(n \varepsilon_{n}^{2}\right)}$ and $\left|A_{n}\right| \lesssim n$, it follows for some universal constant $C_{2}>0$ that $\sum_{m=0}^{M_{n}}\left(\begin{array}{c}\left|A_{n}\right| \\ m\end{array}\right) \lesssim$ $e^{C_{2} K^{\prime} n \varepsilon_{n}^{2}}$. The conclusion of the proposition follows by picking $K^{\prime}$ small enough.

\section{S4. Posterior concentration on $L^{2}$ balls and uniform consistency}

Here we strengthen a little bit the result of Section S3 and we show that the posterior indeed concentrates on $\|\cdot\|_{2}$ and $\|\cdot\|_{1, \infty}$ neighborhoods of $\boldsymbol{\theta}^{L}$. Indeed, since the coefficients $\boldsymbol{\theta}^{L}$ is only identifiable up to suitable translation, we don't expect to concentrates on balls of the form $\left\{\boldsymbol{\theta}:\left\|\boldsymbol{\theta}-\boldsymbol{\theta}^{L}\right\| \leq R\right\}$, but instead on balls of the form $\left\{\boldsymbol{\theta}:\left\|\boldsymbol{\theta}+\boldsymbol{\Xi}^{\boldsymbol{\theta}}-\boldsymbol{\theta}^{L}\right\| \leq R\right\}$, where $\boldsymbol{\Xi}^{\boldsymbol{\theta}}$ is the vector with entries $\Xi_{j, k}^{\boldsymbol{\theta}}:=\left\langle\xi^{\boldsymbol{\theta}}, \varphi_{j, k}\right\rangle$, where $\xi^{\boldsymbol{\theta}}$ is the log-normalizing constant defined in Section 2.1. We remark that by the properties of the wavelet basis, there is a $J_{0}$ such that $\Xi_{j, k}=0$ for all $j \geq J_{0}$ and all $k$. We also prove that concentration on $\|\cdot\|_{1, \infty}$ balls imply the posterior concentration on $\mathscr{C}_{*}$ as required in the main paper.

The proof follows a minor adaptation of Castillo (2014, Lemma 4), itself inspired from ideas of Rivoirard and Rousseau (2012). The argument, however, requires to be adapted to handle the fact that there is a non zero prior mass of having coefficients $\theta_{j, k} \neq 0$ with $j>\tilde{J}$, with $\tilde{J}$ being the optimal truncation level.

Proposition S4.1. Let $\Pi$ be the spike-and-slab prior described in Section 2.2. Then, there is a generic constant $C>0$ such that,

$$
\sup _{J \geq 0} \mathbb{E}_{L} \Pi\left(\sum_{j>J} \max _{k}\left|\theta_{j, k}\right| 2^{j / 2}>C 2^{-J s_{0}} \log (n) \mid \mathbf{X}_{n}\right)=o(1) .
$$


Proof. By construction, $\theta_{j, k}=2^{-j\left(s_{0}+1 / 2\right)} Z_{j, k}$, where $Z_{j, k} \stackrel{i i d}{\sim} F$. Then, we see that $\sum_{j>J} \max _{k}\left|\theta_{j, k}\right| 2^{j / 2}=$ $\sum_{j>J} 2^{-j s_{0}} \max _{k}\left|Z_{j, k}\right| \lesssim 2^{-J s_{0}} \sup _{v}\left|Z_{v}\right|$. Hence, the probability in the statement of the proposition is bounded by $\mathbb{E}_{L} \Pi\left(\sup _{v}\left|Z_{v}\right| \gtrsim \log (n) \mid \mathbf{X}_{n}\right)$. But, by the assumptions on $F$, we have that for any $K>0$

$$
\begin{aligned}
\Pi\left(\sup _{v}\left|Z_{v}\right|>\log \left(K n \varepsilon_{n}^{*}(s)^{2}\right)\right) & \leq \sum_{j \geq 0} \sum_{k} \Pi\left(\left|Z_{j, k}\right|>\log \left(K n \varepsilon_{n}^{*}(s)^{2}\right)\right) \\
& =\sum_{j \geq 0} \sum_{k} \omega_{j} \Pi\left(\left|Z_{j, k}\right|>\log \left(K n \varepsilon_{n}^{*}(s)^{2}\right) \mid \theta_{j, k} \neq 0\right) \\
& \lesssim \exp \left\{-b_{2} K n \varepsilon_{n}^{*}(s)^{2}\right\}
\end{aligned}
$$

where the last line follows because $\omega_{j} \leq 2^{-j\left(1+\mu_{\star}\right)}$ for $\mu_{\star}>0$ by assumption, and there are at most a generic constant times $2^{j}$ wavelets at each level $j$. By Ghosal, Ghosh and van der Vaart (2000, see the proof of Theorem 2.1) the last display together with Proposition S3.1 implies that the proposition is true, because $\log \left(n \varepsilon_{n}^{*}(s)^{2}\right) \asymp \log (n)$ by definition of $\varepsilon_{n}^{*}(s)$.

We are now in position to adapt Castillo (2014, Lemma 4) to our setting. The idea is to leverage the property that the high frequency coefficients are always small enough (Proposition S4.1) to obtain that the Hellinger contraction implies the desired result.

Proposition S4.2. Let $\Pi$ be the spike-and-slab prior described in Section 2.2 and assume $0<s_{0} \leq$ $s \leq S$. Then, under the assumption of the paper, for all $R>0$ the following holds,

$$
\begin{gathered}
\exists K>0, \quad \sup _{L \in \Sigma(R, s)} \mathbb{E}_{L} \Pi\left(\left\|\boldsymbol{\theta}+\boldsymbol{\Xi}^{\boldsymbol{\theta}}-\boldsymbol{\theta}^{L}\right\|_{2}>K \varepsilon_{n}^{*}(s) \mid \mathbf{X}_{n}\right)=o(1), \\
\forall \eta>0, \quad \sup _{L \in \Sigma(R, s)} \mathbb{E}_{L} \Pi\left(\left\|\boldsymbol{\theta}+\boldsymbol{\Xi}^{\boldsymbol{\theta}}-\boldsymbol{\theta}^{L}\right\|_{1, \infty}>\eta \mid \mathbf{X}_{n}\right)=o(1) .
\end{gathered}
$$

Proof. From Ghosal and van der Vaart (2007, Lemma 8), because $p_{L}$ is bounded from below (as $\|L\|_{\infty}<\infty$ by assumption), we have that

$$
\begin{aligned}
\left\|L_{\boldsymbol{\theta}}-L\right\|_{2}^{2} & =\int_{[0,1]} \log ^{2}\left(p_{\boldsymbol{\theta}} / p_{L}\right) \\
& \lesssim \int_{[0,1]} p_{L} \log ^{2}\left(p_{\boldsymbol{\theta}} / p_{L}\right) \\
& \lesssim \mathbf{H}\left(P_{\boldsymbol{\theta}}, P_{L}\right)^{2}\left(1+\left\|L_{\boldsymbol{\theta}}-L\right\|_{\infty}^{2}\right) .
\end{aligned}
$$

But, for any $J \in \mathbb{N}$ large enough so that $\Xi_{j, k}=0$ for all $j>J$ and all $k$, we have

$$
\begin{aligned}
\left\|L_{\boldsymbol{\theta}}-L\right\|_{\infty} \lesssim & \sum_{j=0}^{J} \max _{k}\left|\theta_{j, k}+\Xi_{v}^{\boldsymbol{\theta}}-\theta_{j, k}^{L}\right| 2^{j / 2} \\
& +\sum_{j>J} \max _{k}\left|\theta_{j, k}\right| 2^{j / 2}+\sum_{j>J} \max _{k}\left|\theta_{j, k}^{L}\right| 2^{j / 2}
\end{aligned}
$$


By assumption $\sum_{j>J} \max _{k}\left|\theta_{j, k}^{L}\right| 2^{j / 2} \lesssim 2^{-J s}$, and by Proposition $S 4.1$ there is a set $\Theta_{n}$ of posterior mass $1+o_{p}(1)$ such that for any $\boldsymbol{\theta} \in \Theta_{n}$ we have $\sum_{j>J} \max _{k}\left|\theta_{j, k}\right| 2^{j / 2} \lesssim 2^{-J s_{0}} \log (n)$. Hence, whenever $s \geq s_{0}$, for $\boldsymbol{\theta} \in \Theta_{n}$,

$$
\begin{aligned}
\left\|L_{\boldsymbol{\theta}}-L\right\|_{\infty} & \lesssim \sum_{j=0}^{J} \max _{k}\left|\theta_{j, k}+\Xi_{v}^{\boldsymbol{\theta}}-\theta_{j, k}^{L}\right| 2^{j / 2}+2^{-J s_{0}} \log (n)+2^{-J s} \\
& \lesssim 2^{J / 2}\left\|\boldsymbol{\theta}+\boldsymbol{\Xi}^{\boldsymbol{\theta}}-\boldsymbol{\theta}^{L}\right\|_{2}+2^{-J s_{0}} \log (n) \\
& =2^{J / 2}\left\|L_{\boldsymbol{\theta}}-L\right\|_{2}+2^{-J s_{0}} \log (n),
\end{aligned}
$$

where the last line follows by the orthogonality of the wavelet basis. Combining the last display with equation (S4.1) we find that

$$
\left\|L_{\boldsymbol{\theta}}-L\right\|_{2}^{2}\left(1-2^{J} \mathbf{H}\left(P_{\boldsymbol{\theta}}, P_{L}\right)^{2}\right) \lesssim \mathbf{H}\left(P_{\boldsymbol{\theta}}, P_{L}\right)^{2}\left(1+2^{-2 J s_{0}} \log ^{2}(n)\right) .
$$

By equation (S3.1), we can furthermore restrict ourselves to the event such that $\left\{\boldsymbol{\theta}: \mathrm{H}\left(P_{\boldsymbol{\theta}}, P_{L}\right) \lesssim\right.$ $\left.\varepsilon_{n}^{*}(s)\right\}$. Then, it is always possible to choose $J$ sufficiently large so that $2^{-J s_{0}} \log (n)=o(1)$, but small enough so that $2^{J} \varepsilon_{n}^{*}(s)^{2}=o(1)$. Thus $\left\|L_{\boldsymbol{\theta}}-L\right\|_{2}^{2} \lesssim \varepsilon_{n}^{*}(s)^{2}$ on $\left\{\boldsymbol{\theta}: \mathbf{H}\left(P_{\boldsymbol{\theta}}, P_{L}\right) \lesssim \varepsilon_{n}^{*}(s)\right\}$, and by orthogonality of the wavelet basis $\left\|\boldsymbol{\theta}+\boldsymbol{\Xi}^{\boldsymbol{\theta}}-\boldsymbol{\theta}^{L}\right\|_{2}=\left\|L_{\boldsymbol{\theta}}-L\right\|_{2} \lesssim \varepsilon_{n}^{*}(s)$. Moreover, we have proven along the way that on the same event $\left\|\boldsymbol{\theta}+\boldsymbol{\Xi}^{\boldsymbol{\theta}}-\boldsymbol{\theta}^{L}\right\|_{1, \infty} \lesssim 2^{J / 2}\left\|L_{\boldsymbol{\theta}}-L\right\|_{2}+2^{-J s_{0}} \log (n)=$ $o(1)$.

\section{S5. Missing proofs of the main document}

\section{S5.1. Concentration of the posterior on $\mathscr{C}_{*}$}

Proof of Lemma 3.1. We prove the lemma by showing that $\left\|F_{0}^{\boldsymbol{\theta}}\right\|_{\infty}+\left\|\sum_{m \geq 1} F_{m}^{\boldsymbol{\theta}}\right\|_{\infty} \lesssim \| \boldsymbol{\theta}+\boldsymbol{\Xi}^{\boldsymbol{\theta}}-$ $\boldsymbol{\theta}^{L} \|_{1, \infty}$. Then, the conclusion of the lemma will follow from Proposition S4.2. We note that it is enough to show the inequality for $\left\|\boldsymbol{\theta}+\boldsymbol{\Xi}^{\boldsymbol{\theta}}-\boldsymbol{\theta}^{L}\right\|_{1, \infty} \ll 1$ because of Proposition S4.2. For $m \geq$ 1 , we have $F_{m}^{\boldsymbol{\theta}}=\left(\theta_{\psi(m)}-\theta_{\psi(m)}^{L}\right)\left(\varphi_{\psi(m)}-\mathbb{E}_{L}\left[\varphi_{\psi(m)}\right]\right)=\left(\theta_{\psi(m)}+\Xi_{\psi(m)}^{\boldsymbol{\theta}}-\theta_{\psi(m)}^{L}\right)\left(\varphi_{\psi(m)}-\right.$ $\left.\mathbb{E}_{L}\left[\varphi_{\psi(m)}\right]\right)$, by choosing $J_{0}$ sufficiently large so that $\Xi_{\psi(m)}^{\boldsymbol{\theta}}=0$ whenever $m \neq 0$. It follows by Proposition 7.1-(5),

$$
\begin{aligned}
\left\|\sum_{m \geq 1} F_{m}^{\boldsymbol{\theta}}\right\|_{\infty} & \leq \sup _{x} \sum_{m \geq 1}\left|\theta_{\psi(m)}^{\boldsymbol{\theta}}+\Xi_{\psi(m)}^{\boldsymbol{\theta}}-\theta_{\psi(m)}^{L} \| \varphi_{\psi(m)}(x)-\mathbb{E}_{L}\left[\varphi_{\psi(m)}\right]\right| \\
& \leq \sum_{j>J_{0}} \max _{k}\left|\theta_{j, k}^{\boldsymbol{\theta}}+\Xi_{j, k}^{\boldsymbol{\theta}}-\theta_{j, k}^{L}\right| \sup _{x} \sum_{k}\left|\varphi_{j, k}(x)-\mathbb{E}_{L}\left[\varphi_{j, k}\right]\right| \\
& \lesssim \sum_{j>J_{0}} \max _{k}\left|\theta_{j, k}^{\boldsymbol{\theta}}+\Xi_{j, k}^{\boldsymbol{\theta}}-\theta_{j, k}^{L}\right| 2^{j / 2} \\
& \leq\left\|\boldsymbol{\theta}+\boldsymbol{\Xi}^{\boldsymbol{\theta}}-\boldsymbol{\theta}^{L}\right\|_{1, \infty} .
\end{aligned}
$$


So it remains to show that $\left\|F_{0}^{\boldsymbol{\theta}}\right\|_{\infty}$ is also bounded by the same quantity. Remark that $\mathbb{E}_{L}\left[\exp \left\{L_{\boldsymbol{\theta}}-\right.\right.$ $L\}]=1$, thus

$$
1=\mathbb{E}_{L}\left[e^{L_{\boldsymbol{\theta}}-L}\right]=1+\mathbb{E}_{L}\left[L_{\boldsymbol{\theta}}-L\right]+\frac{1}{2} \mathbb{E}_{L}\left[\left(L_{\boldsymbol{\theta}}-L\right)^{2}\right] \cdot O\left(e^{\left\|L_{\boldsymbol{\theta}}-L\right\|_{\infty}}\right) .
$$

But $\mathbb{E}_{L}\left[L_{\boldsymbol{\theta}}-L\right]=\xi^{\boldsymbol{\theta}}+\sum_{(j, k) \in \mathcal{V}}\left(\theta_{j, k}-\theta_{j, k}^{L}\right) \mathbb{E}_{L}\left[\varphi_{j, k}\right]$ and $\left\|L_{\boldsymbol{\theta}}-L\right\|_{\infty} \lesssim\left\|\boldsymbol{\theta}+\boldsymbol{\Xi}^{\boldsymbol{\theta}}-\boldsymbol{\theta}^{L}\right\|_{1, \infty} \ll 1$ by assumption. Therefore, we have shown

$$
\xi^{\boldsymbol{\theta}}=-\sum_{(j, k) \in \mathcal{V}}\left(\theta_{j, k}-\theta_{j, k}^{L}\right) \mathbb{E}_{L}\left[\varphi_{j, k}\right]+O\left(\left\|L_{\boldsymbol{\theta}}-L\right\|_{2}^{2}\right) .
$$

Since for $J_{0}$ taken large enough we have $\xi^{\boldsymbol{\theta}}=\sum_{(j, k) \in B_{0}} \Xi_{j, k} \varphi_{j, k}$, we deduce that

$$
\begin{aligned}
F_{0} & =\sum_{(j, k) \in B_{0}}\left(\theta_{j, k}-\theta_{j, k}^{L}\right)\left(\varphi_{j, k}-\mathbb{E}_{L}\left[\varphi_{j, k}\right]\right) \\
& =\xi^{\boldsymbol{\theta}}+\sum_{(j, k) \in B_{0}}\left(\theta_{j, k}-\theta_{j, k}^{L}\right) \varphi_{j, k}+\sum_{(j, k) \notin B_{0}}\left(\theta_{j, k}-\theta_{j, k}^{L}\right) \mathbb{E}_{L}\left[\varphi_{j, k}\right]+O\left(\left\|L_{\boldsymbol{\theta}}-L\right\|_{2}^{2}\right) \\
& =\sum_{(j, k) \in B_{0}}\left(\theta_{j, k}+\Xi_{j, k}^{\boldsymbol{\theta}}-\theta_{j, k}^{L}\right) \varphi_{j, k}+\sum_{(j, k) \notin B_{0}}\left(\theta_{j, k}+\Xi_{j, k}^{\boldsymbol{\theta}}-\theta_{j, k}^{L}\right) \mathbb{E}_{L}\left[\varphi_{j, k}\right]+O\left(\left\|L_{\boldsymbol{\theta}}-L\right\|_{2}^{2}\right) .
\end{aligned}
$$

Now we remark that all the terms involved in the last display are bounded in absolute value by $\| \boldsymbol{\theta}+$ $\boldsymbol{\Xi}^{\boldsymbol{\theta}}-\boldsymbol{\theta}^{L} \|_{1, \infty}$, and so is $\left\|F_{0}\right\|_{\infty}$.

\section{S5.2. Proofs related to the control of the covariance terms}

Proof of Lemma 6.4. We first establish that $\left\|S_{\overline{\boldsymbol{F}}_{I}}\right\|_{\infty}$ can be made arbitrary small when $\overline{\boldsymbol{F}}_{I} \in \mathcal{N}_{I}$ by taking $K_{1}$ large enough in Assumption 1.

Proposition S5.1. Suppose Assumption 1 is satisfied with $K_{1}>0$ sufficiently large. Then there is a universal constant $C>0$ such that $\left\|S_{\overline{\boldsymbol{F}}_{I}}\right\|_{\infty} \leq C \log \left(\Gamma \varepsilon_{n}^{*}(s) / \gamma\right) \Gamma \varepsilon_{n}^{*}(s)$ for all $\overline{\boldsymbol{F}}_{I} \in \mathcal{N}_{I}$ and all $I \subseteq \mathbb{Z}_{+}$.

In view of the last proposition, we now assume that $K_{1}$ is taken large enough so that $\left\|S_{\overline{\boldsymbol{F}}_{I}}\right\|_{\infty} \ll \delta$. Since $\mathbb{E}_{L}\left[S_{\boldsymbol{F}_{I}}\right]=\mathbb{E}_{L}\left[S_{\overline{\boldsymbol{F}}_{I}}\right]=0$, we have

$$
\mathrm{C}\left(S_{\boldsymbol{F}_{I}}, S_{\overline{\boldsymbol{F}}_{I}}\right)=\log \mathbb{E}_{L}\left[e^{S_{\boldsymbol{F}_{I}}-\log \mathbb{E}_{L}\left[\exp \left(S_{\boldsymbol{F}_{I}}\right)\right]} e^{S_{\overline{\boldsymbol{F}}_{I}}-\log \mathbb{E}_{L}\left[\exp \left(S_{\overline{\boldsymbol{F}}_{I}}\right)\right]}\right] .
$$

We remark that $\mathbb{E}_{L}\left[e^{\left.S_{\boldsymbol{F}_{I}}\right]}=\mathbb{E}_{L}\left[1+S_{\boldsymbol{F}_{I}}+\frac{1}{2} S_{\boldsymbol{F}_{I}}^{2} e^{O\left(\left\|S_{\boldsymbol{F}_{I}}\right\|_{\infty}\right)}\right]=1+\frac{1}{2} \mathbb{E}_{L}\left[S_{\boldsymbol{F}_{I}}^{2}\right] e^{O(\delta)}\right.$. This implies that $-\log \mathbb{E}_{L}\left[e^{\left.S_{\boldsymbol{F}_{I}}\right]}=-\frac{1}{2} \mathbb{E}_{L}\left[S_{\boldsymbol{F}_{I}}^{2}\right] e^{O(\delta)}\right.$ as well, and $e^{S_{\boldsymbol{F}_{I}}-\log \mathbb{E}_{L}\left[\exp \left(S_{\boldsymbol{F}_{I}}\right)\right]}=1+S_{\boldsymbol{F}_{I}}-$ $\frac{1}{2} \mathbb{E}_{L}\left[S_{\boldsymbol{F}_{I}}^{2}\right] e^{O(\delta)}+\frac{1}{2} S_{\boldsymbol{F}_{I}}^{2} e^{O(\delta)}$. Also $\mathbb{E}_{L}\left[e^{S_{\overline{\boldsymbol{F}}_{I}}-\log \mathbb{E}_{L}\left[\exp \left(S_{\overline{\boldsymbol{F}}_{I}}\right)\right]}\right]=1$, and thus

$$
\begin{aligned}
e^{\mathrm{C}\left(S_{\boldsymbol{F}_{I}}, S_{\overline{\boldsymbol{F}}_{I}}\right)} & =\mathbb{E}_{L}\left[\left(1+S_{\boldsymbol{F}_{I}}+\frac{1}{2} S_{\boldsymbol{F}_{I}}^{2} e^{O(\delta)}-\frac{1}{2} \mathbb{E}_{L}\left[S_{\boldsymbol{F}_{I}}^{2}\right] e^{O(\delta)}\right) e^{S_{\overline{\boldsymbol{F}}_{I}}-\log \mathbb{E}_{L}\left[\exp \left(S_{\overline{\boldsymbol{F}}_{I}}\right)\right]}\right] \\
& =1+\mathbb{E}_{L}\left[S_{\boldsymbol{F}_{I}} e^{S_{\overline{\boldsymbol{F}}_{I}}-\log \mathbb{E}_{L}\left[\exp \left(S_{\overline{\boldsymbol{F}}_{I}}\right)\right]}\right]+\frac{1}{2} \mathbb{E}_{L}\left[S_{\boldsymbol{F}_{I}}^{2}\right] e^{O(\delta)}-\frac{1}{2} \mathbb{E}_{L}\left[S_{\boldsymbol{F}_{I}}^{2}\right] e^{O(\delta)}
\end{aligned}
$$




$$
=1+\mathbb{E}_{L}\left[S_{\boldsymbol{F}_{I}} e^{S_{\overline{\boldsymbol{F}}_{I}}-\log \mathbb{E}_{L}\left[\exp \left(S_{\overline{\boldsymbol{F}}_{I}}\right)\right]}\right]+O\left(\delta \mathbb{E}_{L}\left[S_{\boldsymbol{F}_{I}}^{2}\right]\right) .
$$

Since $\left\|S_{\overline{\boldsymbol{F}}_{I}}\right\|_{\infty} \ll \delta$,

$$
\mathbb{E}_{L}\left[S_{\boldsymbol{F}_{I}} e^{S_{\overline{\boldsymbol{F}}_{I}}-\log \mathbb{E}_{L}\left[\exp \left(S_{\overline{\boldsymbol{F}}_{I}}\right)\right]}\right]^{2} \leq\left\|S_{\boldsymbol{F}_{I}}\right\|_{\infty} e^{O(\delta)}\left|\mathbb{E}_{L}\left[S_{\boldsymbol{F}_{I}} e^{S_{\overline{\boldsymbol{F}}_{I}}-\log \mathbb{E}_{L}\left[\exp \left(S_{\overline{\boldsymbol{F}}_{I}}\right)\right]}\right]\right| .
$$

Therefore,

$$
\begin{aligned}
& \mathrm{C}\left(S_{\boldsymbol{F}_{I}}, S_{\overline{\boldsymbol{F}}_{I}}\right)=(1+O(\delta)) \mathbb{E}_{L}\left[S_{\boldsymbol{F}_{I}} e^{S_{\overline{\boldsymbol{F}}_{I}}-\log \mathbb{E}_{L}\left[\exp \left(S_{\overline{\boldsymbol{F}}_{I}}\right)\right]}\right]+O\left(\delta \mathbb{E}_{L}\left[S_{\boldsymbol{F}_{I}}^{2}\right]\right) \\
& =(1+O(\delta)) \frac{\mathbb{E}_{L}\left[S_{\boldsymbol{F}_{I}}\left(e^{\left.S_{\overline{\boldsymbol{F}}_{I}}-1\right)}\right]\right.}{\mathbb{E}_{L}\left[e^{\left.S_{\overline{\boldsymbol{F}}_{I}}\right]}\right.}+O\left(\delta \mathbb{E}_{L}\left[S_{\boldsymbol{F}_{I}}^{2}\right]\right) \\
& =O\left(\mathbb { E } _ { L } \left[S_{\boldsymbol{F}_{I}}\left(e^{\left.\left.S_{\overline{\boldsymbol{F}}_{I}}-1\right)\right]}\right)+O\left(\delta \mathbb{E}_{L}\left[S_{\boldsymbol{F}_{I}}^{2}\right]\right) .\right.\right.
\end{aligned}
$$

To prove the lemma, it is enough to obtain sharp estimates on $\mathbb{E}_{L}\left[S_{\boldsymbol{F}_{I}}\left(e^{S_{\overline{\boldsymbol{F}}_{I}}}-1\right)\right]$. To this end, we introduce the function $A: \mathbb{R} \rightarrow \mathbb{R}$ such that $A(x)=\left(e^{x}-1\right) / x$, and we define the following "covariance" matrix

$$
\Sigma_{j, k, j^{\prime}, k^{\prime}}^{\overline{\boldsymbol{F}}_{I}}:=\mathbb{E}_{L}\left[\left(\varphi_{j, k}-\mathbb{E}_{L}\left[\varphi_{j, k}\right]\right)\left(\varphi_{j^{\prime}, k^{\prime}}-\mathbb{E}_{L}\left[\varphi_{j^{\prime}, k^{\prime}}\right]\right) A\left(S_{\overline{\boldsymbol{F}}_{I}}\right)\right] .
$$

The following proposition provides the necessary estimates on $\Sigma^{\overline{\boldsymbol{F}}_{I}}$ that we require to control $\mathrm{C}\left(S_{\boldsymbol{F}_{I}}, S_{\overline{\boldsymbol{F}}_{I}}\right)$.

Proposition S5.2. Let $\left\|S_{\overline{\boldsymbol{F}}_{I}}\right\|_{\infty} \leq 1$ and let $\tilde{s}=s$ if $s<1$ and $\tilde{s}$ arbitrary in $(1 / 2,1)$ if $s \geq 1$. Then, the following are true for $n$ large enough (but not depending on I)

1. There exists a constant $C>0$ depending only on $(R, s)$ and the wavelet basis, such that for all $\left(j^{\prime}, k^{\prime}\right) \neq(j, k)$,

$$
\left|\Sigma_{j, k, j^{\prime}, k^{\prime}}^{\overline{\boldsymbol{F}}_{I}}\right| \leq C\left(1+\left\|S_{\overline{\boldsymbol{F}}_{I}}\right\|_{\infty, \infty, \tilde{s}}\right)\left(2^{-\left(j \vee j^{\prime}\right) \tilde{s}} \int\left|\varphi_{j, k} \varphi_{j^{\prime}, k^{\prime}}\right|+2^{-j(\tilde{s}+1 / 2)} 2^{-j^{\prime}(\tilde{s}+1 / 2)}\right) .
$$

2. There exists a constant $C>0$ depending only on $(R, s)$ and the wavelet basis, such that for any

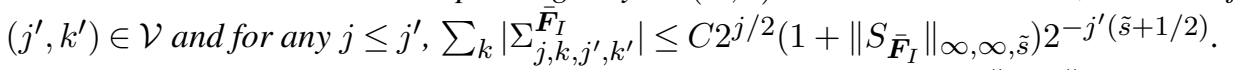

3. Suppose Assumption 1 is satisfied for $K_{0}$ and $K_{1}$ large enough. Then $\left\|S_{\overline{\boldsymbol{F}}_{I}}\right\|_{\infty, \infty, \tilde{s}} \leq \gamma$ for all $I \subseteq \mathbb{Z}_{+}$.

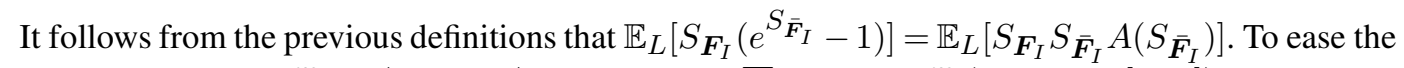
notations, we define $f_{j, k}^{m}:=\left\langle F_{m}, \varphi_{j, k}\right\rangle$, so that $F_{m}=\sum_{(j, k) \in B_{m}} f_{j, k}^{m}\left(\varphi_{j, k}-\mathbb{E}_{L}\left[\varphi_{j, k}\right]\right)$, by Proposition 7.1-(1). Hence, we can rewrite

$$
\begin{aligned}
\mathbb{E}_{L}\left[S _ { \boldsymbol { F } _ { I } } \left(e^{\left.\left.S_{\overline{\boldsymbol{F}}_{I}}-1\right)\right]=}\right.\right. & \sum_{m \in I^{c}} \sum_{(j, k) \in B_{0}} \sum_{\left(j^{\prime}, k^{\prime}\right) \in B_{m}} f_{j, k}^{0} f_{j^{\prime}, k^{\prime}}^{m} \Sigma_{j, k, j^{\prime}, k^{\prime}}^{\overline{\boldsymbol{F}}_{I}} \mathbf{1}_{0 \in I} \\
& +\sum_{m \in I} \sum_{(j, k) \in B_{m}} \sum_{\left(j^{\prime}, k^{\prime}\right) \in B_{0}} f_{j^{\prime}, k^{\prime}}^{0} f_{j, k}^{m} \Sigma_{j, k, j^{\prime}, k^{\prime}}^{\overline{\boldsymbol{F}}_{I}} \mathbf{1}_{0 \notin I} \\
& +\sum_{m \in I} \sum_{m^{\prime} \in I^{c}} \sum_{(j, k) \in B_{m}} \sum_{\left(j^{\prime}, k^{\prime}\right) \in B_{m^{\prime}}} f_{j, k}^{m} f_{j^{\prime}, k^{\prime}}^{m^{\prime}} \Sigma_{j, k, j^{\prime}, k^{\prime}} \mathbf{1}_{m \neq 0} \mathbf{1}_{m^{\prime} \neq 0} .
\end{aligned}
$$


We finish the proof by giving a bound on the terms

$$
\begin{aligned}
R_{1} & :=\sum_{m \in I^{c}} \sum_{(j, k) \in B_{0}} \sum_{\left(j^{\prime}, k^{\prime}\right) \in B_{m}}\left|f_{j, k}^{0} \| f_{j^{\prime}, k^{\prime}}^{m}\right|\left|\Sigma_{j, k, j^{\prime}, k^{\prime}}^{\overline{\boldsymbol{F}}_{I}}\right| \mathbf{1}_{0 \in I}, \\
R_{2} & :=\sum_{m \in I} \sum_{(j, k) \in B_{m}} \sum_{\left(j^{\prime}, k^{\prime}\right) \in B_{0}}\left|f_{j^{\prime}, k^{\prime}}^{0}\right|\left|f_{j, k}^{m}\right|\left|\Sigma_{j, k, j^{\prime}, k^{\prime}}\right| \mathbf{1}_{0 \notin I}, \\
R_{3} & :=\sum_{m \in I} \sum_{m^{\prime} \in I^{c}} \sum_{(j, k) \in B_{m}} \sum_{\left(j^{\prime}, k^{\prime}\right) \in B_{m^{\prime}}}\left|f_{j, k}^{m}\right|\left|f_{j^{\prime}, k^{\prime}}^{m^{\prime}}\right|\left|\Sigma_{j, k, j^{\prime}, k^{\prime}}\right| \mathbf{1}_{m \neq 0} \mathbf{1}_{m^{\prime} \neq 0} .
\end{aligned}
$$

We bound each of the terms above in the next paragraphs. For each term, the bound depends on whether we are in the scenario $s>1 / 2$ or not. An important quantity that shows up everywhere is $f^{m, *}:=$ $\sup _{(j, k) \in B_{m}}\left|\left\langle F_{m}, \varphi_{j, k}\right\rangle\right|$. We remark that by construction when $m \notin I$

$$
f^{m, *}:=\sup _{(j, k) \in B_{m}}\left|\left\langle F_{m}, \varphi_{v}\right\rangle\right| \leq\left\|F_{m}\right\|_{2} \leq H_{I}(m) .
$$

Bound on $R_{1}$ : case $s>1 / 2$. If $0 \notin I$ this term is obviously equal to zero. Thus we now assume that $0 \in I$. Then,

$$
\begin{aligned}
R_{1} & \leq f^{0, *} \sum_{m \in I^{c}} f^{m, *} \sum_{\left(j^{\prime}, k^{\prime}\right) \in B_{m}} \sum_{j=0}^{J_{0}} \sum_{k}\left|\Sigma_{j, k, j^{\prime}, k^{\prime}}^{\overline{\boldsymbol{F}}_{I}}\right| \\
& \lesssim f^{0, *}(1 \vee \gamma) 2^{J_{0} / 2} \sum_{m \in I^{c}} f^{m, *} \sum_{\left(j^{\prime}, k^{\prime}\right) \in B_{m}} 2^{-j^{\prime}(\tilde{s}+1 / 2)} \\
& \lesssim f^{0, *}(1 \vee \gamma) 2^{J_{0} / 2} \sum_{m \in I^{c}} f^{m, *} 2^{-J_{m}(\tilde{s}+1 / 2)}
\end{aligned}
$$

where the second line follows by Proposition S5.2, and the last because $0 \in I$ so for any $m \in I^{c}$ we have $\left|B_{m}\right|=1$ and $(j, k) \in B_{m} \Longrightarrow j=J_{m}$. So by equation (S5.1),

$$
\begin{aligned}
& \sum_{m \in I^{c}} f^{m, *} 2^{-J_{m}(\tilde{s}+1 / 2) \leq} \leq \sum_{m \in I^{c}} H_{I}(m) 2^{-J_{m}(\tilde{s}+1 / 2)} \\
& \leq \Gamma \xi^{-1} \sum_{j=J_{0}+1}^{j_{n}} \sup _{m: J_{m}=j}\left\{\rho_{m}\right\}\left|\left\{m \in I^{c}: J_{m}=j\right\}\right| 2^{-j(\tilde{s}+1 / 2)} \\
& \quad+\sum_{j>j_{n}} \gamma 2^{-j(s+\tilde{s}+1)}\left|\left\{m \in I^{c}: J_{m}=j\right\}\right| \\
& \leq \Gamma \xi^{-1} \sum_{j>J_{0}} \sup _{m: J_{m}=j}\left\{\rho_{m}\right\} 2^{-j(\tilde{s}-1 / 2)}+\gamma 2^{-j_{n}(s+\tilde{s})},
\end{aligned}
$$

where the last line follows because $\left|\left\{m \in I^{c}: J_{m}=j\right\}\right| \lesssim 2^{j}$ for $j>J_{0}$ as there are no more than $\lesssim 2^{j}$ at each level. We deduce that,

$$
R_{1} \lesssim f^{0, *} \Gamma \xi^{-1}(1 \vee \gamma) 2^{J_{0} / 2} \sum_{j>J_{0}} 2^{-j(\tilde{s}-1 / 2)} \sup _{m: J_{m}=j}\left\{\rho_{m}\right\}+f^{0, *}(1 \vee \gamma) 2^{J_{0} / 2} \gamma 2^{-j_{n}(s+\tilde{s})}
$$




$$
\lesssim\left\|F_{0}\right\|_{2} \sqrt{\frac{\log (n)}{n}} \cdot \frac{\Gamma(1 \vee \gamma)}{\xi} 2^{J_{0}(1-\tilde{s})}+\left\|F_{0}\right\|_{2} \sqrt{\frac{\log (n)}{n}} \cdot 2^{J_{0} / 2} \Gamma(1 \vee \gamma) 2^{-j_{n}(\tilde{s}-1 / 2)}
$$

because $\rho_{m}=\sqrt{\log (n) / n}, \tilde{s}>1 / 2$, because $f^{0, *} \leq\left\|F_{0}\right\|_{2}$, and because of equation (3.2). By choosing $K_{1}, K_{2}>0$ in Assumption 1 sufficiently large we obtain that $R_{1} \ll \delta \Gamma \sqrt{\log (n) / n}\left\|F_{0}\right\|_{2} \mathbf{1}_{0 \in I}$.

Bound on $R_{1}$ : case $0<s \leq 1 / 2$. The equation (S5.2) remains true in this case, but this time we have,

$$
\begin{aligned}
\sum_{j>J_{0}} 2^{-j(\tilde{s}-1 / 2)} \sup _{m: J_{m}=j}\left\{\rho_{m}\right\} & \leq \sum_{j>J_{0}} 2^{-j(s-1 / 2)} \sup _{m: J_{m}=j}\left\{2^{-J_{m} / 2} \varepsilon_{n}^{*}(s)\right\} \\
& =\varepsilon_{n}^{*}(s) \sum_{j>J_{0}} 2^{-j s} \\
& \lesssim \varepsilon_{n}^{*}(s) 2^{-J_{0} s}
\end{aligned}
$$

and, by equation (3.2),

$$
\gamma 2^{-j_{n}(s+\tilde{s})}=\gamma 2^{-2 j_{n} s} \lesssim \frac{\Gamma^{2} \varepsilon_{n}^{*}(s)^{2}}{\gamma}
$$

Therefore,

$$
R_{1} \lesssim\left\|F_{0}\right\|_{2} \Gamma 2^{-J_{0} / 2} \varepsilon_{n}^{*}(s)\left(\frac{1 \vee \gamma}{\xi} 2^{J_{0}(1-s)}+2^{J_{0}} \frac{\Gamma(1 \vee \gamma)}{\gamma} \varepsilon_{n}^{*}(s)\right)
$$

So $R_{1} \ll \delta \Gamma 2^{-J_{0} / 2} \varepsilon_{n}^{*}(s)\left\|F_{0}\right\|_{2} \mathbf{1}_{0 \in I}$ by choosing $K_{1}, K_{2}>0$ in Assumption 1 sufficiently large.

Bound on $R_{2}$ : case $s>1 / 2 . \quad R_{2}$ is obviously equal to zero of $0 \in I$, hence we assume now that $0 \notin I$. Then with the same arguments as for the first term

$$
\begin{aligned}
R_{2} & \leq f^{0, *} \sum_{m \in I} \sum_{(j, k) \in B_{m}}\left|f_{j, k}^{m}\right| \sum_{j^{\prime}=0}^{J_{0}} \sum_{k^{\prime}}\left|\Sigma_{j, k, j^{\prime}, k^{\prime}}^{\overline{\boldsymbol{F}}_{I}}\right| \\
& \lesssim(1 \vee \gamma) 2^{J_{0} / 2} f^{0, *} \sum_{m \in I} \sum_{(j, k) \in B_{m}}\left|f_{j, k}^{m}\right| 2^{-J_{m}(\tilde{s}+1 / 2)} \\
& \lesssim(1 \vee \gamma) 2^{J_{0} / 2} H_{I}(0) \sum_{m \in I}\left\|F_{m}\right\|_{2} 2^{-J_{m}(\tilde{s}+1 / 2)},
\end{aligned}
$$

where the second line follows by Proposition S5.2-(2) and because $(j, k) \in B_{m} \Longrightarrow j=J_{m}$ when $m \neq 0$ (recall $0 \notin I$ ), the third last line follows because $0 \notin I$ so $B_{m}$ is a singleton and also because $f^{0, *} \leq H_{I}(0)$ when $0 \notin I$. We decompose the sum in the rhs equation (S5.4) into the sum of the following two terms

$$
\begin{aligned}
S_{1} & :=\sum_{m \in I}\left\|F_{m}\right\|_{2} 2^{-J_{m}(\tilde{s}+1 / 2)} \mathbf{1}_{J_{m} \leq j_{n}} \\
S_{2} & :=\sum_{m \in I}\left\|F_{m}\right\|_{2} 2^{-J_{m}(\tilde{s}+1 / 2)} \mathbf{1}_{J_{m}>j_{n}}
\end{aligned}
$$


Then, by Cauchy-Schwarz',

$$
S_{1} \leq\left\{\sum_{m \in I}\left\|F_{m}\right\|_{2}^{2} \mathbf{1}_{J_{m} \leq j_{n}}\right\}^{1 / 2}\left\{\sum_{m \in I} 2^{-J_{m}(2 \tilde{s}+1)} \mathbf{1}_{J_{m} \leq j_{n}}\right\}^{1 / 2} .
$$

But, since $0 \notin I$ we have $m \in I \Rightarrow J_{m}>J_{0}$, and since there are $\lesssim 2^{j}$ wavelets at each level $j$, we deduce that

$$
\sum_{m \in I} 2^{-J_{m}(2 \tilde{s}+1)} \leq \sum_{j>J_{0}}\left|\left\{m: J_{m}=j\right\}\right| 2^{-j(2 \tilde{s}+1)} \lesssim \sum_{j>J_{0}} 2^{-2 j \tilde{s}} \lesssim 2^{-2 J_{0} \tilde{s}} .
$$

Hence,

$$
S_{1} \lesssim 2^{-J_{0} \tilde{s}}\left\{\sum_{m \in I}\left\|F_{m}\right\|_{2}^{2} \mathbf{1}_{J_{m} \leq j_{n}}\right\}^{1 / 2}
$$

On the other hand,

$$
\begin{aligned}
S_{2} & \leq\left\{\sum_{m \in I}\left\|F_{m}\right\|_{2}^{2} \mathbf{1}_{J_{m}>j_{n}}\right\}^{1 / 2}\left\{\sum_{m \in I} 2^{-J_{m}(2 \tilde{s}+1)} \mathbf{1}_{J_{m}>j_{n}}\right\}^{1 / 2} \\
& \lesssim\left\{\sum_{m \in I}\left\|F_{m}\right\|_{2}^{2} \mathbf{1}_{J_{m}>j_{n}}\right\}^{1 / 2}\left\{\sum_{j>j_{n}} 2^{-2 j \tilde{s}}\right\}^{1 / 2} \\
& \lesssim 2^{-j_{n} \tilde{s}}\left\{\sum_{m \in I}\left\|F_{m}\right\|_{2}^{2} \mathbf{1}_{J_{m}>j_{n}}\right\}^{1 / 2} .
\end{aligned}
$$

Since $H_{I}(0)=\Gamma \sqrt{\log (n) / n}$ in this case,

$$
\begin{aligned}
R_{2} \lesssim \Gamma(1 \vee \gamma) 2^{J_{0}(1 / 2-\tilde{s})} \sqrt{\frac{\log (n)}{n}} & \left\{\sum_{m \in I}\left\|F_{m}\right\|_{2}^{2} \mathbf{1}_{J_{m} \leq j_{n}}\right\}^{1 / 2} \\
& +\Gamma(1 \vee \gamma) 2^{J_{0} / 2} \cdot 2^{-j_{n} \tilde{s}} \sqrt{\frac{\log (n)}{n}}\left\{\sum_{m \in I}\left\|F_{m}\right\|_{2}^{2} \mathbf{1}_{J_{m}>j_{n}}\right\}^{1 / 2} .
\end{aligned}
$$

Note that in this case $\tilde{s}>1 / 2$, so by choosing the constants $K_{0}$ and $K_{1}$ sufficiently large, we obtain that (note that $\xi^{-\mathbf{1}_{0 \in I}}=1$ here)

$$
R_{2} \ll \delta \Gamma \xi^{-\mathbf{1}_{0 \in I}} \sqrt{\frac{\log (n)}{n}}\left\{\sum_{m \in I}\left\|F_{m}\right\|_{2}^{2} \mathbf{1}_{J_{m} \leq j_{n}}\right\}^{1 / 2}+\frac{\delta}{\sqrt{n}}\left\{\sum_{m \in I}\left\|F_{m}\right\|_{2}^{2} \mathbf{1}_{J_{m}>j_{n}}\right\}^{1 / 2} .
$$

Bound on $R_{2}$ : case $0<s \leq 1 / 2$. We note that the equation equation (S5.4) and the decomposition of equations (S5.5) and (S5.6) remain true in this case. We then have,

$$
S_{1} \leq\left\{\sum_{m \in I}\left\|F_{m}\right\|_{2}^{2} \mathbf{1}_{J_{m} \leq j_{n}}\right\}^{1 / 2}\left\{\sum_{m \in I} 2^{-J_{m}(2 s+1)} \mathbf{1}_{J_{m} \leq j_{n}}\right\}^{1 / 2}
$$


Since $0 \notin I$ we have that $m \in I \Longrightarrow J_{m} \geq J_{1}>J_{0}$, so that the previous is in fact bounded by

$$
S_{1} \leq 2^{-J_{0} s}\left\{\sum_{m \in I}\left\|F_{m}\right\|_{2}^{2} \mathbf{1}_{J_{m} \leq j_{n}}\right\}^{1 / 2}\left\{\sum_{m \in I} 2^{-J_{m}} \mathbf{1}_{J_{m} \leq j_{n}}\right\}^{1 / 2} .
$$

On the other hand,

$$
S_{2} \leq\left\{\sum_{m \in I}\left\|F_{m}\right\|_{2}^{2} \mathbf{1}_{J_{m}>j_{n}}\right\}^{1 / 2}\left\{\sum_{m \in I} 2^{-J_{m}(2 s+1)} \mathbf{1}_{J_{m}>j_{n}}\right\}^{1 / 2} .
$$

Since $H_{I}(0)=\Gamma 2^{-J_{0} / 2} \varepsilon_{n}^{*}(s)$ in this case,

$$
\begin{aligned}
R_{2} \lesssim \Gamma(1 \vee \gamma) \cdot 2^{-J_{0} s}\left\{\sum_{m \in I}\left\|F_{m}\right\|_{2}^{2} \mathbf{1}_{J_{m} \leq j_{n}}\right\}^{1 / 2}\left\{\varepsilon_{n}^{*}(s)^{2} \sum_{m \in I} 2^{-J_{m}} \mathbf{1}_{J_{m} \leq j_{n}}\right\}^{1 / 2} \\
+\Gamma(1 \vee \gamma) \varepsilon_{n}^{*}(s) \cdot\left\{\sum_{m \in I}\left\|F_{m}\right\|_{2}^{2} \mathbf{1}_{J_{m}>j_{n}}\right\}^{1 / 2}\left\{\sum_{m \in I} 2^{-J_{m}(2 s+1)} \mathbf{1}_{J_{m}>j_{n}}\right\}^{1 / 2} .
\end{aligned}
$$

So by choosing $K_{0}$ and $K_{1}$ large enough,

$$
\begin{aligned}
R_{2} \ll \delta \Gamma \xi^{-\mathbf{1}_{0 \in I}}\left\{\sum_{m \in I}\left\|F_{m}\right\|_{2}^{2} \mathbf{1}_{J_{m} \leq j_{n}}\right\}^{1 / 2}\left\{\varepsilon_{n}^{*}(s)^{2} \sum_{m \in I} 2^{-J_{m}} \mathbf{1}_{J_{m} \leq j_{n}}\right\}^{1 / 2} \\
+\delta \gamma\left\{\sum_{m \in I}\left\|F_{m}\right\|_{2}^{2} \mathbf{1}_{J_{m}>j_{n}}\right\}^{1 / 2}\left\{\sum_{m \in I} 2^{-J_{m}(2 s+1)} \mathbf{1}_{J_{m}>j_{n}}\right\}^{1 / 2} .
\end{aligned}
$$

Bound on $R_{3}$ : case $s>1 / 2$. We start with the following estimate (using equation (S5.1))

$$
\begin{aligned}
\sum_{m^{\prime} \in I^{c}} \sum_{\left(j^{\prime}, k^{\prime}\right) \in B_{m^{\prime}}}\left|f_{j^{\prime}, k^{\prime}}^{m^{\prime}}\right|\left|\Sigma_{j, k, j^{\prime}, k^{\prime}}\right| \mathbf{1}_{m^{\prime} \neq 0} & \overline{\boldsymbol{F}}_{I} \\
& \lesssim \sum_{j^{\prime \prime}>J_{0}} \sum_{m^{\prime} \in I^{c}} \mathbf{1}_{J_{m^{\prime}}=j^{\prime \prime}} \sum_{\left(j^{\prime}, k^{\prime}\right) \in B_{m^{\prime}}}\left|f_{j^{\prime}, k^{\prime}}^{m^{\prime}}\right| \cdot\left|\Sigma_{j, k, j^{\prime}, k^{\prime}}\right| \mathbf{1}_{m^{\prime} \neq 0} \\
& \leq \sum_{j^{\prime \prime}>J_{0}} \sup _{m^{\prime}: J_{m^{\prime}}=j^{\prime \prime}}\left\{H_{I}\left(m^{\prime}\right)\right\} \sum_{m^{\prime} \in I^{c}} \mathbf{1}_{J_{m^{\prime}}=j^{\prime \prime}} \sum_{\left(j^{\prime}, k^{\prime}\right) \in B_{m^{\prime}}}\left|\Sigma_{j, k, j^{\prime}, k^{\prime}}\right| \mathbf{1}_{m^{\prime} \neq 0} \\
& \leq \sum_{j^{\prime}>J_{0}} \sup _{m^{\prime}: J_{m^{\prime}}=j^{\prime}}\left\{H_{I}\left(m^{\prime}\right)\right\} \sum_{k^{\prime}}\left|\Sigma_{j, k, j^{\prime}, k^{\prime}}\right|
\end{aligned}
$$

where the last line follows because for $m^{\prime} \neq 0$ we have $\left(j^{\prime}, k^{\prime}\right) \in B_{m} \Longrightarrow j^{\prime}=J_{m^{\prime}}$ and $B_{m^{\prime}}$ is a singleton. By Proposition S5.2-(1,3), because there are no more than $\lesssim 2^{j}$ wavelets at each level $j \geq 0$, and because $\sup _{x} \sum_{k}\left|\varphi_{j, k}(x)\right| \lesssim 2^{j / 2}$ (see Proposition 7.1-(5)), we obtain

$$
\sum_{k^{\prime}}\left|\sum_{j, k, j^{\prime}, k^{\prime}}^{\overline{\boldsymbol{F}}_{I}}\right| \lesssim(1 \vee \gamma)\left(2^{-\left(j \vee j^{\prime}\right) \tilde{s}} \int\left|\varphi_{j, k}\right| \sum_{k^{\prime}}\left|\varphi_{j^{\prime}, k^{\prime}}\right|+2^{-j(\tilde{s}+1 / 2)} 2^{-j^{\prime}(\tilde{s}+1 / 2)} 2^{j^{\prime}}\right)
$$




$$
\begin{aligned}
& \lesssim(1 \vee \gamma) 2^{-j / 2} 2^{j^{\prime} / 2}\left(2^{-\left(j \vee j^{\prime}\right) \tilde{s}}+2^{-j \tilde{s}} 2^{-j^{\prime} \tilde{s}}\right) \\
& \lesssim(1 \vee \gamma) 2^{-j / 2} 2^{j^{\prime} / 2} 2^{-\left(j \vee j^{\prime}\right) \tilde{s}}
\end{aligned}
$$

Therefore,

$$
\begin{aligned}
R_{3} & \lesssim(1 \vee \gamma) \sum_{\substack{m \in I \\
m \neq 0}} \sum_{(j, k) \in B_{m}}\left|f_{j, k}^{m}\right| \sum_{j^{\prime}>J_{0}} \sup _{m^{\prime}: J_{m^{\prime}}=j^{\prime}}\left\{H_{I}\left(m^{\prime}\right)\right\} 2^{-J_{m} / 2} 2^{j^{\prime} / 2} 2^{-\left(J_{m} \vee j^{\prime}\right) \tilde{s}} \\
& \lesssim(1 \vee \gamma) \sum_{\substack{m \in I \\
m \neq 0}}\left\|F_{m}\right\|_{2} \sum_{j>J_{0}} \sup _{m^{\prime}: J_{m^{\prime}}=j}\left\{H_{I}\left(m^{\prime}\right)\right\} 2^{-J_{m} / 2} 2^{j / 2} 2^{-\left(J_{m} \vee j\right) \tilde{s}} .
\end{aligned}
$$

We decompose summation in the previous display into the sum of the following terms:

$$
\begin{aligned}
T_{1}:= & \sum_{\substack{m \in I \\
m \neq 0}} 2^{-J_{m} / 2}\left\|F_{m}\right\|_{2} \mathbf{1}_{J_{m} \leq j_{n}} \sum_{j=J_{1}}^{J_{m}} \sup _{m^{\prime}: J_{m^{\prime}}=j}\left\{H_{I}\left(m^{\prime}\right)\right\} 2^{j / 2} 2^{-J_{m} \tilde{s}}, \\
T_{2}:= & \sum_{\substack{m \in I \\
m \neq 0}} 2^{-J_{m} / 2}\left\|F_{m}\right\|_{2} \mathbf{1}_{J_{m} \leq j_{n}} \sum_{j=J_{m}+1}^{j_{n}} \sup _{m^{\prime}: J_{m^{\prime}}=j}\left\{H_{I}\left(m^{\prime}\right)\right\} 2^{j / 2} 2^{-j \tilde{s}}, \\
T_{3}:= & \sum_{\substack{m \in I \\
m \neq 0}} 2^{-J_{m} / 2}\left\|F_{m}\right\|_{2} \mathbf{1}_{J_{m} \leq j_{n}} \sum_{j>j_{n}} \sup _{m^{\prime}: J_{m^{\prime}=j}}\left\{H_{I}\left(m^{\prime}\right)\right\} 2^{j / 2} 2^{-j \tilde{s}}, \\
T_{4}:= & \sum_{\substack{m \in I \\
m \neq 0}} 2^{-J_{m} / 2}\left\|F_{m}\right\|_{2} \mathbf{1}_{J_{m}>j_{n}} \sum_{j=J_{1} m^{\prime}: J_{m^{\prime}}=j}^{j_{n}}\left\{H_{I}\left(m^{\prime}\right)\right\} 2^{j / 2} 2^{-J_{m} \tilde{s}}, \\
T_{5}:= & \sum_{\substack{m \in I \\
m \neq 0}} 2^{-J_{m} / 2}\left\|F_{m}\right\|_{2} \mathbf{1}_{J_{m}>j_{n}} \sum_{j=j_{n}+1^{m}: J_{m^{\prime}}=j}^{J_{m}}\left\{H_{I}\left(m^{\prime}\right)\right\} 2^{j / 2} 2^{-J_{m} \tilde{s}}, \\
T_{6}:= & \sum_{\substack{m \in I \\
m \neq 0}} 2^{-J_{m} / 2}\left\|F_{m}\right\|_{2} \mathbf{1}_{J_{m}>j_{n}} \sum_{j>J_{m}} \sup _{m^{\prime}: J_{m^{\prime}}=j}\left\{H_{I}\left(m^{\prime}\right)\right\} 2^{j / 2} 2^{-j \tilde{s}} .
\end{aligned}
$$

We now bound each terms using the definition of $j_{n}$ and of $H_{I}(m)$. Regarding $T_{1}$ when $J_{1} \leq J_{m^{\prime}} \leq$ $J_{m} \leq j_{n}$ we have $H_{I}\left(m^{\prime}\right) \leq \Gamma \xi^{-\mathbf{1}_{0 \in I}} \sqrt{\log (n) / n}$, thus

$$
\begin{aligned}
T_{1} & \lesssim \Gamma \xi^{-\mathbf{1}_{0 \in I}} \sqrt{\frac{\log (n)}{n}} \sum_{m \in I} 2^{-J_{m} \tilde{s}}\left\|F_{m}\right\|_{2} \mathbf{1}_{J_{m} \leq j_{n}} \mathbf{1}_{m \neq 0} \\
& \leq \Gamma \xi^{-\mathbf{1}_{0 \in I}} \sqrt{\frac{\log (n)}{n}}\left\{\sum_{m \in I}\left\|F_{m}\right\|_{2}^{2} \mathbf{1}_{J_{m} \leq j_{n}}\right\}^{1 / 2}\left\{\sum_{m \in I} 2^{-2 J_{m} \tilde{s}_{1}} \mathbf{1}_{J_{m} \leq j_{n}} \mathbf{1}_{m \neq 0}\right\}^{1 / 2} .
\end{aligned}
$$


But, for $m \neq 0$ we have $J_{m}>J_{0}$, and there are no more than $\lesssim 2^{j}$ blocks of wavelets at each level $j$ so in fact

$$
\sum_{m \in I} 2^{-2 J_{m} \tilde{s}} \mathbf{1}_{J_{m} \leq j_{n}} \mathbf{1}_{m \neq 0} \leq \sum_{j=J_{0}+1}^{j_{n}} \sum_{k} 2^{-2 j \tilde{s}} \lesssim \sum_{j=J_{0}+1}^{j_{n}} 2^{j(2 \tilde{s}-1)} \lesssim 2^{-J_{0}(2 \tilde{s}-1)} .
$$

Therefore,

$$
T_{1} \lesssim 2^{-J_{0}(\tilde{s}-1 / 2)} \Gamma \xi^{-\mathbf{1}_{0 \in I}} \sqrt{\frac{\log (n)}{n}}\left\{\sum_{m \in I}\left\|F_{m}\right\|_{2}^{2} \mathbf{1}_{J_{m} \leq j_{n}}\right\}^{1 / 2} .
$$

Since $\tilde{s}>1 / 2$ in this case, $2^{-J_{0}(\tilde{s}-1 / 2)}$ can be made $\ll \delta$ by taking $K_{0}$ in Assumption 1 sufficiently large. The same goes for $T_{2}$ because $\sum_{j=J_{m+1}}^{j_{n}} 2^{j / 2} 2^{-j \tilde{s}} \lesssim 2^{-J_{m}(\tilde{s}-1 / 2)}$, and thus

$$
T_{2} \lesssim 2^{-J_{0}(\tilde{s}-1 / 2)} \Gamma \xi^{-\mathbf{1}_{0 \in I}} \sqrt{\frac{\log (n)}{n}}\left\{\sum_{m \in I}\left\|F_{m}\right\|_{2}^{2} \mathbf{1}_{J_{m} \leq j_{n}}\right\}^{1 / 2}
$$

Regarding $T_{3}$, when $J_{m^{\prime}}>j_{n}$ we have $H_{I}\left(m^{\prime}\right) \leq \gamma 2^{-J_{m^{\prime}}(s+1 / 2)}$, and so

$$
\begin{aligned}
T_{3} & \lesssim \gamma \sum_{m \in I} 2^{-J_{m} / 2}\left\|F_{m}\right\|_{2} \mathbf{1}_{J_{m} \leq j_{n}} \sum_{j>j_{n}} 2^{-j(s+\tilde{s})} \mathbf{1}_{m \neq 0}, \\
& \lesssim \gamma 2^{-j_{n}(s+1 / 2)} 2^{-j_{n}(\tilde{s}-1 / 2)} \sum_{m \in I} 2^{-J_{m} / 2}\left\|F_{m}\right\|_{2} \mathbf{1}_{J_{m} \leq j_{n}} \mathbf{1}_{m \neq 0} \\
& \leq \gamma 2^{-j_{n}(\tilde{s}-1 / 2)} \cdot \sqrt{\frac{\log (n)}{n}} \cdot\left\{\sum_{m \in I}\left\|F_{m}\right\|_{2}^{2} \mathbf{1}_{J_{m} \leq j_{n}}\right\}^{1 / 2}\left\{\sum_{m \in I} 2^{-J_{m}} \mathbf{1}_{J_{m} \leq j_{n}}\right\}^{1 / 2} .
\end{aligned}
$$

But, there are no more than $\lesssim 2^{j}$ wavelets at each level $j$ so in fact

$$
\sum_{m \in I} 2^{-J_{m}} \mathbf{1}_{J_{m} \leq j_{n}} \leq \sum_{j \geq 0}^{j_{n}} \sum_{k} 2^{-j} \lesssim j_{n}
$$

Therefore,

$$
T_{3} \lesssim \gamma \sqrt{\frac{\log (n)}{n}} \cdot \sqrt{j_{n}} 2^{-j_{n}(\tilde{s}-1 / 2)}\left\{\sum_{m \in I}\left\|F_{m}\right\|_{2}^{2} \mathbf{1}_{J_{m} \leq j_{n}}\right\}^{1 / 2}
$$

Regarding $T_{4}$,

$$
\begin{aligned}
T_{4} & \leq \Gamma \xi^{-\mathbf{1}_{0 \in I}} \sqrt{\frac{\log (n)}{n}} \sum_{m \in I} 2^{-J_{m} / 2}\left\|F_{m}\right\|_{2} \mathbf{1}_{J_{m}>j_{n}} \sum_{j=J_{1}}^{j_{n}} 2^{j / 2} 2^{-J_{m} \tilde{s}} \mathbf{1}_{m \neq 0} \\
& \lesssim \Gamma \xi^{-\mathbf{1}_{0 \in I}} \sqrt{\frac{\log (n)}{n}} 2^{j_{n} / 2}\left\{\sum_{m \in I}\left\|F_{m}\right\|_{2}^{2} 1_{J_{m}>j_{n}}\right\}^{1 / 2}\left\{\sum_{m \in I} 2^{-J_{m}(2 \tilde{s}+1)} \mathbf{1}_{J_{m}>j_{n}}\right\}^{1 / 2}
\end{aligned}
$$


But there are no more than $\lesssim 2^{j}$ wavelets at each level $j$ so in fact

$$
\sum_{m \in I} 2^{-J_{m}(2 \tilde{s}+1)} \mathbf{1}_{J_{m}>j_{n}} \leq \sum_{j>j_{n}} \sum_{k} 2^{-j(2 \tilde{s}+1)} \lesssim \sum_{j>j_{n}} 2^{-2 j \tilde{s}} \lesssim 2^{-2 j_{n} \tilde{s}} .
$$

Therefore,

$$
T_{4} \lesssim \Gamma \xi^{-\mathbf{1}_{0 \in I}} \sqrt{\frac{\log (n)}{n}} 2^{-j_{n}(\tilde{s}-1 / 2)}\left\{\sum_{m \in I}\left\|F_{m}\right\|_{2}^{2} \mathbf{1}_{J_{m}>j_{n}}\right\}^{1 / 2}
$$

$\operatorname{Regarding} T_{5}$, for $J_{m^{\prime}}>j_{n}$ we have $H_{I}\left(m^{\prime}\right) \leq \gamma 2^{-J_{m^{\prime}}(s+1 / 2)}$, thus

$$
\begin{aligned}
T_{5} & =\gamma \sum_{m \in I} 2^{-J_{m}(\tilde{s}+1 / 2)}\left\|F_{m}\right\|_{2} \mathbf{1}_{J_{m}>j_{n}} \sum_{j=j_{n}+1}^{J_{m}} 2^{-j s} \mathbf{1}_{m \neq 0} \\
& \lesssim \gamma 2^{-j_{n} s} \sum_{m \in I}\left\|F_{m}\right\|_{2} 2^{-J_{m}(\tilde{s}+1 / 2)} \mathbf{1}_{J_{m}>j_{n}} \\
& \leq \gamma 2^{-j_{n} s}\left\{\sum_{m \in I}\left\|F_{m}\right\|_{2}^{2} \mathbf{1}_{J_{m}>j_{n}}\right\}^{1 / 2}\left\{\sum_{m \in I} 2^{-J_{m}(2 \tilde{s}+1)} \mathbf{1}_{J_{m}>j_{n}}\right\}^{1 / 2} \\
& \lesssim \gamma 2^{-j_{n}(s+\tilde{s})}\left\{\sum_{m \in I}\left\|F_{m}\right\|_{2}^{2} \mathbf{1}_{J_{m}>j_{n}}\right\}^{1 / 2},
\end{aligned}
$$

where the last line follows by equation (S5.14). But by equation (3.2) we have $\gamma 2^{-j_{n}(s+\tilde{s})}=$ $\gamma 2^{-j_{n}(s+1 / 2)} 2^{-j_{n}(\tilde{s}-1 / 2)} \sim \Gamma \sqrt{\log (n) / n} 2^{-j_{n}(\tilde{s}-1 / 2)}$. Therefore,

$$
T_{5} \lesssim 2^{-j_{n}(\tilde{s}-1 / 2)} \Gamma \sqrt{\frac{\log (n)}{n}}\left\{\sum_{m \in I}\left\|F_{m}\right\|_{2}^{2} \mathbf{1}_{J_{m}>j_{n}}\right\}^{1 / 2} .
$$

It remains $T_{6}$ But for $J_{m^{\prime}}>J_{m}>j_{n}$ we again have that $H_{I}\left(m^{\prime}\right) \leq \gamma 2^{-j(s+1 / 2)}$, and thus

$$
\begin{aligned}
T_{6} & =\gamma \sum_{m \in I} 2^{-J_{m} / 2}\left\|F_{m}\right\|_{2} \mathbf{1}_{J_{m}>j_{n}} \sum_{j>J_{m}} 2^{-j(s+\tilde{s})} \\
& \lesssim \gamma \sum_{m \in I}\left\|F_{m}\right\|_{2} 2^{-J_{m}(s+1 / 2+\tilde{s})} \mathbf{1}_{J_{m}>j_{n}} \\
& \leq \gamma\left\{\sum_{m \in I}\left\|F_{m}\right\|_{2}^{2} \mathbf{1}_{J_{m}>j_{n}}\right\}^{1 / 2}\left\{\sum_{m \in I} 2^{-J_{m}(2 s+1+2 \tilde{s})} \mathbf{1}_{J_{m}>j_{n}}\right\}^{1 / 2} \\
& \lesssim \gamma 2^{-j_{n}(s+\tilde{s})}\left\{\sum_{m \in I}\left\|F_{m}\right\|_{2}^{2} \mathbf{1}_{J_{m}>j_{n}}\right\}^{1 / 2},
\end{aligned}
$$

where the last line follows by the same arguments that led to equation (S5.14). As before,

$$
T_{6} \lesssim 2^{-j_{n}(\tilde{s}-1 / 2)} \Gamma \sqrt{\frac{\log (n)}{n}} \cdot\left\{\sum_{m \in I}\left\|F_{m}\right\|_{2}^{2} \mathbf{1}_{J_{m}>j_{n}}\right\}^{1 / 2} .
$$


The bound for $R_{3}$ is obtained by equation (S5.7) and all the estimates on $T_{1}, \ldots, T_{6}$, and by taking the constants in Assumption 1 sufficiently large.

Bound on $R_{3}$ : case $0<s \leq 1 / 2$. We note that the equation (S5.7) remains valid in this case, as well as the decomposition of equations (S5.8) to (S5.13). We again bound each of these terms. Regarding $T_{1}$ when $J_{1} \leq J_{m^{\prime}} \leq J_{m} \leq j_{n}$, we have $H_{I}\left(m^{\prime}\right) \leq \Gamma \xi^{-\mathbf{1}_{0 \in I}} 2^{-J_{m^{\prime}} / 2} \varepsilon_{n}^{*}(s)$, thus

$$
\begin{aligned}
T_{1} & \leq \Gamma \xi^{-\mathbf{1}_{0 \in I}} \sum_{m \in I} 2^{-J_{m} / 2}\left\|F_{m}\right\|_{2} \mathbf{1}_{J_{m} \leq j_{n}} \sum_{j=J_{1}}^{J_{m}} \varepsilon_{n}^{*}(s) 2^{-J_{m} s} \mathbf{1}_{m \neq 0} \\
& \lesssim \Gamma \xi^{-\mathbf{1}_{0 \in I}} \varepsilon_{n}^{*}(s) \sum_{m \in I} J_{m} 2^{-J_{m}(s+1 / 2)}\left\|F_{m}\right\|_{2} \mathbf{1}_{J_{m} \leq j_{n}} \mathbf{1}_{m \neq 0} \\
& \leq \Gamma \xi^{-\mathbf{1}_{0 \in I}} \varepsilon_{n}^{*}(s)\left\{\sum_{m \in I}\left\|F_{m}\right\|_{2}^{2} \mathbf{1}_{J_{m} \leq j_{n}} \mathbf{1}_{m \neq 0}\right\}^{1 / 2}\left\{\sum_{m \in I} J_{m} 2^{-J_{m}(2 s+1)} \mathbf{1}_{J_{m} \leq j_{n}} \mathbf{1}_{m \neq 0}\right\}^{1 / 2} \\
& \leq \Gamma \xi^{-\mathbf{1}_{0 \in I}} J_{0} 2^{-J_{0} s} \varepsilon_{n}^{*}(s)\left\{\sum_{m \in I}\left\|F_{m}\right\|_{2}^{2} \mathbf{1}_{J_{m} \leq j_{n}} \mathbf{1}_{m \neq 0}\right\}^{1 / 2}\left\{\sum_{m \in I} 2^{-J_{m}} \mathbf{1}_{J_{m} \leq j_{n}} \mathbf{1}_{m \neq 0}\right\}^{1 / 2},
\end{aligned}
$$

where the last line follows for $J_{0}$ taken sufficiently large, because $m \neq 0 \Rightarrow J_{m}>J_{0}$, and thus $J_{m} 2^{-2 J_{m} s} \leq J_{0} 2^{-2 J_{0} s}$. Regarding $T_{2}$, we have using the same arguments

$$
\begin{aligned}
T_{2} & \leq \Gamma \xi^{-\mathbf{1}_{0 \in I}} \varepsilon_{n}^{*}(s) \sum_{m \in I} 2^{-J_{m} / 2}\left\|F_{m}\right\|_{2} \mathbf{1}_{J_{m} \leq j_{n}} \sum_{j=J_{m}+1}^{j_{n}} 2^{-j s} \mathbf{1}_{m \neq 0} \\
& \lesssim \Gamma \xi^{-\mathbf{1}_{0 \in I}} \varepsilon_{n}^{*}(s) \sum_{m \in I} 2^{-J_{m}(s+1 / 2)}\left\|F_{m}\right\|_{2} \mathbf{1}_{J_{m} \leq j_{n}} \mathbf{1}_{m \neq 0} \\
& \leq \Gamma \xi^{-\mathbf{1}_{0 \in I}} \varepsilon_{n}^{*}(s)\left\{\sum_{m \in I}\left\|F_{m}\right\|_{2}^{2} \mathbf{1}_{J_{m} \leq j_{n}} \mathbf{1}_{m \neq 0}\right\}^{1 / 2}\left\{\sum_{m \in I} 2^{-J_{m}(2 s+1)} \mathbf{1}_{J_{m} \leq j_{n}} \mathbf{1}_{m \neq 0}\right\}^{1 / 2} \\
& \leq \Gamma \xi^{-\mathbf{1}_{0 \in I}} 2^{-J_{0} s} \varepsilon_{n}^{*}(s)\left\{\sum_{m \in I}\left\|F_{m}\right\|_{2}^{2} \mathbf{1}_{J_{m} \leq j_{n}} \mathbf{1}_{m \neq 0}\right\}^{1 / 2}\left\{\sum_{m \in I} 2^{-J_{m}} \mathbf{1}_{J_{m} \leq j_{n}} \mathbf{1}_{m \neq 0}\right\}^{1 / 2} .
\end{aligned}
$$

Now for $T_{3}$,

$$
\begin{aligned}
T_{3} & \leq \gamma \sum_{m \in I} 2^{-J_{m} / 2}\left\|F_{m}\right\|_{2} \mathbf{1}_{J_{m} \leq j_{n}} \sum_{j>j_{n}} 2^{-2 j s} \mathbf{1}_{m \neq 0} \\
& \lesssim \gamma 2^{-2 j_{n} s} \sum_{m \in I} 2^{-J_{m} / 2}\left\|F_{m}\right\|_{2} \mathbf{1}_{J_{m} \leq j_{n}} \mathbf{1}_{m \neq 0} \\
& \leq \gamma 2^{-2 j_{n} s}\left\{\sum_{m \in I}\left\|F_{m}\right\|_{2}^{2} 1_{J_{m} \leq j_{n}} \mathbf{1}_{m \neq 0}\right\}^{1 / 2}\left\{\sum_{m \in I} 2^{-J_{m}} \mathbf{1}_{J_{m} \leq j_{n}} \mathbf{1}_{m \neq 0}\right\}^{1 / 2} .
\end{aligned}
$$

But we note that $\gamma 2^{-2 j_{n} s} \lesssim \Gamma^{2} \varepsilon_{n}^{*}(s)^{2} / \gamma$ by equation (S5.3), and hence we have,

$$
T_{3} \lesssim \frac{\Gamma \varepsilon_{n}^{*}(s)}{\gamma} \cdot \Gamma \varepsilon_{n}^{*}(s)\left\{\sum_{m \in I}\left\|F_{m}\right\|_{2}^{2} \mathbf{1}_{J_{m} \leq j_{n}} \mathbf{1}_{m \neq 0}\right\}^{1 / 2}\left\{\sum_{m \in I} 2^{-J_{m}} \mathbf{1}_{J_{m} \leq j_{n}} \mathbf{1}_{m \neq 0}\right\}^{1 / 2} .
$$


Now for $T_{4}$, we have

$$
\begin{aligned}
T_{4} & \leq \Gamma \xi^{-\mathbf{1}_{0 \in I}} \varepsilon_{n}^{*}(s) \sum_{m \in I} 2^{-J_{m}(s+1 / 2)}\left\|F_{m}\right\|_{2} \mathbf{1}_{J_{m}>j_{n}}\left(j_{n}-J_{1}\right) \mathbf{1}_{m \neq 0} \\
& \leq j_{n} \Gamma \xi^{-\mathbf{1}_{0 \in I}} \varepsilon_{n}^{*}(s)\left\{\sum_{m \in I}\left\|F_{m}\right\|_{2}^{2} \mathbf{1}_{J_{m}>j_{n}}\right\}^{1 / 2}\left\{\sum_{m \in I} 2^{-J_{m}(2 s+1)} \mathbf{1}_{J_{m}>j_{n}}\right\}^{1 / 2} .
\end{aligned}
$$

Similarly,

$$
\begin{aligned}
T_{5} & \leq \gamma \sum_{m \in I} 2^{-J_{m}(s+1 / 2)}\left\|F_{m}\right\|_{2} \mathbf{1}_{J_{m}>j_{n}} \sum_{j=j_{n}+1}^{J_{m}} 2^{-j s} \mathbf{1}_{m \neq 0}, \\
& \lesssim \gamma 2^{-j_{n} s} \sum_{m \in I} 2^{-J_{m}(s+1 / 2)}\left\|F_{m}\right\|_{2} \mathbf{1}_{J_{m}>j_{n}} \\
& \lesssim \gamma 2^{-j_{n} s}\left\{\sum_{m \in I}\left\|F_{m}\right\|_{2}^{2} \mathbf{1}_{J_{m}>j_{n}}\right\}^{1 / 2}\left\{\sum_{m \in I} 2^{-J_{m}(2 s+1)} \mathbf{1}_{J_{m}>j_{n}}\right\}^{1 / 2} .
\end{aligned}
$$

Finally,

$$
\begin{aligned}
T_{6} & \leq \gamma \sum_{m \in I} 2^{-J_{m} / 2}\left\|F_{m}\right\|_{2} \mathbf{1}_{J_{m}>j_{n}} \sum_{j>J_{m}} 2^{-2 j s} \\
& \lesssim \gamma \sum_{m \in I} 2^{-J_{m}(2 s+1 / 2)}\left\|F_{m}\right\|_{2} \mathbf{1}_{J_{m}>j_{n}} \\
& \lesssim \gamma\left\{\sum_{m \in I}\left\|F_{m}\right\|_{2}^{2} \mathbf{1}_{J_{m}>j_{n}}\right\}^{1 / 2}\left\{\sum_{m \in I} 2^{-J_{m}(4 s+1)} \mathbf{1}_{J_{m}>j_{n}}\right\}^{1 / 2} \\
& \leq \gamma 2^{-j_{n} s}\left\{\sum_{m \in I}\left\|F_{m}\right\|_{2}^{2} \mathbf{1}_{J_{m}>j_{n}}\right\}^{1 / 2}\left\{\sum_{m \in I} 2^{-J_{m}(2 s+1)} \mathbf{1}_{J_{m}>j_{n}}\right\}^{1 / 2} .
\end{aligned}
$$

The bound for $R_{3}$ is obtained by equation (S5.7) and all the estimates on $T_{1}, \ldots, T_{6}$ and by taking the constants in Assumption 1 sufficiently large.

Proof of Proposition S5.1. We use that $F_{m}=\sum_{(j, k) \in B_{m}}\left\langle F_{m}, \varphi_{j, k}\right\rangle\left(\varphi_{j, k}-\mathbb{E}_{L}\left[\varphi_{j, k}\right]\right)$ by Proposition 7.1-(1). Then, by Proposition 7.1-(5) we deduce that,

$$
\begin{aligned}
\left|S_{\overline{\boldsymbol{F}}_{I}}(x)\right| & \leq \sum_{m \in I^{c}} \sum_{(j, k) \in B_{m}}\left|\left\langle F_{m}, \varphi_{j, k}\right\rangle\right|\left|\varphi_{j, k}(x)-\mathbb{E}_{L}\left[\varphi_{j, k}\right]\right| \\
& \leq \sum_{j \geq 0} \sup _{m \in I^{c}, J_{m}=j}\left\{\left\|F_{m}\right\|_{2} \sum_{k}\left|\varphi_{j, k}(x)-\mathbb{E}_{L}\left[\varphi_{j, k}\right]\right|\right\} \\
& \lesssim \sum_{j \geq 0} \sup _{m: J_{m}=j}\left\{H_{I}(m) 2^{J_{m} / 2}\right\}
\end{aligned}
$$

By the same argument as in the proof of Corollary 3.1 , the previous is seen to be $\lesssim \max \left\{(\Gamma / \gamma)^{\frac{2 s}{2 s+1}}, \Gamma(\gamma / \Gamma)^{\frac{1}{2 s+1}}\right\} \varepsilon_{n}^{*}(s)$ when $s>1 / 2$, and $\lesssim \log \left(\Gamma \varepsilon_{n}^{*}(s) / \gamma\right) \Gamma \varepsilon_{n}^{*}(s)$ otherwise. 
Proof of Proposition S5.2, Item (1). We define the function $h(x):=p_{L}(x) A\left(S_{\overline{\boldsymbol{F}}_{I}}(x)\right)$. With this definition, we can rewrite

$$
\begin{aligned}
\Sigma_{j, k, j^{\prime}, k^{\prime}}^{\overline{\boldsymbol{F}}_{I}}= & \int h \cdot\left(\varphi_{j, k}-\mathbb{E}_{L}\left[\varphi_{j, k}\right]\right)\left(\varphi_{j^{\prime}, k^{\prime}}-\mathbb{E}_{L}\left[\varphi_{j^{\prime}, k^{\prime}}\right]\right) \\
= & \int h \cdot \varphi_{j, k} \varphi_{j^{\prime}, k^{\prime}}-\mathbb{E}_{L}\left[\varphi_{j, k}\right] \int h \cdot \varphi_{j^{\prime}, k^{\prime}} \\
& -\mathbb{E}_{L}\left[\varphi_{j^{\prime}, k^{\prime}}\right] \int h \cdot \varphi_{j, k}+\mathbb{E}_{L}\left[\varphi_{j, k}\right] \mathbb{E}_{L}\left[\varphi_{j^{\prime}, k^{\prime}}\right] \int h .
\end{aligned}
$$

By assumption $\left\|S_{\overline{\boldsymbol{F}}_{I}}\right\|_{\infty} \leq 1$, thus $\left\|A\left(S_{\overline{\boldsymbol{F}}_{I}}(\cdot)\right)\right\|_{\infty} \lesssim 1$ too. Further, for any $g \in B_{\infty, \infty}^{s}$ we have $\left|\int g \varphi_{j, k}\right| \leq C\|g\|_{\infty, \infty, s} 2^{-j(s+1 / 2)}$ for a universal constant depending eventually on $s$; see for instance Giné and Nickl (2016). Thus, we deduce that for $C>0$ eventually depending on $\tilde{s}$,

$$
\left|\Sigma_{j, k, j^{\prime}, k^{\prime}}^{\overline{\boldsymbol{F}}_{I}}\right| \leq\left|\int h \cdot \varphi_{j, k} \varphi_{j^{\prime}, k^{\prime}}\right|+C \max \left\{\|p\|_{\infty, \infty, \tilde{s}},\|h\|_{\infty, \infty, \tilde{s}}\right\} 2^{-j(\tilde{s}+1 / 2)} 2^{-j^{\prime}(\tilde{s}+1 / 2)} .
$$

We remark that $p=\exp \{L\}$, and thus $\|p\|_{\infty, \infty, \tilde{s}}$ is in turn bounded by a constant depending only on $(R, s)$. Further $(j, k) \neq\left(j^{\prime}, k^{\prime}\right)$, thus $\int \varphi_{j, k} \varphi_{j^{\prime}, k^{\prime}}=0$, hence for any $y \in[0,1]$,

$$
\int h \cdot \varphi_{j, k} \varphi_{j^{\prime}, k^{\prime}}=\int_{[0,1]}(h(x)-h(y)) \varphi_{j, k}(x) \varphi_{j^{\prime}, k^{\prime}}(x) \mathrm{d} x .
$$

If $S_{j, k, j^{\prime}, k^{\prime}}:=\operatorname{supp} \varphi_{j, k} \cap \operatorname{supp} \varphi_{j^{\prime}, k^{\prime}}=\varnothing$, then $\int h \cdot \varphi_{j, k} \varphi_{j^{\prime}, k^{\prime}}=0$ and the result is immediate. We now consider that $S_{j, k, j^{\prime}, k^{\prime}} \neq \varnothing$. In this case, pick $y \in S_{j, k, j^{\prime}, k^{\prime}}$ arbitrary and remark that for all $x \in S_{j, k, j^{\prime}, k^{\prime}}$ we have $|x-y| \leq C 2^{-j \vee j^{\prime}}$ for a constant $C>0$ depending only on the wavelet basis. Then by equation (S5.16),

$$
\begin{aligned}
\left|\int h \cdot \varphi_{j, k} \varphi_{j^{\prime}, k^{\prime}}\right| & \leq \sup _{x \neq y} \frac{|h(x)-h(y)|}{|x-y|^{\tilde{s}}} \int_{[0,1]}|x-y|^{\tilde{s}}\left|\varphi_{j, k}(x) \varphi_{j^{\prime}, k^{\prime}}(x)\right| \mathrm{d} x \\
& \leq \sup _{x \neq y} \frac{|h(x)-h(y)|}{|x-y|^{\tilde{s}}} \cdot 2^{-\left(j \vee j^{\prime}\right) \tilde{s}} \int\left|\varphi_{j, k} \varphi_{j^{\prime}, k^{\prime}}\right| .
\end{aligned}
$$

Then, we obtain the result of the proposition by bounding the $\tilde{s}$-Hölder norm of $h$. For all $x, y \in[0,1]$,

$$
\begin{aligned}
|h(x)-h(y)| & \leq\left|p_{L}(x)-p_{L}(y)\right| \cdot\left|A\left(S_{\overline{\boldsymbol{F}}_{I}}(x)\right)\right|+p_{L}(y)\left|A\left(S_{\overline{\boldsymbol{F}}_{I}}(x)\right)-A\left(S_{\overline{\boldsymbol{F}}_{I}}(y)\right)\right| \\
& =\left|e^{L(x)}-e^{L(y)}\right| \cdot\left|A\left(S_{\overline{\boldsymbol{F}}_{I}}(x)\right)\right|+e^{L(y)}\left|A\left(S_{\overline{\boldsymbol{F}}_{I}}(x)\right)-A\left(S_{\overline{\boldsymbol{F}}_{I}}(y)\right)\right| .
\end{aligned}
$$

Whenever $L \in \Sigma(R, s)$, there is a constant $C>0$ depending only on $R$ such that $\left|e^{L(x)}-e^{L(y)}\right| \leq$ $C|L(x)-L(y)|$ and $\sup _{x} e^{L(x)} \leq C$. Also $\left\|S_{\overline{\boldsymbol{F}}_{I}}\right\|_{\infty} \leq 1$ thus $\sup _{x}\left|A\left(S_{\overline{\boldsymbol{F}}_{I}}(x)\right)\right| \lesssim 1$ and $\sup _{x}\left|A^{\prime}\left(S_{\overline{\boldsymbol{F}}_{I}}(x)\right)\right| \lesssim$ 1. We deduce,

$$
|h(x)-h(y)| \lesssim C|L(x)-L(y)|+C\left|S_{\overline{\boldsymbol{F}}_{I}}(x)-S_{\overline{\boldsymbol{F}}_{I}}(y)\right| .
$$

By construction $0<\tilde{s}<1$, which implies that the $\tilde{s}$-Hölder norm is equivalent to the $\|\cdot\|_{\infty, \infty, \tilde{s}}$ norm (Giné and Nickl, 2016, Equations 4.149 and 4.152). Then, since $L \in \Sigma(R, s) \subseteq \Sigma(R, \tilde{s})$,

$$
\sup _{x \neq y} \frac{|h(x)-h(y)|}{|x-y|^{\tilde{s}}} \lesssim R C+C\left\|S_{\overline{\boldsymbol{F}}_{I}}\right\|_{\infty, \infty, \tilde{s}}
$$


By equivalence of norms, $\|h\|_{\infty, \infty, \tilde{s}}$ is also bounded by a constant (eventually depending on $\tilde{s}$ ) times the last display. Hence, the conclusion follows by combining equations (S5.15) and (S5.17) with the last display.

Proof of Proposition S5.2, Item (2). By the Item (1),

$$
\begin{aligned}
\sum_{k}\left|\Sigma_{j, k, j^{\prime}, k^{\prime}}\right| & \lesssim\left(1+\left\|S_{\overline{\boldsymbol{F}}_{I}}\right\|_{\infty, \infty, \tilde{s})}\left\{2^{-j^{\prime} \tilde{s}} \int\left|\varphi_{j^{\prime}, k^{\prime}}\right| \sum_{k}\left|\varphi_{j, k}\right|+2^{-j^{\prime}(\tilde{s}+1 / 2)} \sum_{k} 2^{-j(\tilde{s}+1 / 2)}\right\}\right. \\
& \lesssim\left(1+\left\|S_{\overline{\boldsymbol{F}}_{I}}\right\|_{\infty, \infty, \tilde{s})}\left\{2^{-j^{\prime} \tilde{s}} 2^{j / 2} \int\left|\varphi_{j^{\prime}, k^{\prime}}\right|+2^{-j^{\prime}(\tilde{s}+1 / 2)} 2^{-j \tilde{s}} 2^{j / 2}\right\}\right.
\end{aligned}
$$

where the last line follows by Proposition 7.1-(5) and also because there are no more than $\lesssim 2^{j}$ wavelets at each level $j$. The conclusion follows because $\left\|\varphi_{j^{\prime}, k^{\prime}}\right\|_{1} \lesssim 2^{-j^{\prime}}$ for all $\left(j^{\prime}, k^{\prime}\right) \in \mathcal{V}$.

Proof of Proposition S5.2, Item (3). Note that if $(j, k) \in B_{0}$ then

$$
\begin{aligned}
2^{j(\tilde{s}+1 / 2)}\left|\left\langle S_{\overline{\boldsymbol{F}}_{I}}, \varphi_{j, k}\right\rangle\right| & \leq 2^{j(\tilde{s}+1 / 2)}\left\|S_{\overline{\boldsymbol{F}}_{I}}\right\|_{\infty}\left\|\varphi_{j, k}\right\|_{1} \\
& \leq 2^{J_{0} \tilde{s}}\left\|S_{\overline{\boldsymbol{F}}_{I}}\right\|_{\infty} \\
& \lesssim 2^{J_{0} \tilde{s}} \log \left(\Gamma \varepsilon_{n}^{*}(s) / \gamma\right) \Gamma \varepsilon_{n}^{*}(s),
\end{aligned}
$$

where the last line follows by Proposition S5.1. In the case where $(j, k) \in B_{0}^{c} \cap\left\{(j, k): j \leq j_{n}\right\}$, then by Proposition 7.1-(2) (since under Assumption 1 we can assume wlog that $J_{0}$ is arbitrarily large),

$$
\begin{aligned}
2^{j(\tilde{s}+1 / 2)}\left|\left\langle S_{\overline{\boldsymbol{F}}_{I}}, \varphi_{j, k}\right\rangle\right| & \leq 2^{j(\tilde{s}+1 / 2)} \sup _{m \in I^{c}, m \neq 0}\left|\left\langle F_{m}, \varphi_{j, k}\right\rangle\right| \\
& \leq 2^{j(\tilde{s}+1 / 2)} \Gamma \xi^{-\mathbf{1}_{0 \in I}} \sup _{m: J_{m}=j}\left\{\rho_{m}\right\} \\
& \leq \xi^{-\mathbf{1}_{0 \in I}} \begin{cases}2^{j_{n}(s+1 / 2)} \Gamma \sqrt{\log (n) / n} & \text { if } s>1 / 2, \\
\Gamma 2^{j_{n} s} \varepsilon_{n}^{*}(s) & \text { if } s>1 / 2,\end{cases}
\end{aligned}
$$

where the last line follows from the definition of $\rho_{m}$ and because $j \leq j_{n}$. Then by definition of $j_{n}$ in equation (3.2), for all $(j, k) \in B_{0}^{c} \cap\left\{(j, k): j \leq j_{n}\right\}$

$$
2^{j(\tilde{s}+1 / 2)}\left|\left\langle S_{\overline{\boldsymbol{F}}_{I}}, \varphi_{j, k}\right\rangle\right| \leq \xi^{-\mathbf{1}_{0 \in I}} \gamma .
$$

Finally, in the case where $(j, k) \in B_{0}^{c} \cap\left\{(j, k): j>j_{n}\right\}$, then by Proposition 7.1-(2)

$$
2^{j(\tilde{s}+1 / 2)}\left|\left\langle S_{\overline{\boldsymbol{F}}_{I}}, \varphi_{j, k}\right\rangle\right| \leq 2^{j(\tilde{s}+1 / 2)} \sup _{m \in I^{c}, m \neq 0}\left|\left\langle F_{m}, \varphi_{j, k}\right\rangle\right| \leq \gamma .
$$

Combining equations (S5.18) to (S5.20), there is a universal $C>0$ such that,

$$
\left\|S_{\overline{\boldsymbol{F}}_{I}}\right\|_{\infty, \infty, \tilde{s}} \leq \max \left\{2^{J_{0} \tilde{s}} \log \left(\Gamma \varepsilon_{n}^{*}(s) / \gamma\right) \Gamma \varepsilon_{n}^{*}(s), \gamma\right\} .
$$

So if $K_{1}$ in Assumption 1 is large enough we have that $\left\|S_{\overline{\boldsymbol{F}}_{I}}\right\|_{\infty, \infty, \tilde{s}} \leq \gamma$. 


\section{S5.3. Proofs of Lemmas 5.1 and 5.2}

Proof of Lemma 5.1. We prove the lemma by computing an upper bound on $\Pi\left(\mathcal{A}_{I}\right)$ and a lower bound on $\Pi\left(\tilde{\mathcal{A}}_{I}\right)$. We start with the upper bound. Recall that by construction we have $F_{m}^{\boldsymbol{\theta}}=$ $\sum_{(j, k) \in B_{m}}\left(\theta_{j, k}-\theta_{j, k}^{L}\right)\left(\varphi_{j, k}-\mathbb{E}_{L}\left[\varphi_{j, k}\right]\right)$. Take $m \in I \cap\left\{m: J_{m}>j_{n}\right\}$, assuming without loss of generality that this set is not empty. Since $j_{n} \gg 1$ we have by Proposition 7.1

$$
\sup _{(j, k) \in B_{m}}\left|\theta_{j, k}-\theta_{j, k}^{L}\right|=\sup _{(j, k) \in B_{m}}\left|\left\langle F_{m}^{\boldsymbol{\theta}}, \varphi_{j, k}\right\rangle\right|=\left\|F_{m}^{\boldsymbol{\theta}}\right\|_{2} .
$$

Hence, if $\boldsymbol{F}_{I}^{\boldsymbol{\theta}} \in \mathcal{A}_{I}$, then

$$
\sup _{(j, k) \in B_{m}}\left|\theta_{j, k}-\theta_{j, k}^{L}\right| \geq \gamma 2^{-J_{m}(s+1 / 2)} .
$$

But by assumption $\left|\theta_{j, k}^{L}\right| \leq R 2^{-j(s+1 / 2)}$. This implies that if $K_{3}>R$ in Assumption 1 then $\gamma>R$ and it must the case that $\boldsymbol{F}_{I}^{\boldsymbol{\theta}} \in \mathcal{A}_{I}$ implies that $\theta_{\psi(m)} \neq 0$ for $m \in I \cap\left\{m: J_{m}>j_{n}\right\}$. We deduce that

$$
\Pi\left(\mathcal{A}_{I}\right) \leq \prod_{\substack{m \in I \\ J_{m}>j_{n}}} \omega_{J_{m}} \leq \exp \left\{\left(1+\mu_{\star}\right) \log (2) \sum_{m \in I} J_{m} \mathbf{1}_{J_{m}>j_{n}}\right\} .
$$

The previous bound is true for any $I \subseteq \mathbb{Z}_{+}$. We remark, however, that $\omega_{J_{m}}=0$ when $J_{m}>$ $\log (n) / \log (2)$, whence the claim that $\Pi\left(\mathcal{A}_{I}\right) / \Pi\left(\tilde{\mathcal{A}}_{I}\right)=0$ if $I \cap\left\{m: J_{m}>\log (n) / \log (2)\right\} \neq \varnothing$ (it is always the case that $\Pi\left(\tilde{\mathcal{A}}_{I}\right)>0$, see below). We now compute a lower bound on $\Pi\left(\tilde{\mathcal{A}}_{I}\right)$. Consider the set

$$
E:=\left\{\boldsymbol{F}_{I}^{\boldsymbol{\theta}}: \begin{array}{c}
m \in I \cap\left\{m: J_{m} \leq j_{n}\right\} \Longrightarrow \sup _{(j, k) \in B_{m}} \sqrt{\left|B_{m}\right|}\left|\theta_{j, k}-\theta_{j, k}^{L}\right| \leq \eta_{n}, \\
m \in I \cap\left\{m: J_{m}>j_{n}\right\} \Longrightarrow \theta_{\psi(m)}=0 .
\end{array}\right\} .
$$

We will show that $E \subseteq \tilde{\mathcal{A}}_{I}$ for suitable choice of $\eta_{n}$, and then we will bound $\Pi\left(\tilde{\mathcal{A}}_{I}\right) \geq \Pi(E)$. Pick $\boldsymbol{F}_{I}^{\boldsymbol{\theta}} \in E$. By Proposition 7.1, we deduce that

$$
\begin{aligned}
\mathbb{E}_{L}\left[S_{\boldsymbol{F}_{I}}^{2}\right] & \lesssim \sum_{m \in I}\left\|F_{m}\right\|_{2}^{2} \\
& \lesssim \eta_{n}^{2} \sum_{m \in I} \mathbf{1}_{J_{m} \leq j_{n}}+\sum_{m \in I} \sup _{(j, k) \in B_{m}}\left|\theta_{j, k}^{L}\right|^{2} \mathbf{1}_{J_{m}>j_{n}} \\
& \leq \eta_{n}^{2} \sum_{m \in I} \mathbf{1}_{J_{m} \leq j_{n}}+R^{2} \sum_{m \in I} 2^{-J_{m}(2 s+1)} \mathbf{1}_{J_{m}>j_{n}} .
\end{aligned}
$$

We deduce from Proposition 7.2 that if $K_{3}$ in the Assumption 1 is sufficiently large and if $\eta_{n}=$ $a \delta \sqrt{\log (n) / n}$ for small enough constant $a>0$, then $\mathbb{E}_{L}\left[S_{\boldsymbol{F}_{I}}^{2}\right] \leq \delta^{2} \mathcal{E}_{I}^{2}$ for all $\boldsymbol{F}_{I}^{\boldsymbol{\theta}} \in E$. Similarly, by Proposition 7.1-(5),

$$
\begin{aligned}
\left\|S_{\boldsymbol{F}_{I}}\right\|_{\infty} & =\sup _{x \in[0,1]}\left|\sum_{m \in I} \sum_{(j, k) \in B_{m}}\left(\theta_{j, k}-\theta_{j, k}^{L}\right)\left(\varphi_{j, k}(x)-\mathbb{E}_{L}\left[\varphi_{j, k}\right]\right)\right| \\
& \leq \sum_{j \geq J_{0}} \sup _{\substack{m_{m} \in I \\
J_{m}=j}} \sup _{\left(j^{\prime}, k^{\prime}\right) \in B_{m}}\left|\theta_{j^{\prime}, k^{\prime}}-\theta_{j^{\prime}, k^{\prime}}^{L}\right| \sup _{x \in[0,1]} \sum_{k}\left|\varphi_{j, k}(x)-\mathbb{E}_{L}\left[\varphi_{j, k}\right]\right|
\end{aligned}
$$




$$
\begin{aligned}
& \lesssim \eta_{n} \sum_{j=0}^{j_{n}} 2^{j / 2}+\sum_{j>j_{n}} 2^{j / 2} \cdot R 2^{-j(s+1 / 2)} \\
& \lesssim \eta_{n} 2^{j_{n} / 2}+R 2^{-j_{n} s} .
\end{aligned}
$$

Thus, if $\eta_{n}$ is taken as above and $K_{1}$ in Assumption 1 is large enough, it is the case that $\left\|S_{\boldsymbol{F}_{I}}\right\|_{\infty} \leq \delta$ for all $\boldsymbol{F}_{I}^{\boldsymbol{\theta}} \in E$. We thus have proven that $E \subseteq \tilde{\mathcal{A}}_{I}$. Further,

$$
\Pi(E) \geq \prod_{\substack{m \in I \\ J_{m} \leq j_{n}}} \prod_{(j, k) \in B_{m}}\left(\omega_{j} Q_{j}\left(\sqrt{\left|B_{m}\right|}\left|\theta_{j, k}-\theta_{j, k}^{L}\right| \leq \eta_{n}\right)\right) \prod_{\substack{m \in I \\ J_{m}>j_{n}}} \prod_{(j, k) \in B_{m}}\left(1-\omega_{j}\right)
$$

First we note that

$$
\begin{aligned}
\prod_{\substack{m \in I \\
J_{m}>j_{n}}} \prod_{(j, k) \in B_{m}}\left(1-\omega_{j}\right) & \geq \exp \left\{-2 \sum_{m \in I} \sum_{(j, k) \in B_{m}} 2^{-j\left(1+\mu_{\star}\right)} \mathbf{1}_{J_{m} \leq j_{n}}\right\} \\
& \geq \exp \left\{-2 \sum_{j=0}^{j_{n}} \sum_{k} 2^{-j\left(1+\mu_{\star}\right)}\right\} \\
& \geq C_{1},
\end{aligned}
$$

for a universal constant $C_{1}>0$, where the third line follows because there are no more than $\lesssim 2^{j}$

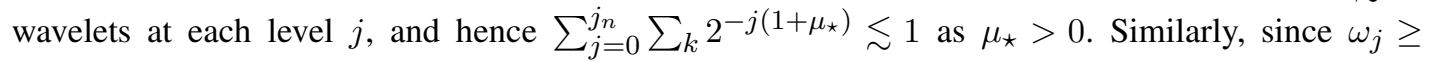
$a_{1} 2^{-j\left(1+b_{1}\right)}$,

$$
\begin{aligned}
\prod_{\substack{m \in I \\
J_{m} \leq j_{n}}} \prod_{(j, k) \in B_{m}} \omega_{j} & \geq \exp \left\{-\log \frac{1}{a_{1}} \sum_{m \in I}\left|B_{m}\right| \mathbf{1}_{J_{m} \leq j_{n}}-b_{1} \sum_{m \in I} \sum_{(j, k) \in B_{m}} j \mathbf{1}_{J_{m}>j_{n}}\right\} \\
& \geq \exp \left\{-\log \frac{1}{a_{1}} \sum_{m \in I}\left|B_{m}\right| \mathbf{1}_{J_{m} \leq j_{n}}-b_{1} \sum_{m \in I}\left|B_{m}\right| J_{m} \mathbf{1}_{J_{m}>j_{n}}\right\}
\end{aligned}
$$

because by construction $(j, k) \in B_{m} \Longrightarrow j \leq J_{m}$ (with equality whenever $m \geq 1$ ). We remark that $\left|B_{0}\right| J_{0} \lesssim J_{0} 2^{J_{0}} \leq \log (n)$ (by choosing $K_{1}$ large enough in Assumption 1), and $\left|B_{m}\right| J_{m}=J_{m} \leq$ $j_{n} \lesssim \log (n)$ for all $1 \leq m \leq j_{n}$. Hence, there is a universal constant $C_{2}>0$ such that

$$
\prod_{\substack{m \in I \\ J_{m} \leq j_{n}}} \prod_{(j, k) \in B_{m}} \omega_{j} \geq \exp \left\{-C_{2} \log (n) \sum_{m \in I} \mathbf{1}_{J_{m} \leq j_{n}}\right\}
$$

Finally, for a universal constant $C_{3}>0$,

$$
\begin{aligned}
Q_{j}\left(\sqrt{\left|B_{m}\right|}\left|\theta_{j, k}-\theta_{j, k}^{L}\right| \leq \eta_{n}\right) & =F\left(\left|X-2^{j\left(s_{0}+1 / 2\right)} \theta_{j, k}^{L}\right| \leq 2^{j\left(s_{0}+1 / 2\right)} \sqrt{\frac{\eta_{n}}{\left|B_{m}\right|}}\right) \\
& \geq C_{3} 2^{j\left(s_{0}+1 / 2\right)} \sqrt{\frac{\eta_{n}}{\left|B_{m}\right|}} \\
& \geq n^{-1 / 2} .
\end{aligned}
$$


The second inequality is true because by assumption $2^{j\left(s_{0}+1 / 2\right)}\left|\theta_{j, k}^{L}\right| \leq R 2^{j\left(s_{0}-s\right)} \leq R$ since $s \geq$ $s_{0}$; henceforth the distribution $F$ has density bounded from below by $C_{3} / 2$ in a neighborhood of $2^{j\left(s_{0}+1 / 2\right)}\left|\theta_{j, k}^{L}\right|$, with $C_{3}$ not depending on the choice of the wavelet coefficient. The last inequality is true whenever $K_{1}$ in Assumption 1 is taken large enough. Then,

$$
\prod_{\substack{m \in I \\ J_{m} \leq j_{n}}} \prod_{(j, k) \in B_{m}} Q_{j}\left(\sqrt{\left|B_{m}\right|}\left|\theta_{j, k}-\theta_{j, k}^{L}\right| \leq \eta_{n}\right) \geq \exp \left\{-\frac{\log (n)}{2} \sum_{m \in I}\left|B_{m}\right| \mathbf{1}_{J_{m} \leq j_{n}}\right\} .
$$

The conclusion of the lemma follows by combining equations (S5.21) to (S5.24).

Proof of Lemma 5.2. We use the bound of Lemma 5.1 in conjunction with Theorem 3.1 to obtain a clean bound on $\mathbb{E}_{L} \Pi\left(\mathscr{C}_{*} \cap \mathscr{S}_{I} \mid \mathbf{X}_{n}\right)$. We remark that Lemma 5.1 and Theorem 3.1 imply that $\mathbb{E}_{L} \Pi\left(\mathscr{C}_{*} \cap \mathscr{S}_{I} \mid \mathbf{X}_{n}\right)=0$ whenever $I \cap\left\{m: J_{m}>\log (n) / \log (2)\right\} \neq \varnothing$. Hence, the bound in the lemma holds trivially when $I \cap\left\{m: J_{m}>\log (n) / \log (2)\right\} \neq \varnothing$, and we will now assume without loss of generality that $I \cap\left\{m: J_{m}>\log (n) / \log (2)\right\}=\varnothing$. We will distinguish between two cases, according to whether $\frac{\left(1+\mu_{\star}\right) \log (2)}{2} \sum_{m \in I} J_{m} \mathbf{1}_{J_{m}>j_{n}} \leq \frac{c_{2} n \mathcal{E}_{I}^{2}}{4}$ or not.

Case $\frac{\left(1+\mu_{\star}\right) \log (2)}{2} \sum_{m \in I} J_{m} \mathbf{1}_{J_{m}>j_{n}} \leq \frac{c_{2} n \mathcal{E}_{I}^{2}}{4}$. We claim that $n \mathcal{E}_{I}^{2} \gtrsim \log (n)$ in this scenario. Indeed, if $\sum_{m \in I} \mathbf{1}_{J_{m}>j_{n}} \geq 1$ then $n \mathcal{E}_{I}^{2} \gtrsim \sum_{m \in I} J_{m} \mathbf{1}_{J_{m}>j_{n}} \geq j_{n} \gtrsim \log (n)$ by definition of $j_{n}$. But if $\sum_{m \in I} \mathbf{1}_{J_{m}>j_{n}}=0$, then it must be the case that $\sum_{m \in I} \mathbf{1}_{J_{m} \leq j_{n}} \geq 1$ since by assumption $I \neq \varnothing$. Therefore, $n \mathcal{E}_{I}^{2} \gtrsim \log (n)$ by the estimate of Proposition 7.2. Then, by picking $\alpha=1 / 2$ in Theorem 3.1,

$$
\mathbb{E}_{L} \Pi\left(\mathscr{C}_{*} \cap \mathscr{S}_{I} \mid \mathbf{X}_{n}\right)^{1+2 \delta} \lesssim \exp \left\{-\frac{c_{2} n \mathcal{E}_{I}^{2}}{2}+\frac{c_{1} 2^{J_{0}}|I|}{2}\right\} \frac{\Pi\left(\mathcal{A}_{I}\right)^{1 / 2}}{\Pi\left(\tilde{\mathcal{A}}_{I}\right)^{1 / 2}}
$$

Using the estimate of Lemma 5.1 and the Proposition 7.2, we find that

$$
\begin{aligned}
\frac{1}{2} \log \frac{\Pi\left(\mathcal{A}_{I}\right)}{\Pi\left(\tilde{\mathcal{A}}_{I}\right)} & \leq \frac{c_{4} \log (n)}{2} \sum_{m \in I} 2^{J_{0} \mathbf{1}_{m=0}} \mathbf{1}_{J_{m} \leq j_{n}}-\frac{\left(1+\mu_{\star}\right) \log (2)}{2} \sum_{m \in I} J_{m} \mathbf{1}_{J_{m}>j_{n}} \\
& \leq \frac{c_{4}}{2 C \Gamma^{2}}\left(\xi^{2 \mathbf{1}_{0 \in I}}+2^{J_{0}} \mathbf{1}_{0 \in I}\right) n \mathcal{E}_{I}^{2}-\frac{\left(1+\mu_{\star}\right) \log (2)}{2} \sum_{m \in I} J_{m} \mathbf{1}_{J_{m}>j_{n}} \\
& \leq\left\{\frac{c_{4}\left(\xi^{\left.2 \mathbf{1}_{0 \in I}+2^{J_{0}} \mathbf{1}_{0 \in I}\right)}\right.}{2 C \Gamma^{2}}+\frac{c_{2}}{4}\right\} n \mathcal{E}_{I}^{2}-\left(1+\mu_{\star}\right) \log (2) \sum_{m \in I} J_{m} \mathbf{1}_{J_{m}>j_{n}} .
\end{aligned}
$$

Also, by Proposition 7.2 again,

$$
\begin{aligned}
c_{1} 2^{J_{0}}|I| & =c_{1} 2^{J_{0}} \sum_{m \in I} \mathbf{1}_{J_{m} \leq j_{n}}+c_{1} 2^{J_{0}} \sum_{m \in I} \mathbf{1}_{J_{m}>j_{n}} \\
& \leq \frac{c_{1} 2^{J_{0}}}{C \Gamma^{2} \xi^{-2 \mathbf{1}_{0 \in I} \log (n)}} n \mathcal{E}_{I}^{2}+\frac{c_{1} 2^{J_{0}}}{j_{n}} \sum_{m \in I} J_{m} \mathbf{1}_{J_{m}>j_{n}}
\end{aligned}
$$

So if $\delta>0$ is taken small enough and the constants $K_{1}$ and $K_{4}$ in Assumption 1 are taken large enough, we obtain the bound in the statement of the lemma by combining equations (S5.25) to (S5.27), and because $j_{n} \gtrsim \log (n)$ can be made as small as we want in front of $2^{J_{0}}$ under Assumption 1. 
Case $\frac{\left(1+\mu_{\star}\right) \log (2)}{2} \sum_{m \in I} J_{m} \mathbf{1}_{J_{m}>j_{n}}>\frac{c_{2} n \mathcal{E}_{I}^{2}}{4}$. In this case, by taking $\alpha=\frac{1024 \cdot c_{0} \delta}{1+512 \cdot c_{0} \delta}$ in Theorem 3.1, we obtain that

$$
\begin{aligned}
\mathbb{E}_{L} \Pi\left(\mathscr{C}_{*} \cap \mathscr{S}_{I} \mid \mathbf{X}_{n}\right)^{1+2 \delta} & \leq \frac{3 \exp \left(-\alpha c_{2} n \mathcal{E}_{I}^{2}+\alpha c_{1} 2^{J_{0}}|I|\right)}{\left(1-e^{-c_{2} n \mathcal{E}_{I}^{2}}\right)^{2 \alpha}}\left\{\frac{\Pi\left(\mathcal{A}_{I}\right)}{\Pi\left(\tilde{\mathcal{A}}_{I}\right)}\right\}^{1-\alpha} \\
& \leq \frac{3 \exp \left(\alpha c_{2} n \mathcal{E}_{I}^{2}+\alpha c_{1} 2^{J_{0}}|I|\right)}{\left(c_{2} n \mathcal{E}_{I}^{2}\right)^{2 \alpha}}\left\{\frac{\Pi\left(\mathcal{A}_{I}\right)}{\Pi\left(\tilde{\mathcal{A}}_{I}\right)}\right\}^{1-\alpha}
\end{aligned}
$$

where the second line follows using that $1-e^{-x} \geq x e^{-x}$ for $x \geq 0$. Using the estimate of Lemma 5.1 and the Proposition 7.2

$$
\begin{aligned}
\log \frac{\Pi\left(\mathcal{A}_{I}\right)}{\Pi\left(\tilde{\mathcal{A}}_{I}\right)} & \leq c_{4} \log (n) \sum_{m \in I} 2^{J_{0} \mathbf{1}_{m=0}} \mathbf{1}_{J_{m} \leq j_{n}}-\left(1+\mu_{\star}\right) \log (2) \sum_{m \in I} J_{m} \mathbf{1}_{J_{m}>j_{n}} \\
& \leq \frac{c_{4}\left(\xi^{\left.2 \mathbf{1}_{0 \in I}+2^{J_{0}} \mathbf{1}_{0 \in I}\right)}\right.}{C \Gamma^{2}} n \mathcal{E}_{I}^{2}-\left(1+\mu_{\star}\right) \log (2) \sum_{m \in I} J_{m} \mathbf{1}_{J_{m}>j_{n}} .
\end{aligned}
$$

Hence, for any $t \in(0,1)$, we have,

$$
\begin{aligned}
& \log \frac{\Pi\left(\mathcal{A}_{I}\right)}{\Pi\left(\tilde{\mathcal{A}}_{I}\right)} \leq\left\{-\frac{(1-t) c_{2}}{2}+\frac{c_{4}\left(\xi^{2 \mathbf{1}_{0 \in I}}+2^{J_{0}} \mathbf{1}_{0 \in I}\right)}{C \Gamma^{2}}\right\} n \mathcal{E}_{I}^{2} \\
& -t\left(1+\mu_{\star}\right) \log (2) \sum_{m \in I} J_{m} \mathbf{1}_{J_{m}>j_{n}} .
\end{aligned}
$$

We also note that for this case to happen, it must be that $\left\{m: J_{m}>j_{n}\right\} \neq \varnothing$. Since we have assumed that $I \cap\left\{m: J_{m}>\log (n) / \log (2)\right\}=\varnothing$, by Proposition 7.2

$$
n \mathcal{E}_{I}^{2} \geq C n \gamma^{2} \sum_{m \in I} 2^{-J_{m}(2 s+1)} \mathbf{1}_{J_{m}>j_{n}} \geq C \gamma^{2} n^{-2 s} .
$$

We deduce from the previous that,

$$
\begin{aligned}
\frac{1}{\left(c_{2} n \mathcal{E}_{I}^{2}\right)^{2 \alpha}} & \leq \frac{1}{\left(C c_{2} \gamma^{2}\right)^{2 \alpha}} \exp \{4 \alpha s \log (n)\} \\
& \leq \frac{1}{\left(C c_{2} \gamma^{2}\right)^{2 \alpha}} \exp \left\{4 \alpha s \frac{\log (n)}{j_{n}} \sum_{m \in I} J_{m} \mathbf{1}_{J_{m}>j_{n}}\right\} .
\end{aligned}
$$

But $j_{n} \gtrsim \log (\gamma / \Gamma)+\log (n)$ by equation (3.2), which implies that $j_{n} \gtrsim \log (n)$ when $K_{1}$ in Assumption 1 is taken sufficiently large. So taking in addition $K_{3}$ sufficiently large, we find that there is a universal constant $C^{\prime}>0$ such that

$$
\frac{1}{\left(c_{2} n \mathcal{E}_{I}^{2}\right)^{2 \alpha}} \leq \exp \left\{C^{\prime} \delta \log (2) \sum_{m \in I} J_{m} \mathbf{1}_{J_{m}>j_{n}}\right\} .
$$

So if $\delta>0$ is taken small enough, and the constants $K_{1}, K_{3}$ and $K_{4}$ in Assumption 1 are taken large enough, we obtain the bound in the statement of the lemma by combining equations (S5.27) to (S5.30) and by taking $t=\frac{(1+2 \delta)\left(1+\mu_{\star} / 2+C^{\prime} \delta\right)}{1+\mu_{\star}}$. 


\section{Acknowledgements}

This work was supported by U.S. Air Force Office of Scientific Research grant \#FA9550-15-1-0074. The author also thanks Daniel M. Roy for helpful discussions and the opportunity to work on this project.

\section{References}

CAI, T. T. (2008). On information pooling, adaptability and superefficiency in nonparametric function estimation. Journal of Multivariate Analysis 99421.

CAstillo, I. (2014). On Bayesian supremum norm contraction rates. Ann. Statist. 42 2058-2091.

Ghosal, S., Ghosh, J. K. and VAn Der VAart, A. W. (2000). Convergence rates of posterior distributions. Ann. Statist. 28 500-531.

GHOSAL, S. and VAN DER VAART, A. (2007). Posterior convergence rates of Dirichlet mixtures at smooth densities. Ann. Statist. 35 697-723.

GINÉ, E. and NICKL, R. (2016). Mathematical foundations of infinite-dimensional statistical models 40. Cambridge University Press.

Hoffmann, M., Rousseau, J. and Schmidt-Hieber, J. (2015). On adaptive posterior concentration rates. Ann. Statist. 43 2259-2295.

RivoiraRd, V. and RousseaU, J. (2012). Bernstein-von Mises theorem for linear functionals of the density. Ann. Statist. 40 1489-1523.

VAN DER VAART, A. W. and VAN ZANTEN, J. H. (2008). Rates of contraction of posterior distributions based on Gaussian process priors. Ann. Statist. 36 1435-1463. 